Preprints of the

Max Planck Institute for

Research on Collective Goods

Bonn 2009/27

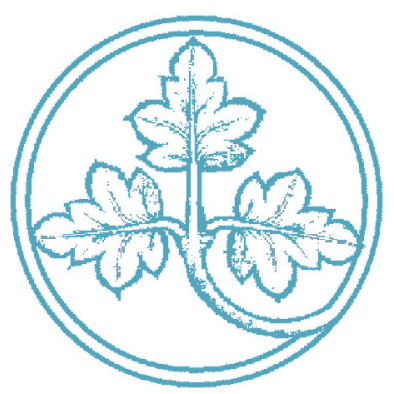

Prices versus Quantities in Electricity Generation

Mark Hahmeier

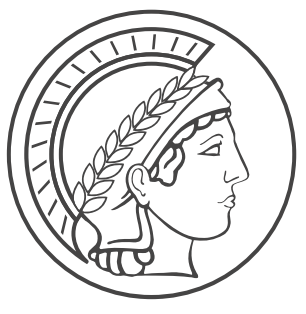




\title{
Prices versus Quantities in Electricity Generation
}

\author{
Mark Hahmeier
}

August 2009 


\title{
Prices versus Quantities in Electricity Generation*
}

\author{
Mark Hahmeier \\ Max Planck Institute for Research on Collective Goods \\ Kurt-Schumacher-Str. 10 \\ D-53113 Bonn, Germany \\ hahmeier@coll.mpg.de \\ August 12, 2009
}

\begin{abstract}
This paper examines the prices versus quantities issue, originally raised by Weitzman [8], in the context of carbon dioxide emissions and with a special focus on electricity generation. Within a simplified model of the electricity market, in which we explicitly allow for a monopolistic gas supplier, we employ a game-theoretic approach and ask, from a welfare perspective, for the superior regulatory regime in response to an expected exercise of market power. Our analysis studies the optimal regulation in each regime and shows that, in the presence of market power in the gas market, taxes rather than permits are the regulator's welfare-maximizing regime choice.
\end{abstract}

JEL Classification: H23, L51, L94, Q54, Q58

Keywords: Climate change, regulation, taxes, emission permits, market power, gas, welfare

\section{Introduction}

A standard method to correct for an externality, as, for example, air pollution and climate change resulting from the burning of coal, is to impose a (linear) tax at the rate of marginal external damages on the use of the entity responsible for the externality. In an idealized deterministic environment with perfect information and no transaction costs, any outcome of a price regulation via (linear) taxes can also be achieved by a regulation based on quantities, e.g., via a cap-and-trade scheme. Under a cap-and-trade scheme, the regulator issues a fixed quantity of permits, each of which allows its holder to generate one predetermined unit of the externality, and allows to trade these allowances, resulting in a market price for permits. In particular, both taxes and the market mechanism for tradeable permits are economically efficient since they allow the externality to be reduced at least cost, i.e., where it is cheapest for the economy. ${ }^{1}$

The European Emissions Trading Scheme (ETS), introduced by the European Union in 2005 in order to reduce carbon dioxide emissions, is one example of a cap-and-trade scheme. Basically, by assigning emission rights and then allowing to trade these rights, the ETS is a restriction on the annual flow of carbon dioxide emissions, covering different industry sectors as, among others, electricity generation, the largest covered sector in terms of emissions. Alternatively, governments could establish a price for carbon by imposing a (linear) tax on emissions. In this paper we compare (linear) taxes and tradeable permits in the context of carbon dioxide emis-

${ }^{*}$ I am especially grateful to Martin Hellwig for offering valuable advice on an earlier version of this paper. Many thanks to Christoph Engel and my colleagues Jos Jansen, Stefan Magen, and Philipp Weinschenk for helpful comments.

${ }^{1}$ Note that either the tax rate or the equilibrium price of permits will equal each originator's marginal costs of reducing the externality. 
sions from electricity generation, although in a specific setting. ${ }^{2}$

Weitzman [8] shows in a seminal paper that, in the presence of uncertainty or asymmetric information about abatement costs and/or environmental damages, a quantity control is preferable to a price control (a linear tax) if and only if the marginal benefit of abatement curve is steeper than the marginal cost of abatement curve and vice versa. ${ }^{3}$ If in the context of climate change, as part of the related literature appears to suggest, marginal costs of abatement are steeper than marginal benefits from reducing a pollutant, then this would be an argument for the use of carbon taxes rather than permits. ${ }^{4}$

In a recent article, Newbery [6] investigates the effect of the European ETS on market power in the gas market and provides another argument for taxes rather than permits. Building on the observation that there is "existing market power in the gas market", ${ }^{5}$ he argues that "fixing the quantity rather than the price of carbon reduces the price elasticity of demand for gas appreciably, amplifying the market power of gas suppliers, and amplifying the impact of gas price increases on the electricity price." According to Newbery, "the amplification of market power works through the impact on the price of EU Emission Allowances, which, as gas prices rise and lead to [the more-carbon intensive fuel] coal substituting for [the less-carbon intensive fuel] gas, raise emissions and hence the price of EU Emission Allowances, which in turn favours gas, offsetting the normal market demand response to an increase in the price of gas."

Similar to Newbery [6], it is the aim of our paper to compare (linear) taxes and tradeable permits in the context of carbon dioxide emissions from electricity generation, explicitly taking into account the effects of market power in the gas market. In contrast to Newbery, however, we employ a game-theoretic approach and study the optimal regulation in response to an expected exercise of market power: In each regime, taxes and permits, we search for that level of the regulatory device which is welfare-optimal given that the gas supplier in turn and from his perspective optimally responds in exercising market power, and compare the two in order to find the regulator's welfare-superior regime choice. In order to elicit the effects of market power in

\footnotetext{
${ }^{2}$ For a general discussion, cf. Grubb and Newbery [1]; or K. Neuhoff [5]. For a recent review of the literature on prices versus quantities, cf. Hepburn [2].

${ }^{3}$ Intuitively, if marginal costs are approximately constant and marginal benefits are steep, then the smallest deviation from the tax level corresponding to the optimal quantity (which equates marginal benefits and marginal costs from abatement) results (in the quantity at which marginal costs equal the tax rate and thus) in a high deviation from the optimal quantity - specifying a quantity directly is the better alternative. On the other hand, if marginal benefits are approximately constant and marginal costs are steep, even a high deviation in the tax level will only result in a small deviation from the optimal quantity — using a tax and letting the originator(s) choose a quantity close to the optimal level is the better alternative. Note that many subsequent contributions have modified the assumptions in Weitzman's uncertainty analysis. One strand of literature, for example, discusses the prices versus quantities question in a dynamic context where environmental damages are caused by a pollution stock rather than being a (quadratic) function of the flow of pollution (cf., for example, Newell and Pizer [7]).

${ }^{4}$ Cf. Neuhoff [5], Section 3.2; or Newbery [6], Section 5: "For most pollutants the marginal abatement cost schedule is fairly flat and low for modest abatement, but rises rapidly as a higher fraction of emissions is to be curtailed. The damage contributed by emissions today is effectively the same as those tomorrow, and so the marginal benefit of abatement is essentially flat at each moment, while the marginal cost of abatement rises rapidly beyond a certain point, arguing for taxes rather than quotas."

${ }^{5}$ In the words of Newbery [6], Section 2.3: "While the international market for coal is reasonably competitive, the same is not true for gas, particularly in Europe, which is heavily dependent on importing Russian gas from the monopoly supplier, Gazprom. In addition, gas producers and suppliers in the EU have more market power than the suppliers of other fuels." Cf. Grubb and Newbery [1], Section 4.1.
} 
the gas market, we deviate from Weitzman's analysis in that we presume perfect information (about abatement costs and environmental damages).

In the following, we develop a simplified model of the electricity market (with elastic electricity demand), in which we explicitly introduce a gas supplier endowed with market power. Such exercise of market power has a direct effect on electricity producers, who, in our model, can reduce emissions (and react to relative price changes between coal, gas and permits or taxes) merely by substituting coal-based by gas-based production. The regulator, taking into account the gas supplier's strategic response to her policy choice, aims at maximizing welfare from electricity generation. Our notion of welfare excludes the gas supplier's profits resulting from his monopolistic behaviour; ${ }^{6}$ our analysis, however, outlines the respective consequences of welfare maximization for the gas supplier's profits.

In order to examine the effects of market power in the gas market, our game-theoretic analysis contrasts two different scenarios. In the "competitive scenario", the gas price is set at marginal costs. In the "monopolistic scenario", the gas supplier chooses his price in order to maximize profits. Based on the only presumption that coal-based per-unit-of-electricity emissions are above gas-based per-unit-of-electricity emissions, ${ }^{7}$ our analysis comes to the following conclusions (which refer to Proposition 1 to 5 in our analysis, respectively):

1) In the competitive scenario, where the gas price equals marginal gas production costs, we obtain the expected benchmark result: The regulator's welfare-maximizing policy in each regime implies the same welfare level. Taxes and permits are equivalent.

2) In the emission taxes regime, the regulator's welfare-maximizing tax level choice equals the marginal welfare costs of emissions, in both the competitive and the monopolistic scenario. In other words, market power in the gas market has no effect on the optimal tax level; and, the higher the welfare costs of emissions, the higher is the optimal tax level.

3) In the emission permits regime, market power in the gas market has an effect on the optimal permits level, and the direction of this effect depends on the magnitude of the difference between coal-based and gas-based per-unit-of-electricity emissions: The regulator's welfaremaximizing permits level choice in the monopolistic scenario is higher than his optimal choice in the competitive scenario if and only if the difference between coal-based and gas-based per-unit-of-electricity emissions is sufficiently high. ${ }^{8}$

4) In the monopolistic scenario, i.e., whenever there is market power in the gas market, taxes are the regulator's welfare-maximizing regime choice, except for very specific parameter constellations, under which taxes and permits guarantee for the same welfare and result in the same profits for the gas supplier. And, except for these specific parameter constellations,

5) maximizing welfare in the monopolistic scenario of the emission taxes regime implies higher profits for the gas supplier than maximizing welfare in the permits regime.

\footnotetext{
${ }^{6}$ Referring also to the preceding footnote, the reader can think of the European case and the Russian gas supplier Gazprom (which does not belong to the European Union and could be considered as external in terms of welfare considerations).

${ }^{7}$ Cf. Neuhoff [5], Section 1.2: "For example in the electricity sector, burning natural gas instead of coal for power generation can reduce carbon emissions by about $50 \%$ per unit of electricity produced."

${ }^{8}$ Note that in the monopolistic scenario, as we will explain, the regulator's optimal binding (i.e., effectively restricting the electricity producers) permits level is welfare-superior to a non-binding permits level if and only if the marginal welfare costs of emissions are sufficiently high. Our result concerning the regulator's welfaremaximizing permits level choice refers to this case, i.e., to the optimal binding permits level.
} 
We proceed as follows. Section 2 introduces our simple model. Section 3 outlines the benchmark result obtained for the competitive scenario. Section 4 and 5 are devoted to the analysis of the monopolistic taxes and permits regime, respectively. Section 6 compares the two regimes. A mathematical presentation of all (intermediate) results and corresponding proofs can be found in Appendix B. Remarks and standard calculations are deferred to Appendix A and C, respectively.

\section{Our Model}

Our simplified model of the electricity market consists of an environmental regulator, a gas supplier, and electricity producers. Electricity consumers enter only through their demand $E(\cdot)$, which is a function of electricity price $\omega \in \mathbf{R}_{++}$, and is derived from their maximization of utility from electricity consumption $U(\cdot)$, a function of units $E \in \mathbf{R}_{+}$consumed, minus corresponding $\operatorname{costs} \omega \cdot E$ via $U^{\prime}(E)=\omega .^{9}$

For the supply of electricity, each producer can make use of two independent technologies, one based on the input coal, the other based on the input gas. Electricity producer $i$ 's corresponding production function is denoted by $F^{(i)}(K, G)=F_{\text {coal }}^{(i)}(K)+F_{\text {gas }}^{(i)}(G)$, where $K \in \mathbf{R}_{+}$and $G \in \mathbf{R}_{+}$denote the input units of coal and gas, respectively. An electricity producer is assumed to maximize profits by choosing the input quantities appropriately, taking as given gas price $\gamma \in \mathbf{R}_{++}$, coal price $p_{K} \in \mathbf{R}_{++}$, the equilibrium price of electricity $\omega^{*}$, and, depending on the regime, either tax level ("carbon price") $p \in \mathbf{R}_{+}$or the equilibrium price of emission permits $P^{*}$.

Assuming that total emissions from the production of electricity by producer $i$ can be expressed as a function $e^{(i)}(\cdot, \cdot)$ of the input units $K_{i}$ and $G_{i}$ used, electricity producer $i$ 's profit is given by $\Pi^{(i)}\left(K_{i}, G_{i}\right)=\omega \cdot F^{(i)}\left(K_{i}, G_{i}\right)-p_{K} \cdot K_{i}-\gamma \cdot G_{i}-x \cdot e^{(i)}\left(K_{i}, G_{i}\right)$, where $x$ either equals tax level $p$ (in an emission taxes regime) or the price of permits $P$ (in an emission permits regime). In an emission taxes regime, letting $\left(K_{i}^{*}(\omega, p), G_{i}^{*}(\omega, p)\right)$ denote electricity producer $i$ 's profit-maximizing input combination as a function of electricity price $\omega$ and tax level $p$, the equilibrium price of electricity $\omega^{*}$ equates demand and supply in the market for electricity and is thus determined by $E\left(\omega^{*}\right)=\sum_{i} F^{(i)}\left(K_{i}^{*}\left(\omega^{*}, p\right), G_{i}^{*}\left(\omega^{*}, p\right)\right)$. In an emission permits regime, letting $\left(K_{i}^{*}(\omega, P), G_{i}^{*}(\omega, P)\right)$ denote electricity producer $i$ 's profit-maximizing input combination as a function of electricity price $\omega$ and permits price $P$, equilibrium prices $\omega^{*}$ and $P^{*}$ are determined by $E\left(\omega^{*}\right)=\sum_{i} F^{(i)}\left(K_{i}^{*}\left(\omega^{*}, P^{*}\right), G_{i}^{*}\left(\omega^{*}, P^{*}\right)\right)$ and $\sum_{i} e^{(i)}\left(K_{i}^{*}\left(\omega^{*}, P^{*}\right), G_{i}^{*}\left(\omega^{*}, P^{*}\right)\right)=a$, where $a \in \mathbf{R}_{+}$denotes the level of emission allowances.

In our analysis we abstract from the allocation process (which is presumed to be efficient) within the supply side of the electricity market, and assume that both production and emission functions are the same across (non-strategic and price-taking) producers. ${ }^{10}$ This allows us to model the supply side of the electricity market by introducing one (representative) electricity producer, whose choice maximizes

$$
\Pi^{(\text {permits })}(K, G):=\omega \cdot F(K, G)-p_{K} \cdot K-\gamma \cdot G \text { subject to } e(K, G) \leq a
$$

\footnotetext{
${ }^{9}$ Note that all functions are specified in more detail later in this section.

${ }^{10}$ For literature on the theory of environmental regulation in imperfectly competitive markets, cf., for example, Mansur [4]. In particular, Mansur examines the welfare implications of prices versus quantities when firms have market power in selling a polluting good (esp. electricity) and the environmental regulator assumes a competitive product market. For a recent article which reviews the literature on market power in pollution permit markets, cf. Montero [3].
} 
in an emission permits regime, ${ }^{11}$ and

$$
\Pi^{(\text {taxes })}(K, G):=\omega \cdot F(K, G)-p_{K} \cdot K-\gamma \cdot G-p \cdot e(K, G)
$$

in an emission taxes regime, taking as given the price of gas and coal, and the equilibrium price of electricity determined by

$$
E(\omega)=F(K(\omega), G(\omega)) .
$$

In our model, the gas supplier can supply gas at per-unit costs of $\psi \in \mathbf{R}_{++}$. Whereas coal price $p_{K}$ is assumed to be set on competitive markets, we consider two different scenarios with respect to the gas supplier's price choice: In the competitive scenario, gas price $\gamma$ equals marginal/average cost $\psi$; in the monopolistic scenario, the gas supplier chooses $\gamma \in[\psi, \infty)$ so as to maximize profits.

The regulator aims at maximizing welfare from electricity generation. If the coal suppliers generate zero economic profit, then a measure of welfare, excluding the gas supplier's profits, can be defined by the difference between the utility from electricity consumption and the sum of the factor costs of electricity production and the social costs of emissions:

$$
W=U(E)-p_{K} \cdot K-\gamma \cdot G-q \cdot e(K, G),
$$

where "marginal emission cost" $q \in \mathbf{R}_{++}$is a measure of the per-unit-of-emissions welfare. The regulator's choice is the regime and the level of the regulatory device, i.e., the tax or permits level. In summary, the sequence of actions in the monopolistic scenario is as illustrated in Figure 1 .

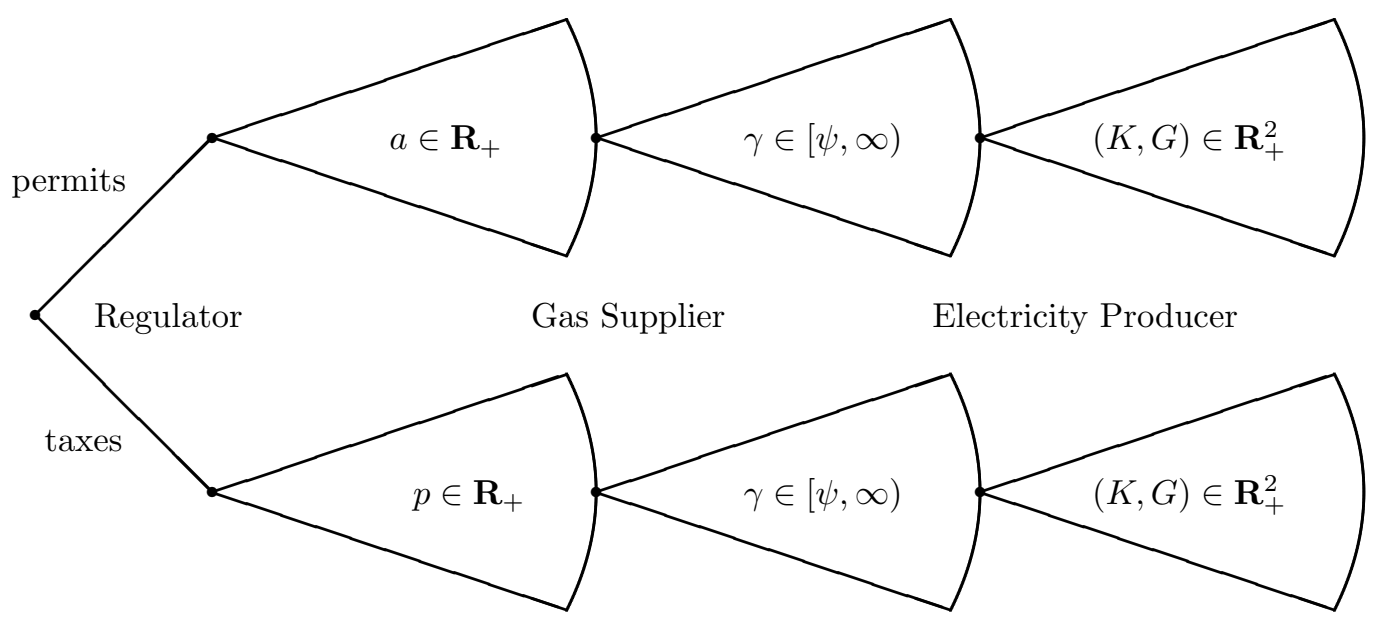

Figure 1

Our analysis of the two regimes follows standard backward induction and concentrates on the following specifications. Electricity demand is assumed to be linear in price, i.e.,

$$
E(\omega)=\beta_{0}-\beta_{1} \cdot \omega
$$

for some constants $\beta_{0}, \beta_{1} \in \mathbf{R}_{++}$. The electricity producer's production function satisfies

$$
F(K, G)=F_{\text {coal }}(K)+F_{\text {gas }}(G)=2 \cdot f_{K} \cdot \sqrt{K}+2 \cdot f_{G} \cdot \sqrt{G}
$$

for some constants $f_{K}, f_{G} \in \mathbf{R}_{++}$. And, emissions from the production of electricity depend on

\footnotetext{
${ }^{11}$ Essentially, the price equilibrium in the market for permits reduces to a quantity constraint for the electricity producer. A positive equilibrium permits price corresponds to a binding quantity constraint. Cf. Appendix A.
} 
the respective input used, and are linear in the respective units of electricity produced:

$$
e(K, G)=2 \cdot \delta_{K} \cdot \sqrt{K}+2 \cdot \delta_{G} \cdot \sqrt{G}
$$

for some constants $\delta_{G}, \delta_{K} \in \mathbf{R}_{++}$. In other words, we assume that there exist two constants $\tilde{\delta}_{K}$, $\tilde{\delta}_{G} \in \mathbf{R}_{++}$(representing coal-based and gas-based per-unit-of-electricity emissions, respectively) such that $e(K, G)=\tilde{\delta}_{K} \cdot F_{\text {coal }}(K)+\tilde{\delta}_{G} \cdot F_{\text {gas }}(G)=\tilde{\delta}_{K} \cdot 2 f_{K} \sqrt{K}+\tilde{\delta}_{G} \cdot 2 f_{G} \sqrt{G}$, and define $\delta_{K}:=\tilde{\delta}_{K} f_{K}$ and $\delta_{G}:=\tilde{\delta}_{G} f_{G}$. If electricity demand is determined by $U^{\prime}(E)=\omega=\frac{\beta_{0}-E}{\beta_{1}}$, then

$$
U(E)=\frac{\beta_{0}}{\beta_{1}} \cdot E-\frac{1}{2 \beta_{1}} \cdot E^{2} .
$$

Our analysis concentrates on the interior input factor area of electricity production and abstracts from the possibility that electricity could in principle be produced by using either gas or coal but not both. Technically, we restrict the electricity producer's choice set to the open area $(K, G) \in \mathbf{R}_{++}^{2}$ and require each player's equilibrium strategy to specify an action only (more precisely: exactly) at those of her/his decision nodes where there exists at least one action that results (via sequential rationality) in a well-defined outcome of the game and at the same time maximizes the player's target (welfare or profit) over all alternatives with a well-defined outcome; each player's equilibrium strategy must specify such an action at each of these decision nodes. ${ }^{12}$ In order to guarantee the existence of an equilibrium in each regime, ${ }^{13}$ we assume that marginal emission cost $q$ satisfies

$$
q<\bar{q}:=\frac{\beta_{0} f_{K} \psi}{R},{ }^{14}
$$

where $R$ and $D$ are as defined below. Furthermore, we presume that coal-based per-unit-ofelectricity emissions $\tilde{\delta}_{K}$ are higher than gas-based per-unit-of-electricity emissions $\tilde{\delta}_{G}$. Since $f_{K} \delta_{G}-f_{G} \delta_{K}=f_{K} \tilde{\delta}_{G} f_{G}-f_{G} \tilde{\delta}_{K} f_{K}=f_{K} f_{G}\left(\tilde{\delta}_{G}-\tilde{\delta}_{K}\right)$, this is equivalent to the requirement that

$$
D:=f_{K} \delta_{G}-f_{G} \delta_{K}<0 .
$$

The results of our analysis depend, in part, on whether this measure of the difference between coal-based and gas-based per-unit-of-electricity emissions is (relatively) high or low, more precisely on whether

$$
Q:=\beta_{1} \delta_{G} p_{K}+2 f_{K} D
$$

is lower or greater than (or equal to) zero. For notational convenience, we define (on $\gamma \in \mathbf{R}_{++}$)

$$
\begin{gathered}
R(\gamma):=\beta_{1} \delta_{K} \gamma-2 f_{G} D, \\
\Psi(\gamma):=\beta_{1}\left(\delta_{G}^{2} p_{K}+\delta_{K}^{2} \gamma\right)+2 D^{2}, \text { and } \\
\Phi(\gamma):=\left(\beta_{1} p_{K}+2 f_{K}^{2}\right) \gamma+2 f_{G}^{2} p_{K},
\end{gathered}
$$

and abbreviate $R:=R(\psi), \Psi:=\Psi(\psi)$, and $\Phi:=\Phi(\psi)$.

\footnotetext{
${ }^{12}$ In other words, we require the electricity producer's equilibrium strategy to specify an action only at those of his decision nodes where previous decisions (about the gas price and the tax or permits level) allow him to maximize profits by choosing an input factor combination which is strictly positive in each component. Correspondingly, we require the gas supplier's equilibrium strategy to specify a gas price choice only at those of his decision nodes where the tax or permits level allows him to maximize profits over all alternatives which allow the electricity producer to maximize his profits within $(K, G) \in \mathbf{R}_{++}^{2}$. And, finally, the regulator's equilibrium (regime and tax or permits level) choice must maximize welfare over all alternatives that allow the gas supplier to maximize profits over all alternatives which allow the electricity producer to maximize his profits within $(K, G) \in \mathbf{R}_{++}^{2}$.

${ }^{13}$ Note that, in the following, the word 'equilibrium' often refers to the subgame starting after the regulator's regime choice.

${ }^{14}$ Cf. Appendix B, Lemma 1c, part f) of both Lemma 2.1 and 2.2, and Lemma 3a and b.
} 
Finally, note that, in principle, an emission permits level can be either binding or not. Whether or not a permits level will be binding, i.e., effectively restricting the electricity producer's choice, depends on both the regulator's preceding permits level choice and the gas supplier's price response. Given a specific permits level choice, it might well be in the gas supplier's interest to respond with a gas price resulting in a non-binding permits level. And, taking into account the gas supplier's strategic response to her policy choice, it is a priori not clear whether the regulator would prefer a binding to a non-binding permits level from a welfare perspective. Therefore, our analysis has to keep track of both cases. ${ }^{15}$ Before we proceed, please remember that a mathematical derivation of all the following is provided in Appendix B.

\section{Analysis of the Competitive Scenario}

In the competitive scenario, gas price $\gamma$ equals marginal/average cost $\psi$. Correspondingly, our four-stage game formally reduces to three non-trivial stages: First, the regulator chooses the regime and level of the regulatory device. Afterwards, the electricity producer's choice of the two input quantities determines the combination of coal- and gas-based production.

In any equilibrium of the emission taxes regime, the electricity producer's profit maximization, taking as given gas price $(\gamma=) \psi$ and coal price $p_{K}$, together with market clearing in the market for electricity implies that factor inputs $K$ and $G$ equal

$$
K^{*}:=\left(\frac{\beta_{0} \gamma f_{K}-p R(\gamma)}{\Phi(\gamma)}\right)^{2}=\left(\frac{\beta_{0} \psi f_{K}-p R}{\Phi}\right)^{2} \text { and } G^{*}:=\left(\frac{\beta_{0} p_{K} f_{G}-p Q}{\Phi(\gamma)}\right)^{2}=\left(\frac{\beta_{0} p_{K} f_{G}-p Q}{\Phi}\right)^{2},
$$

respectively. Given these levels of coal- and gas-based electricity production, welfare, as a function of tax level $p$, can be calculated as (a quadratic function of $p$ )

$$
W_{\psi}(p):=\frac{-2 \beta_{1} \Psi \cdot p^{2}+4 \beta_{1} q \Psi \cdot p+2 \beta_{0}\left[\beta_{0}\left(f_{K}^{2} \psi+f_{G}^{2} p_{K}\right)-2 \beta_{1} q\left(f_{K} \delta_{K} \psi+\delta_{G} f_{G} p_{K}\right)\right]}{2 \beta_{1} \Phi} .
$$

It follows that the regulator's welfare-maximizing tax level choice is $p^{*}:=q$, i.e., the optimal tax level equals the marginal welfare costs of emissions, resulting in welfare level

$$
W_{\psi}\left(p^{*}\right)=W^{C}(q):=\frac{\beta_{1} \Psi \cdot q^{2}-2 \beta_{1} \beta_{0}\left(f_{K} \delta_{K} \psi+f_{G} \delta_{G} p_{K}\right) \cdot q+\beta_{0}^{2}\left(f_{K}^{2} \psi+f_{G}^{2} p_{K}\right)}{\beta_{1} \Phi} .
$$

Lemma 1 in Appendix B confirms that $\left(K^{*}\left(p^{*}\right), G^{*}\left(p^{*}\right), p^{*}\right)$ is the unique equilibrium in the competitive scenario of the emission taxes regime.

In the emission permits regime, equilibrium factor inputs depend upon whether or not the permits level is binding, i.e., upon whether or not the permits level effectively restricts the electricity producer's profit maximization, taking as given gas price $\psi$, coal price $p_{K}$, and the market clearing price of electricity. In any equilibrium of the emission permits regime, factor inputs $K$ and $G$ equal

$$
K_{A}^{*}:=\left(\frac{\beta_{0} \gamma f_{K}}{\Phi(\gamma)}\right)^{2}=\left(\frac{\beta_{0} \psi f_{K}}{\Phi}\right)^{2} \text { and } G_{A}^{*}:=\left(\frac{\beta_{0} p_{K} f_{G}}{\Phi(\gamma)}\right)^{2}=\left(\frac{\beta_{0} p_{K} f_{G}}{\Phi}\right)^{2}
$$

respectively, if the permits level is non-binding, and they equal

and

$$
K_{B}^{*}:=\left(\frac{2 \beta_{0} \delta_{G} D+a R(\gamma)}{2 \Psi(\gamma)}\right)^{2}=\left(\frac{2 \beta_{0} \delta_{G} D+a R}{2 \Psi}\right)^{2}
$$

$$
G_{B}^{*}:=\left(\frac{2 \beta_{0} \delta_{K}(-D)+a Q}{2 \Psi(\gamma)}\right)^{2}=\left(\frac{2 \beta_{0} \delta_{K}(-D)+a Q}{2 \Psi}\right)^{2}
$$

respectively, if the emission permits level is binding. In particular, an emission permits level is

\footnotetext{
${ }^{15}$ In particular, as it turns out, the regulator's decision for or against a binding permits level depends on whether or not marginal emission cost $q$ is greater or lower than$$
\tilde{q}(Q) \stackrel{\text { def }}{=}\left\{\begin{array}{ll}
\frac{\beta_{0} p_{K} f_{G}(-Q)}{2 \beta_{1} \delta_{K}^{2} \Phi} & \text { if } Q \leq 0 \\
\frac{\beta_{0} p_{K} f_{G} Q}{2\left(\beta_{1} p_{K}+2 f_{K}^{2}\right) \Psi} & \text { if } Q \geq 0
\end{array} .\right.
$$ 
binding if and only if it is lower than $e\left(K_{A}^{*}, G_{A}^{*}\right)=2 \delta_{K}\left(\frac{\beta_{0} \psi f_{K}}{\Phi}\right)+2 \delta_{G}\left(\frac{\beta_{0} p_{K} f_{G}}{\Phi}\right)=: a_{1}$. In the latter case, welfare, as a function of permits level $a$, can be calculated as

$$
W_{\psi}(a):=\frac{-\beta_{1} \Phi \cdot a^{2}+\left[4 \beta_{0} \beta_{1}\left(f_{K} \delta_{K} \psi+f_{G} \delta_{G} p_{K}\right)-4 \beta_{1} q \Psi\right] \cdot a+4 \beta_{0}^{2} D^{2}}{4 \beta_{1} \Psi} .
$$

It follows that the regulator's welfare-maximizing permits level choice, if going for the binding permits level alternative, is $a_{C}^{*}(q):=a_{1}-\frac{2 \Psi}{\Phi} q>0$, resulting in welfare level

$$
W_{\psi}\left(a_{C}^{*}\right)=W_{B}^{C}(q):=\frac{1}{\beta_{1} \Psi \Phi}\left[\beta_{1}\left(\beta_{0}\left(f_{K} \delta_{K} \psi+f_{G} \delta_{G} p_{K}\right)-q \Psi\right)^{2}+\beta_{0}^{2} D^{2} \Phi\right] .
$$

If, on the other hand, the emission permits level is (chosen such that it is) non-binding, then the resulting welfare equals that of the taxes regime (i.e., $\left.W_{\psi}(p)\right)$ for tax level $p=0$, which is lower than $W_{B}^{C}(q)$. In other words, the regulator's welfare-maximizing permits level choice is $a_{C}^{*}$. Lemma 2 in Appendix B confirms that $\left(K_{B}^{*}\left(a_{C}^{*}\right), G_{B}^{*}\left(a_{C}^{*}\right), a_{C}^{*}\right)$ is the unique equilibrium in the competitive scenario of the emission permits regime, and one with a binding permits level. Comparing the welfare levels of the two equilibria, we obtain the expected benchmark result.

\section{Proposition 1}

In the competitive scenario, where gas price $\gamma$ equals the gas supplier's per-unit cost $\psi$, the gas supplier's profits are zero, and the regulator's optimal policy in each regime (tax level $q$ in the taxes regime and permits level $a_{C}^{*}$ in the permits regime) implies the same welfare level: $W_{B}^{C}(q)=W^{C}(q)$ (cf. Lemma $3 c$ in Appendix B). In particular, both taxes and permits can be part of an equilibrium of the three-stage game starting with the regulator's regime choice.

\section{Analysis of the Emission Taxes Regime}

In the monopolistic scenario of the emission taxes regime, in contrast to the competitive scenario, the gas supplier can optimally react in response to the regulator's tax level choice, given his perception about the electricity producer's corresponding choice of the two input quantities. In any equilibrium of the emission taxes regime, the electricity producer's profit maximization, taking as given gas price $\gamma$ and coal price $p_{K}$, together with market clearing in the market for electricity implies that factor inputs $K$ and $G$ equal ( $K^{*}$ and $G^{*}$ as defined in Section 3, i.e.,)

$$
K^{*} \stackrel{\text { def }}{=}\left(\frac{\beta_{0} \gamma f_{K}-p R(\gamma)}{\Phi(\gamma)}\right)^{2} \text { and } G^{*} \stackrel{\text { def }}{=}\left(\frac{\beta_{0} p_{K} f_{G}-p Q}{\Phi(\gamma)}\right)^{2}
$$

respectively. Given these levels of coal- and gas-based electricity production, welfare, as a function of tax level $p$, can be calculated as

$$
W_{\gamma}(p):=\frac{\tilde{W}_{2}(\gamma) \cdot p^{2}+\tilde{W}_{1}(\gamma) \cdot p+\tilde{W}_{0}(\gamma)}{2 \beta_{1} \Phi(\gamma)},
$$

where $\tilde{W}_{2}(\gamma):=-2 \beta_{1} \Psi(\gamma)<0, \tilde{W}_{1}(\gamma):=4 \beta_{1} q \Psi(\gamma)>0$, and $\tilde{W}_{0}(\gamma):=2 \beta_{0}\left[\beta_{0}\left(f_{K}^{2} \gamma+f_{G}^{2} p_{K}\right)-\right.$ $\left.2 \beta_{1} q\left(f_{K} \delta_{K} \gamma+\delta_{G} f_{G} p_{K}\right)\right]$. In particular, if gas price $\gamma$ does not depend on tax level $p$, then welfare $W_{\gamma}(p)$ is maximized at $p=q$, independent of the specific $\gamma$.

Since the gas supplier's profits, given by $(\gamma-\psi) \cdot G^{*}(\gamma)$, are maximized at

$$
\gamma^{*}:=\frac{\Phi}{\beta_{1} p_{K}+2 f_{K}^{2}}+\psi,
$$

which is independent of the regulator's tax level choice $p,{ }^{16}$ the regulator's welfare-maximizing tax level choice is $p^{*}:=q$, i.e., the optimal tax level equals the marginal costs of emissions.

\footnotetext{
${ }^{16}$ This independence of the optimal gas price from the regulator's tax level choice results, in particular, from the property that gas demand $G^{*}$ and thus the gas supplier's profit as a function of gas price $\gamma$ and tax level $p$ is separable in these two parameters, i.e., can be written as the product of two functions, one in $\gamma$, the other in $p$.
} 
Note that two ingredients of our model are responsible for the regulator's welfare-maximizing tax level choice to equal marginal emission cost $q$ in both the competitive and monopolistic scenario: First, the gas supplier's optimal price choice in the monopolistic scenario is independent of the regulator's tax level choice, as is $\psi$ in the competitive scenario; and, second, welfare at a specific gas price is maximized by choosing the tax level equal to $q$, independent of the specific (value of the) gas price.

To illustrate the second property, consider welfare $W_{\gamma}(p)$ as composed of utility and costs. The sum of factor and emission costs, a quadratic function of tax level $p$, is decreasing in $p$ (as $G^{*}$, $K^{*}$, and electricity demand $E^{*}=F\left(K^{*}, G^{*}\right)$ are decreasing in $p$ ). For the same reason, also the utility from electricity consumption, which again is a quadratic function of tax level $p$, is decreasing in $p$. The optimal tax level maximizes the difference between the two, i.e., between utility and costs. In other words, at $q$ the slopes of the two curves with respect to tax level $p$ are the same:

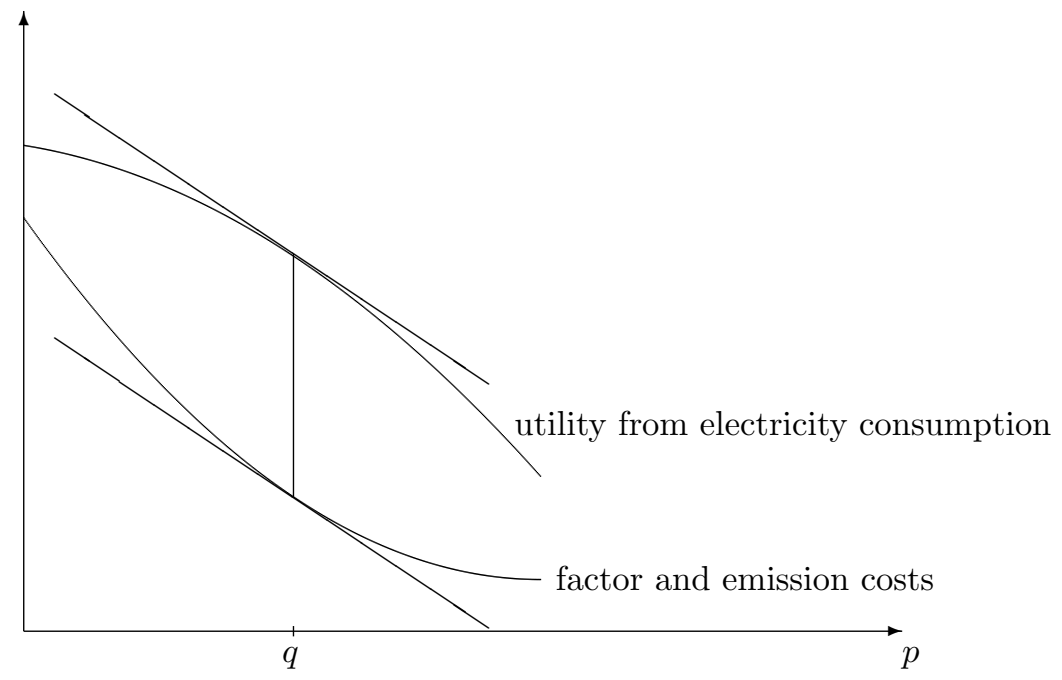

Figure 2

Considering now the effect of a change in gas price $\gamma$, we observe the following. The slope of the utility curve with respect to tax level $p$ and at each specific $p$ is decreasing in the gas price: The utility effect of an increase in gas price $\gamma$ on the optimal tax level is negative. However, as outlined above, this effect is exactly neutralized by a corresponding change in (the slope of) factor and emission costs. Tax level $q$ remains the point where the slopes of the two curves with respect to tax level $p$ are the same.

If the regulator chooses tax level $p^{*}=q$ and the gas supplier gas price $\gamma^{*}$, then welfare equals

$$
W_{\gamma^{*}}\left(p^{*}\right)=W^{M}(q):=\frac{\tilde{W}_{2}^{M} \cdot q^{2}+\tilde{W}_{1}^{M} \cdot q+\tilde{W}_{0}^{M}}{2 \beta_{1} \Phi},
$$

where $\tilde{W}_{0}^{M}:=\beta_{0}^{2}\left(f_{K}^{2} \psi+f_{G}^{2} p_{K}+\frac{f_{K}^{2} \Phi}{\beta_{1} p_{K}+2 f_{K}^{2}}\right), \tilde{W}_{1}^{M}:=-2 \beta_{1} \beta_{0}\left(f_{K} \delta_{K} \psi+f_{G} \delta_{G} p_{K}+\frac{f_{K} \delta_{K} \Phi}{\beta_{1} p_{K}+2 f_{K}^{2}}\right)$, and $\tilde{W}_{2}^{M}:=\beta_{1}\left(\Psi+\frac{\beta_{1} \delta_{K}^{2}}{\beta_{1} p_{K}+2 f_{K}^{2}} \Phi\right)$. The gas supplier's corresponding profits are

$$
\left(\gamma^{*}-\psi\right) \cdot G^{*}\left(\gamma^{*}, p^{*}\right)=\Gamma(q):=\frac{\left(\beta_{0} p_{K} f_{G}-q Q\right)^{2}}{4 \Phi \cdot\left(\beta_{1} p_{K}+2 f_{K}^{2}\right)}>0 .
$$

Lemma 1 in Appendix B confirms that $\left(K^{*}\left(\gamma^{*}, p^{*}\right), G^{*}\left(\gamma^{*}, p^{*}\right), \gamma^{*}, p^{*}\right)$ is the unique equilibrium in the monopolistic scenario of the emission taxes regime. Proposition 2 summarizes our results.

\section{Proposition 2}

In the monopolistic scenario of the emission taxes regime, the regulator's welfare-maximizing tax level choice equals marginal emission cost $q$ as in the competitive scenario, resulting in welfare level $W^{M}(q)$ and profits $\Gamma(q)$ for the gas supplier. 


\section{Analysis of the Emission Permits Regime}

Our analysis of the emission permits regime depends upon whether measure $Q$ of the difference between coal-based and gas-based per-unit-of-electricity emissions is lower than, greater than, or equal to zero. The following three paragraphs are devoted to these three cases, respectively.

\subsection{The Case of a High Difference in Per-Unit-of-Electricity Emissions}

Suppose that $Q<0$ and consider the monopolistic scenario. As already mentioned earlier, in the emission permits regime, equilibrium factor inputs depend upon whether or not the permits level is in fact binding, i.e., upon whether or not the permits level effectively restricts the electricity producer's profit maximization, taking as given gas price $\gamma$, coal price $p_{K}$, and the market clearing price of electricity. In particular, in any equilibrium of the emission permits regime factor inputs $K$ and $G$ equal

$$
K_{A}^{*} \stackrel{\text { def }}{=}\left(\frac{\beta_{0} \gamma f_{K}}{\Phi(\gamma)}\right)^{2} \text { and } G_{A}^{*} \stackrel{\text { def }}{=}\left(\frac{\beta_{0} p_{K} f_{G}}{\Phi(\gamma)}\right)^{2},
$$

respectively, if the emission permits level is non-binding, and they equal

$$
K_{B}^{*} \stackrel{\text { def }}{=}\left(\frac{2 \beta_{0} \delta_{G} D+a R(\gamma)}{2 \Psi(\gamma)}\right)^{2} \text { and } G_{B}^{*} \stackrel{\text { def }}{=}\left(\frac{2 \beta_{0} \delta_{K}(-D)+a Q}{2 \Psi(\gamma)}\right)^{2}
$$

respectively, if the emission permits level is binding.

In case of a binding permits level, the gas supplier's profits are given by $(\gamma-\psi) \cdot G_{B}^{*}(\gamma)$ and maximized at gas price

$$
\gamma_{B}^{*}:=\frac{\Psi}{\beta_{1} \delta_{K}^{2}}+\psi
$$

Defining $C_{B}(a):=\left(\gamma_{B}^{*}-\psi\right) \cdot G_{B}^{*}\left(\gamma_{B}^{*}\right)$, curve $C_{B}(\cdot)$ reflects the gas supplier's achievable profits in case of a binding permits level. It is a quadratic function of $a$, which obtains its minimum value 0 at some level $a_{6}>0$.

In case of a non-binding permits level, the gas supplier's profits are given by $(\gamma-\psi) \cdot G_{A}^{*}(\gamma)$ and maximized at $\gamma_{A}^{*}:=\gamma^{*}$ (cf. Section 4). Defining $C_{A}(a):=\left(\gamma^{*}-\psi\right) \cdot G_{A}^{*}\left(\gamma^{*}\right)$, curve $C_{A}(\cdot)$ reflects the gas supplier's achievable profits in case of a non-binding permits level. It is a constant function of $a$, i.e., $C_{A}(a)$ has the same value $\bar{C}_{A}$ for every permits level $a$.

Denoting the two intersections of $C_{B}(\cdot)$ with $C_{A}(\cdot)$ by $a_{3}<a_{6}$ and $a_{7}>a_{6}$, Figure 3 illustrates the two curves. The precise "values" of variables $a_{0}$ to $a_{7}$ are provided in Appendix B.

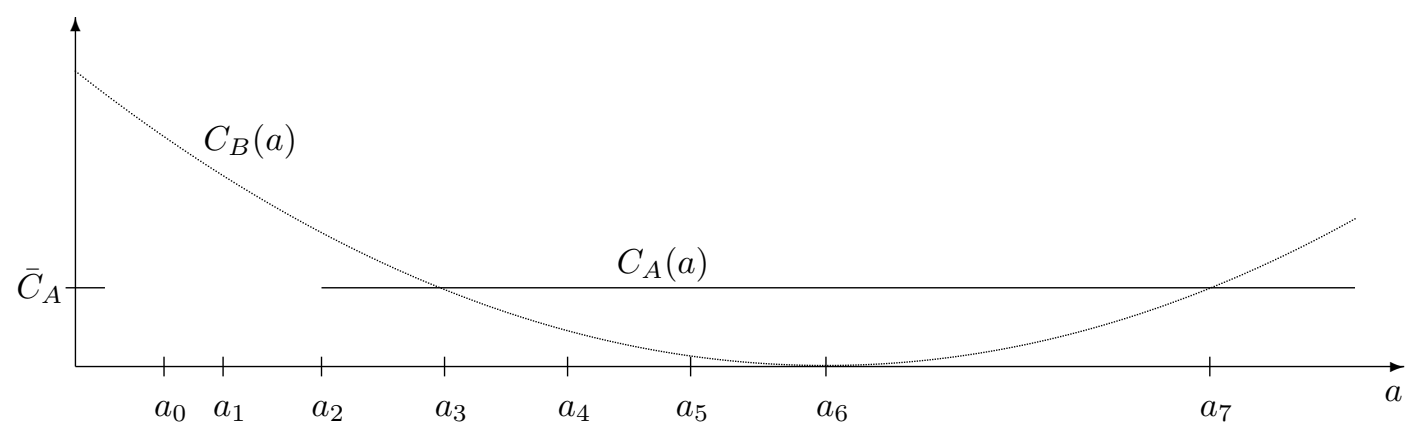

Figure 3

If going for the binding permits level alternative, the gas supplier maximizes his profit $(\gamma-\psi)$. $G_{B}^{*}(\gamma)$ subject to $2 \delta_{K} \sqrt{K_{A}^{*}}+2 \delta_{G} \sqrt{G_{A}^{*}}>a$, i.e., subject to the permits level being binding. Note that the inequality constraint cannot be satisfied for non-negative gas prices if (and only if) permits level $a$ is greater than or equal to some level $a_{5} \in\left(a_{3}, a_{6}\right)$. In other words, going for the binding permits level alternative requires $a<a_{5}$. Furthermore, the inequality constraint allows to choose $\gamma_{B}^{*}$ if and only if permits level $a$ is lower than some level $a_{4} \in\left(a_{3}, a_{5}\right)$. 
If going for the non-binding permits level alternative, the gas supplier maximizes his profit $(\gamma-\psi) \cdot G_{A}^{*}(\gamma)$ subject to $2 \delta_{K} \sqrt{K_{A}^{*}}+2 \delta_{G} \sqrt{G_{A}^{*}} \leq a$, i.e., subject to the permits level being non-binding. The latter inequality constraint allows to choose $\gamma_{A}^{*}$ if and only if permits level $a$ is greater than or equal to some level $a_{2} \in\left(0, a_{3}\right)$.

Therefore, assuming that the gas supplier has a preference for a binding (in comparison to a non-binding) permits level (if indifferent with respect to profits), the gas supplier goes for the binding permits level alternative if and only if $a \leq a_{3}$. In other words, if $a \leq a_{3}$, then gas price $\gamma$ equals $\gamma_{B}^{*}$ and the permits level is binding. If $a>a_{3}$, then gas price $\gamma$ equals $\gamma_{A}^{*}$, and the permits level is non-binding.

In the latter case, the gas supplier's profits are

$$
\Gamma_{A}:=\frac{\left(\gamma_{A}^{*}-\psi\right)\left(\beta_{0} p_{K} f_{G}\right)^{2}}{\Phi\left(\gamma_{A}^{*}\right)^{2}}=\frac{\left(\beta_{0} p_{K} f_{G}\right)^{2}}{4 \Phi \cdot\left(\beta_{1} p_{K}+2 f_{K}^{2}\right)}>0,
$$

and the resulting welfare level, which is independent of $a$ and in the following referred to as $W_{A}^{M}(q)$, equals that of the taxes regime (i.e., $\left.W_{\gamma^{*}}(p)\right)$ for tax level $p=0$, and is thus lower than its counterpart $W^{M}(q)$ in the taxes regime (where tax level $p$ equals $p^{*}=q>0$ ).

Having analyzed the gas supplier's decision, we continue with the regulator's choice of the optimal permits level. If the permits level is binding, then welfare, as a function of permits level $a$, can be calculated as

$$
W_{\gamma}(a):=\frac{\tilde{V}_{2}(\gamma) \cdot a^{2}+\tilde{V}_{1}(\gamma) \cdot a+\tilde{V}_{0}(\gamma)}{4 \beta_{1} \Psi(\gamma)}
$$

where $\tilde{V}_{2}(\gamma):=-\beta_{1} \Phi(\gamma)<0, \tilde{V}_{1}(\gamma):=4 \beta_{0} \beta_{1}\left(f_{K} \delta_{K} \gamma+f_{G} \delta_{G} p_{K}\right)-4 \beta_{1} q \Psi(\gamma)$, and $\tilde{V}_{0}(\gamma):=4 \beta_{0}^{2} D^{2}>0$. It follows that the regulator's welfare-maximizing permits level choice, if going for the binding permits level alternative, is

$$
\hat{a}_{M}^{*}(q):=\min \left\{a_{M}^{*}(q):=\frac{2 \beta_{0}\left(f_{K} \delta_{K} \psi+f_{G} \delta_{G} p_{K}\right)+\frac{2 \beta_{0} f_{K}}{\beta_{1} \delta_{K}} \Psi-4 q \Psi}{\Phi+\left(\beta_{1} p_{K}+2 f_{K}^{2}\right) \frac{\Psi}{\beta_{1} \delta_{K}^{2}}}, a_{3}\right\}>0,
$$

and, in particular, higher than his welfare-maximizing permits level choice $a_{C}^{*}$ in the competitive scenario. We comment on the latter result at the end of this section. The value of emission cost $q$ at which $a_{M}^{*}(q)$ equals $a_{3}$ is positive and in the following referred to as $q_{3}$.

If the regulator chooses permits level $\hat{a}_{M}^{*}$ and the gas supplier gas price $\gamma_{B}^{*}$, and if marginal emission cost $q$ is greater than or equal to $q_{3}$ (and thus $\hat{a}_{M}^{*}=a_{M}^{*}$ ), then welfare equals

$$
\begin{aligned}
& W_{\gamma_{B}^{*}}\left(a_{M}^{*}\right)=W_{B}^{M}(q):=\frac{\beta_{1}\left[\beta_{0}\left(f_{K} \delta_{K} \psi+f_{G} \delta_{G} p_{K}\right)+\frac{\beta_{0} f_{K}}{\beta_{1} \delta_{K}} \Psi-2 q \Psi\right]^{2}+\beta_{0}^{2} D^{2}\left(\Phi+\frac{\beta_{1} p_{K}+2 f_{K}^{2}}{\beta_{1} \delta_{K}^{2}} \Psi\right)}{2 \beta_{1} \Psi \cdot\left(\Phi+\frac{\beta_{1} p_{K}+2 f_{K}^{2}}{\beta_{1} \delta_{K}^{2}} \Psi\right)}, \\
& \text { the gas supplier's profits are }
\end{aligned}
$$

$$
\Gamma_{B}(q):=\left(\gamma_{B}^{*}-\psi\right) \cdot \frac{\left[2 \beta_{0} \delta_{K}(-D)+Q a_{M}^{*}\right]^{2}}{4 \Psi\left(\gamma_{B}^{*}\right)^{2}}=\frac{\beta_{1} \delta_{K}^{2} \Psi \cdot\left(\beta_{0} f_{G} p_{K}-q Q\right)^{2}}{\left[\beta_{1} \delta_{K}^{2} \Phi+\left(\beta_{1} p_{K}+2 f_{K}^{2}\right) \Psi\right]^{2}}>0 .
$$

If $q<q_{3}$ (and thus $\hat{a}_{M}^{*}=a_{3}<a_{M}^{*}$ ), then $W_{B}^{M}(q)$ is an upper boundary for the resulting welfare.

Comparing this welfare level to the one resulting from a non-binding permits level, we obtain

$$
W_{A}^{M}(q)>W_{B}^{M}(q) \Leftrightarrow q \gtrless \tilde{q}(Q) \stackrel{(\text { on }}{:=}<\frac{\beta_{0} p_{K} f_{G}(-Q)}{2 \beta_{1} \delta_{K}^{2} \Phi} .
$$

Thus, the optimal binding emission permits level $\hat{a}_{M}^{*}$ is welfare-superior to any non-binding one (i.e., to any permits level above $a_{3}$ ) if and only if marginal emission cost $q$ exceeds level $\tilde{q}(Q)\left(>q_{3}\right)$ : Only if marginal emission cost $q$ is sufficiently high, then the advantages of restricting the electricity producer's emissions in a binding manner, namely lower emission costs, outweigh the corresponding changes in factor costs and electricity production (and thus in utility from electricity consumption).

Lemma 2 in Appendix B confirms that $\left(K_{B}^{*}\left(\gamma_{B}^{*}, a_{M}^{*}\right), G_{B}^{*}\left(\gamma_{B}^{*}, a_{M}^{*}\right), \gamma_{B}^{*}, a_{M}^{*}\right)$ is the unique equilibrium in the monopolistic scenario of the emission permits regime whenever $Q<0$ and $q>\tilde{q}(Q) .{ }^{17}$

\footnotetext{
${ }^{17}$ And, Lemma 4 confirms that there are parameter constellations allowing $q$ to satisfy $\tilde{q}(Q)<q<\bar{q}$.
} 
Proposition 3.1 summarizes our results.

\section{Proposition 3.1}

Suppose that $Q<0$ and consider the monopolistic scenario of the emission permits regime.

a) If marginal emission cost $q$ is greater than $\tilde{q}(Q)$, then the regulator's welfare-maximizing permits level choice is $a_{M}^{*}$, resulting in welfare level $W_{B}^{M}(q)$ and profits $\Gamma_{B}(q)$ for the gas supplier. The regulator's permits level choice is binding and higher than his choice $a_{C}^{*}$ in the competitive scenario.

b) If $q \leq \tilde{q}(Q)$, then achievable welfare equals $W_{A}^{M}(q)$ (via choosing a non-binding permits level) and is below that of the taxes regime, $W^{M}(q)$. The difference in achievable welfare between the two regimes, $W^{M}(q)-W_{A}^{M}(q)$, increases in marginal emission cost $q$ (cf. Lemma 2.1e in Appendix B) and equals $W^{M}(q)-W_{B}^{M}(q)$ at $q=\tilde{q}(Q)$. The gas supplier's profits are $\Gamma_{A}$.

According to case a) of Proposition 3.1, the regulator's welfare-maximizing binding permits level choice is higher in the monopolistic than in the competitive scenario. In order to understand why this is the case, consider the different components of welfare, which is defined as the difference between utility from electricity consumption and factor plus emission costs. As we have seen, for $(\gamma, a)$ equal to $\left(\psi, a_{C}^{*}\right)$ or $\left(\gamma_{B}^{*}, \hat{a}_{M}^{*}\right)$, factor inputs for coal and gas equal $K_{B}^{*}(a, \gamma)$ and $G_{B}^{*}(a, \gamma)$, respectively, and electricity demand equals $E^{*}(a, \gamma)=F\left(K^{*}(a, \gamma), G^{*}(a, \gamma)\right)=$ $\Psi(\gamma)^{-1}\left[2 \beta_{0} D^{2}+\beta_{1} a\left(f_{K} \delta_{K} \gamma+f_{G} \delta_{G} p_{K}\right)\right]$. If in the monopolistic scenario the permits level were chosen to be $a_{C}^{*}\left(<\hat{a}_{M}^{*}\right)$ instead of $\hat{a}_{M}^{*}$, then the resulting factor inputs for coal and gas would equal $K^{*}\left(a_{C}^{*}, \gamma_{B}^{*}\right)<K^{*}\left(\hat{a}_{M}^{*}, \gamma_{B}^{*}\right)$ and $G^{*}\left(a_{C}^{*}, \gamma_{B}^{*}\right)>G^{*}\left(\hat{a}_{M}^{*}, \gamma_{B}^{*}\right)$, respectively, and electricity demand would equal $E^{*}\left(a_{C}^{*}, \gamma_{B}^{*}\right)<E^{*}\left(\hat{a}_{M}^{*}, \gamma_{B}^{*}\right)$. Therefore, by allowing the electricity producer more emissions in the monopolistic scenario (via choosing $\hat{a}_{M}^{*}$ instead of $a_{C}^{*}$ ), the regulator is responsible for higher emission costs (since permits level $\hat{a}_{M}^{*}$ is binding) and higher factor costs for $\operatorname{coal}\left(p_{K} \cdot K^{*}\left(\hat{a}_{M}^{*}, \gamma_{B}^{*}\right)>p_{K} \cdot K^{*}\left(a_{C}^{*}, \gamma_{B}^{*}\right)\right)$. However, the higher emission permits level implies, at the same time, a higher electricity demand and thus a higher utility from electricity consumption as well as lower factor costs for gas $\left(\gamma_{B}^{*} \cdot G^{*}\left(\hat{a}_{M}^{*}, \gamma_{B}^{*}\right)<\gamma_{B}^{*} \cdot G^{*}\left(a_{C}^{*}, \gamma_{B}^{*}\right)\right)$. As Proposition 3.1 states, this second effect overcompensates the higher costs with respect to emissions and coal.

\subsection{The Case of a Low Difference in Per-Unit-of-Electricity Emissions}

Suppose now that $Q>0$, and note that equilibrium factor inputs (as functions of gas price $\gamma$ and permits level $a)$ do not change: Factor inputs $(K, G)$ equal $\left(K_{A}^{*}, G_{A}^{*}\right)$ in the non-binding and $\left(K_{B}^{*}, G_{B}^{*}\right)$ in the binding case. The role of a higher/lower permits level $a$ in gas demand $G_{B}^{*}$, however, reverses. In particular, curve $C_{B}(\cdot)$, which reflects the gas supplier's achievable profits in case of a binding permits level, is now increasing in and positive on $a \geq 0$, and also $a_{2}>a_{4}>a_{5}>0$. Figure 4 illustrates the two curves $C_{B}(\cdot)$ and $C_{A}(\cdot)$ in case of $Q>0$.

If going for the non-binding permits level alternative, the gas supplier maximizes his profit $(\gamma-\psi) \cdot G_{A}^{*}(\gamma)$ subject to $2 \delta_{K} \sqrt{K_{A}^{*}}+2 \delta_{G} \sqrt{G_{A}^{*}} \leq a$. The latter inequality constraint allows for a non-negative gas price $\gamma$ if and only if $a>a_{5}$. And, if $a>a_{5}$, then the inequality constraint is equivalent to

$$
\gamma \geq \hat{\gamma}(a):=\frac{a \cdot 2 f_{G}^{2} p_{K}-2 \beta_{0} f_{G} \delta_{G} p_{K}}{2 \beta_{0} \delta_{K} f_{K}-a \cdot\left(\beta_{1} p_{K}+2 f_{K}^{2}\right)},
$$

which allows to choose gas price $\gamma_{A}^{*}$ if and only if permits level $a$ is greater than or equal to $a_{2}\left(\hat{\gamma}\left(a_{2}\right)=\gamma_{A}^{*}\right)$, resulting in profit $\bar{C}_{A}$ for the gas supplier. If $a \in\left(a_{5}, a_{2}\right)$, then the gas supplier's profit is maximized by choosing $\hat{\gamma}(a)$, and his corresponding profit is $\hat{\Gamma}_{A}(a):=$ $(\hat{\gamma}(a)-\psi) \cdot G_{A}^{*}(\hat{\gamma}(a))>0 . \hat{\Gamma}_{A}(\cdot)$ is a concave quadratic function of $a$, which obtains its maximum $\bar{C}_{A}$ at $a_{2}$ and equals $C_{B}\left(a_{4}\right)$ at $a_{4}$. 


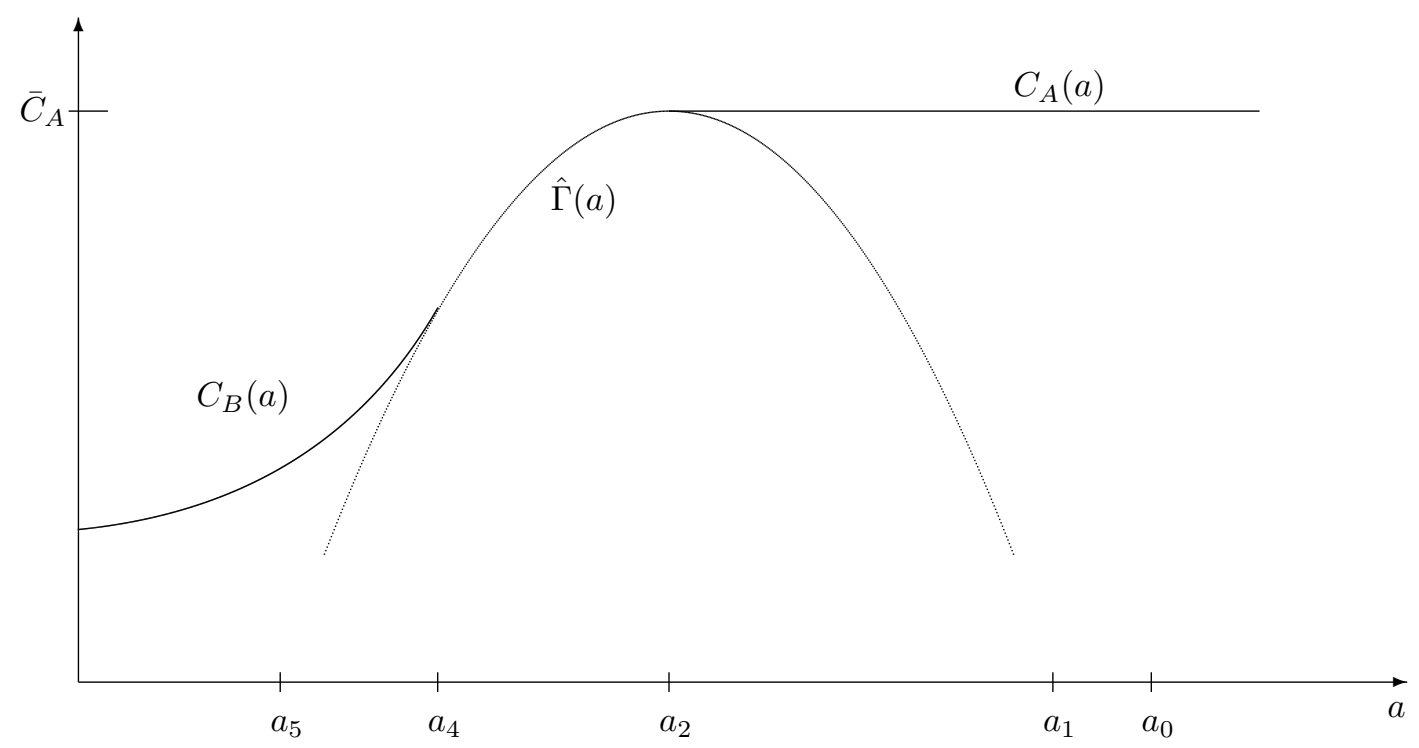

Figure 4

If going for the binding permits level alternative, the gas supplier maximizes $(\gamma-\psi) \cdot G_{B}^{*}(\gamma)$ subject to $2 \delta_{K} \sqrt{K_{A}^{*}}+2 \delta_{G} \sqrt{G_{A}^{*}}>a$, i.e., subject to the permits level being binding. The inequality constraint allows for a non-negative gas price $\gamma$ if and only if permits level $a$ is lower than some level $a_{0}>a_{2}$. And, if $a<a_{0}$, then the inequality constraint is equivalent to $\gamma<\hat{\gamma}(a)$, which allows to choose gas price $\gamma_{B}^{*}$ if and only if $a$ is lower than $a_{4}\left(\hat{\gamma}\left(a_{4}\right)=\gamma_{B}^{*}\right)$, resulting in profit $C_{B}(a)$ for the gas supplier. If $a \in\left[a_{4}, a_{0}\right)$, then the gas supplier's achievable profit is lower than $(\hat{\gamma}(a)-\psi) \cdot G_{B}^{*}(\hat{\gamma}(a))$, which equals $\hat{\Gamma}_{A}(a)$.

Since $\hat{\Gamma}_{A}(a)$ is below $C_{B}(a)$ on $a<a_{4}$ and below $\bar{C}_{A}$ on $a>a_{2}$, it follows that the gas supplier goes for the binding permits level alternative if and only if $a<a_{4}$. In other words, if $a<a_{4}$, then gas price $\gamma$ equals $\gamma_{B}^{*}$ and the permits level is binding. If $a \in\left[a_{4}, a_{2}\right]$ or $a \geq a_{2}$, then gas price $\gamma$ equals $\hat{\gamma}(a)$ or $\gamma_{A}^{*}$, respectively, and the permits level is non-binding.

Having analyzed the gas supplier's decision, we continue with the regulator's choice of the optimal permits level.

If the emission permits level is non-binding and $a \geq a_{2}$, then the gas supplier's profits are $\Gamma_{A}>0$ and the resulting welfare level, which is independent of $a$, equals that of the taxes regime for tax level $p=0$ and thus $W_{A}^{M}(q)$. In particular, it is lower than its counterpart $W^{M}(q)$ in the taxes regime (where tax level $p$ equals $p^{*}=q>0$ ).

If the emission permits level is non-binding and $a \in\left[a_{4}, a_{2}\right]$, then welfare, as a function of permits level $a$, can be calculated as

$$
\hat{W}^{M}(a):=\frac{\tilde{W}_{0}(\hat{\gamma}(a))}{2 \beta_{1} \Phi(\hat{\gamma}(a))}=\frac{2 \beta_{0}^{2} f_{K} D+\beta_{1}\left(\beta_{0} f_{G} p_{K}-2 Q q\right) \cdot a}{2 \beta_{1} Q} .
$$

It follows that, whenever $\beta_{0} f_{G} p_{K}-2 Q q<0$, the regulator's welfare-maximizing permits level choice, if going for the non-binding permits level alternative, is $a_{4}$. The resulting welfare level $\hat{W}^{M}\left(a_{4}\right)$ is in the following denoted by $\hat{W}_{A}^{M}(q)$. If, on the other hand, $q$ is lower than or equal to $\frac{\beta_{0} f_{G} p_{K}}{2 Q}$, then any $a \geq a_{2}$ or any $a \geq a_{4}$, respectively, maximizes welfare, resulting in welfare level $W_{A}^{M}(q)\left(=\hat{W}^{M}\left(a_{2}\right)\right)$.

If the emission permits level is binding, then welfare, as a function of permits level $a$, equals $W_{\gamma}(a)$, and the regulator's welfare-maximizing permits level choice, if going for the binding permits level alternative, is

$$
a_{M}^{*}(q) \stackrel{\text { def }}{=} a_{4}-4 \Psi \cdot\left(\Phi+\frac{\beta_{1} p_{K}+2 f_{K}^{2}}{\beta_{1} \delta_{K}^{2}} \Psi\right)^{-1}\left(<a_{4}\right),
$$

resulting in welfare level $W_{B}^{M}(q)$ and profits $\Gamma_{B}(q)$ for the gas supplier. Since $Q>0$, now the regulator's permits level choice is lower than his choice $a_{C}^{*}$ in the competitive scenario. 
Comparing the different welfare levels, we obtain $\hat{W}_{A}^{M}(q)<W_{B}^{M}(q)$ and

$$
W_{A}^{M}(q)>W_{B}^{M}(q) \Leftrightarrow q \gtrless \tilde{q}(Q) \stackrel{(\text { on }}{:=>0)} \frac{\beta_{0} p_{K} f_{G} Q}{2\left(\beta_{1} p_{K}+2 f_{K}^{2}\right) \Psi} .
$$

Thus, if marginal emission cost $q$ exceeds level $\tilde{q}(Q)$, then the regulator's welfare-maximizing permits level choice is $a_{M}^{*}$, the emission permits level is binding, and the resulting welfare level equals $W_{B}^{M}(q)$. If $q<\tilde{q}(Q)$, which implies that $q<\frac{\beta_{0} f_{G} p_{K}}{2 Q}$, then any permits level $a \geq a_{2}$ maximizes welfare, is non-binding, and results in welfare level $W_{A}^{M}(q)$. If $q=\tilde{q}(Q)$, which also implies that $q<\frac{\beta_{0} f_{G} p_{K}}{2 Q}$, then welfare is maximized by choosing either $a_{M}^{*}$ (resulting in a binding permits level) or any level $a \geq a_{2}$ (resulting in a non-binding permits level), and the resulting welfare equals $W_{B}^{M}(q)=W_{A}^{M}(q)$.

Lemma 2 in Appendix B confirms that $\left(K_{B}^{*}\left(\gamma_{B}^{*}, a_{M}^{*}\right), G_{B}^{*}\left(\gamma_{B}^{*}, a_{M}^{*}\right), \gamma_{B}^{*}, a_{M}^{*}\right)$ is the unique equilibrium in the monopolistic scenario of the emission permits regime whenever $Q>0$ and $q>\tilde{q}(Q){ }^{18}$ Proposition 3.2 summarizes our results.

\section{Proposition 3.2}

Suppose $Q>0$ and consider the monopolistic scenario of the emission permits regime.

a) If marginal emission cost $q$ is greater than $\tilde{q}(Q)$, then the regulator's welfare-maximizing permits level choice is $a_{M}^{*}$, resulting in welfare level $W_{B}^{M}(q)$ and profits $\Gamma_{B}(q)$ for the gas supplier. The regulator's permits level choice is binding and lower than his choice $a_{C}^{*}$ in the competitive scenario.

b) If $q \leq \tilde{q}(Q)$, then achievable welfare equals $W_{A}^{M}(q)$ and is below that of the taxes regime, $W^{M}(q)$. The difference in achievable welfare between the two regimes, $W^{M}(q)-W_{A}^{M}(q)$, increases in $q$ (cf. Lemma 2.2e in Appendix B) and equals $W^{M}(q)-W_{B}^{M}(q)$ at $q=\tilde{q}(Q)$. The gas supplier's profits equal $\Gamma_{A}$.

\subsection{The Borderline Case $(Q=0)$}

Suppose that $Q=0$, and note that equilibrium factor inputs (as functions of $\gamma$ and $a$ ) again equal $\left(K_{A}^{*}, G_{A}^{*}\right)$ in the non-binding and $\left(K_{B}^{*}, G_{B}^{*}\right)$ in the binding case. Since $Q=0$, gas demand $G_{B}^{*}$ is now independent of the regulator's permits level choice, curve $C_{B}(\cdot)$ is constant in $a$ and equals $\bar{C}_{A}$, and $a_{0}=a_{1}=a_{2}=a_{4}=a_{5}$.

If going for the binding permits level alternative, the gas supplier maximizes $(\gamma-\psi) \cdot G_{B}^{*}(\gamma)$ subject to $2 \delta_{K} \sqrt{K_{A}^{*}}+2 \delta_{G} \sqrt{G_{A}^{*}}>a$. The inequality constraint allows for a non-negative gas price $\gamma$ if and only if $a<a_{5}$, and allows to choose gas price $\gamma_{B}^{*}$ if and only if $a<a_{4}\left(=a_{5}\right)$, resulting in profit $\bar{C}_{A}$ for the gas supplier.

If going for the non-binding permits level alternative, the gas supplier maximizes $(\gamma-\psi) \cdot G_{A}^{*}(\gamma)$ subject to $2 \delta_{K} \sqrt{K_{A}^{*}}+2 \delta_{G} \sqrt{G_{A}^{*}} \leq a$. The latter inequality constraint is satisfied for all $\gamma \geq 0$ if $a \geq a_{5}$, and allows for a positive gas price $\gamma$ if and only if $a \geq a_{5}$.

It follows that the gas supplier goes for the binding alternative (via choosing $\gamma_{B}^{*}$ ) if $a<a_{5}$, and for the non-binding alternative (via choosing $\gamma_{A}^{*}$ ) if $a \geq a_{5}$. His profit is $\Gamma_{A}=\bar{C}_{A}$ in either case.

As before, if the emission permits level is non-binding, then the resulting welfare level equals that of the taxes regime for tax level $p=0$ and thus $W_{A}^{M}(q)$. If the permits level is binding, then welfare, as a function of permits level $a$, equals $W_{\gamma}(a)$, and the regulator's welfare-maximizing permits level choice, if going for the binding alternative, is $a_{M}^{*}(q)$, resulting in welfare level $W_{B}^{M}(q)$.

\footnotetext{
${ }^{18}$ And, Lemma 4 confirms that there are parameter constellations allowing $q$ to satisfy $\tilde{q}(Q)<q<\bar{q}$.
} 
Since $Q=0, a_{M}^{*}(q)$ equals his choice $a_{C}^{*}$ in the competitive scenario, and $W_{B}^{M}(q)>W_{A}^{M}(q)$ for all $q>\tilde{q}(0):=0$. Lemma 2 in Appendix B confirms that $\left(K_{B}^{*}\left(\gamma_{B}^{*}, a_{M}^{*}\right), G_{B}^{*}\left(\gamma_{B}^{*}, a_{M}^{*}\right), \gamma_{B}^{*}, a_{M}^{*}\right)$ is the unique equilibrium in the monopolistic scenario of the emission permits regime if $Q=0$.

\section{Proposition 3.3}

If, in the monopolistic scenario of the emission permits regime, $Q=0$, then the regulator's welfare-maximizing permits level choice is $a_{M}^{*}$, resulting in welfare level $W_{B}^{M}(q)$ and profits $\Gamma_{B}(q)=\Gamma_{A}$ for the gas supplier. The regulator's permits level choice is binding and equals his choice $a_{C}^{*}$ in the competitive scenario.

\section{Permits versus Taxes}

From Proposition $3.1 \mathrm{~b}$ and $3.2 \mathrm{~b}$ we already know that in the monopolistic scenario taxes are welfare-superior whenever marginal emission cost $q$ is sufficiently low: If $q \leq \tilde{q}(Q)$ (which implies $Q \neq 0$ ), then any non-binding permits level choice provides for at least the same welfare as the optimal binding permits level, and for less welfare than the optimal tax level. The following result, Proposition 4, which is based on Lemma 3a in Appendix B, implies that taxes are welfare-maximizing for all $q$. In particular, Proposition 4 compares the resulting welfare in case of an optimal binding permits level (cf. Proposition 3.1a, 3.2a and 3.3) with the welfare level achievable in an emission taxes regime, and shows that the gas supplier's ability to optimally respond to the regulator's choice makes it impossible for a binding permits level to result in a higher welfare level than the regulator's optimal tax choice.

\section{Proposition 4 (Welfare in the Monopolistic Scenario)}

Suppose that, in the monopolistic scenario, marginal emission cost $q$ is greater than $\tilde{q}(Q)$. Then the difference in welfare between the unique equilibrium of the taxes regime and the unique (and binding permits level) equilibrium of the permits regime, namely $W^{M}(q)-W_{B}^{M}(q)$, is nonnegative; the difference is greater than zero if and only if $Q \neq 0$, it is increasing in marginal emission cost $q$ if $Q<0$ and decreasing in $q$ if $Q>0$.

Combining our findings from Proposition $3.1 \mathrm{~b}$ and $3.2 \mathrm{~b}$ for the case $q \leq \tilde{q}(Q)$ and from Proposition 4 covering marginal emission costs greater than $\tilde{q}(Q)$, we obtain the following result.

Corollary Whenever $Q \neq 0,\left(K^{*}\left(\gamma^{*}, p^{*}\right), G^{*}\left(\gamma^{*}, p^{*}\right), \gamma^{*}, p^{*}\right.$,"taxes") is the unique equilibrium of the four-stage game starting with the regulator's regime choice. Only if $Q=0$, then both $\left(K^{*}\left(\gamma^{*}, p^{*}\right), G^{*}\left(\gamma^{*}, p^{*}\right), \gamma^{*}, p^{*}, "\right.$ taxes" $)$ and $\left(K_{B}^{*}\left(\gamma_{B}^{*}, a_{M}^{*}\right), G_{B}^{*}\left(\gamma_{B}^{*}, a_{M}^{*}\right), \gamma_{B}^{*}, a_{M}^{*}\right.$, "permits") constitute an equilibrium of the four-stage game starting with the regulator's regime choice.

In particular, if the difference between coal-based and gas-based per-unit-of-electricity emissions is high such that $Q<0$, then the difference in achievable welfare between the two regimes increases in the marginal welfare costs of emissions: Taxes are welfare-superior and the higher marginal emission cost $q$, the greater is this superiority. If the difference between coal-based and gas-based per-unit-of-electricity emissions is low such that $Q>0$, then taxes are welfare-superior and the difference in achievable welfare between the two regimes increases in low and decreases in high values of $q$.

We close our analysis and comparison of the two regimes with an evaluation of the gas supplier's profits in Proposition 5, which is based on Lemma 3b in Appendix B. In particular, Proposition 5 compares the respective profits for the gas supplier outlined in Proposition 3.1 to 3.3 across 
all possible equilibria of the two regimes. The subsequent corollary highlights the main result.

\section{Proposition 5 (The Gas Supplier's Profits in the Monopolistic Scenario)}

If, in the monopolistic scenario, $q \geq \tilde{q}(Q)$, then the difference in the resulting gas supplier's profits between the unique equilibrium of the taxes regime and the binding permits level equilibrium, $\left(K_{B}^{*}\left(\gamma_{B}^{*}, a_{M}^{*}\right), G_{B}^{*}\left(\gamma_{B}^{*}, a_{M}^{*}\right), \gamma_{B}^{*}, a_{M}^{*}\right)$, namely $\Gamma(q)-\Gamma_{B}(q)$, is non-negative; it is greater than zero if and only if $Q \neq 0$. If $q \leq \tilde{q}(Q)$ (which implies $Q \neq 0$ ), then the difference in the resulting gas supplier's profits between the unique equilibrium of the taxes regime and any non-binding permits level equilibrium of the permits regime, namely $\Gamma(q)-\Gamma_{A}$, is greater than zero.

Corollary In the monopolistic scenario, whenever $Q \neq 0$, then the gas supplier's profits in the unique equilibrium of the emission taxes regime are higher than his profits would be in any equilibrium of the emission permits regime. Only if $Q=0$, then his profits in the unique equilibrium of the emission taxes regime and the unique (and binding permits level) equilibrium of the permits regime are the same.

Summarizing our final results about the monopolistic scenario, taxes are the regulator's welfaremaximizing regime choice whenever $Q \neq 0$; in addition, maximizing welfare in the emission taxes regime implies higher profits for the gas supplier than maximizing welfare in the emission permits regime. And even if the difference between coal-based and gas-based per-unit-of-electricity emissions is such that $Q=0$, taxes guarantee for the same welfare and result in the same profits for the gas supplier as permits.

\section{A Remarks}

In order to see that, as suggested in Section 2, the price equilibrium in the market for permits reduces to a quantity constraint and that a positive equilibrium permits price corresponds to a binding quantity constraint, suppose that, without loss of generality, there is only one electricity producer (instead of considering several identical producers), whose choice of factor inputs $(K, G) \in \mathbf{R}_{++}$maximizes $\Pi(K, G):=\omega \cdot F(K, G)-p_{K} \cdot K-\gamma \cdot G-P \cdot e(K, G)$, taking as given the equilibrium price for electricity and for permits. Then, the electricity producer's corresponding profit-maximizing input combination $(K(\omega, P), G(\omega, P))$ satisfies $\frac{\partial \Pi}{\partial K}=\frac{\partial \Pi}{\partial G}=0$, which implies $\sqrt{K(\omega, P)}=\frac{\omega f_{K}-\delta_{K} P}{p_{K}}$ and $\sqrt{G(\omega, P)}=\frac{\omega f_{G}-\delta_{G} P}{\gamma}$. Market clearing in the market for electricity requires $E(\omega)=\beta_{0}-\beta_{1} \omega=2 f_{K} \sqrt{K(\omega, P)}+2 f_{G} \sqrt{G(\omega, P)}=F(K(\omega, P), G(\omega, P))$, i.e., $\omega=\omega^{*}(P):=\frac{\beta_{0} p_{K} \gamma+2 f_{K} \delta_{K} P \gamma+2 f_{G} \delta_{G} p_{K} P}{\Phi(\gamma)}$. Defining

$$
K^{*}(P):=K\left(\omega^{*}(P), P\right)=\left(\frac{\beta_{0} \gamma f_{K}-P R(\gamma)}{\Phi(\gamma)}\right)^{2} \text { and } G^{*}(P):=G\left(\omega^{*}(P), P\right)=\left(\frac{\beta_{0} p_{K} f_{G}-P Q}{\Phi(\gamma)}\right)^{2}
$$

(for the latter equalities, cf. Lemma 1a in Appendix B), market clearing in the market for permits requires $e\left(K^{*}(P), G^{*}(P)\right)=a$, which implies $P=P^{*}:=\frac{2 \beta_{0}\left(f_{K} \delta_{K} \gamma+f_{G} \delta_{G} p_{K}\right)-a \Phi(\gamma)}{2 \Psi(\gamma)}$. Note that $P^{*}>0$ if and only if

$$
a<2 \delta_{K} \frac{\beta_{0} \gamma f_{K}}{\Phi(\gamma)}+2 \delta_{G} \frac{\beta_{0} p_{K} f_{G}}{\Phi(\gamma)}
$$

and that the latter condition characterizes a binding permits level in Appendix B, Lemma 2.1 to 2.3. Defining $\omega^{*}:=\omega^{*}\left(P^{*}\right)$, we have that factor inputs equal

$$
K\left(\omega^{*}, P^{*}\right)=\left(\frac{2 \beta_{0} \delta_{G} D+a R(\gamma)}{2 \Psi(\gamma)}\right)^{2} \quad \text { and } \quad G\left(\omega^{*}, P^{*}\right)=\left(\frac{2 \beta_{0} \delta_{K}(-D)+a Q}{2 \Psi(\gamma)}\right)^{2}
$$

respectively, i.e., factor inputs equal those of Lemma $2.1 \mathrm{~b}$ to $2.3 \mathrm{~b}$, where the electricity producer's choice of factor inputs $(K, G) \in \mathbf{R}_{++}$maximizes $\Pi^{(\text {permits })}(K, G) \stackrel{\text { def }}{=} \omega \cdot F(K, G)-p_{K} \cdot K-\gamma$. $G$ subject to $e(K, G) \leq a$ and the permits level is assumed to be binding. 


\section{B Lemmata and Proofs}

Lemma 1 (Equilibria in the Emission Taxes Regime)

In any equilibrium of the emission taxes regime,

a) factor inputs $K$ and $G$ equal $K^{*} \stackrel{\text { def }}{=}\left(\frac{\beta_{0} \gamma f_{K}-p R(\gamma)}{\Phi(\gamma)}\right)^{2} \quad$ and $\quad G^{*} \stackrel{\text { def }}{=}\left(\frac{\beta_{0} p_{K} f_{G}-p Q}{\Phi(\gamma)}\right)^{2}$, respectively, whenever $(p, \gamma)$ satisfies $\beta_{0} \gamma f_{K}-p R(\gamma)>0$ and $\beta_{0} p_{K} f_{G}-p Q>0$.

b) In the monopolistic scenario, gas price $\gamma$ equals

$$
\gamma^{*} \stackrel{\text { def }}{=} \frac{\Phi}{\beta_{1} p_{K}+2 f_{K}^{2}}+\psi
$$

(and is, in particular, independent of the regulator's tax level choice $p$ ) whenever $p<\frac{\beta_{0} \gamma^{*} f_{K}}{R\left(\gamma^{*}\right)}$ and $\beta_{0} p_{K} f_{G}-p Q>0$.

c) Welfare, whenever $(p, \gamma)$ satisfies $\beta_{0} \gamma f_{K}-p R(\gamma)>0$ and $\beta_{0} p_{K} f_{G}-p Q>0$, can be calculated as

$$
W_{\gamma}(p) \stackrel{\text { def }}{=} \frac{\tilde{W}_{2}(\gamma) \cdot p^{2}+\tilde{W}_{1}(\gamma) \cdot p+\tilde{W}_{0}(\gamma)}{2 \beta_{1} \Phi(\gamma)},
$$

where $\tilde{W}_{2}(\gamma) \stackrel{\text { def }}{=}-2 \beta_{1} \Psi(\gamma), \tilde{W}_{1}(\gamma) \stackrel{\text { def }}{=} 4 \beta_{1} q \Psi(\gamma)$, and $\tilde{W}_{0}(\gamma) \stackrel{\text { def }}{=} 2 \beta_{0}\left[\beta_{0}\left(f_{K}^{2} \gamma+f_{G}^{2} p_{K}\right)-2 \beta_{1} q\left(f_{K} \delta_{K} \gamma+\right.\right.$ $\left.\delta_{G} f_{G} p_{K}\right)$ ]. It follows that the regulator's welfare-maximizing tax level choice is $p^{*} \stackrel{\text { def }}{=} q$, i.e., equals the marginal welfare costs of emissions, independent of the scenario.

Refining our representation of $W_{\gamma}(p)$, we obtain that $W_{\gamma}(p) \cdot 2 \beta_{1} \Phi(\gamma)^{2}$ equals

$$
\begin{aligned}
& -\left(2 \beta_{1}\right)^{2}\left(f_{K} \delta_{K} \gamma+f_{G} \delta_{G} p_{K}\right)^{2} \cdot p^{2}-4 \beta_{0} \beta_{1}\left(f_{K} \delta_{K} \gamma+f_{G} \delta_{G} p_{K}\right) \beta_{1} p_{K} \gamma \cdot p \\
& +\left(2 \beta_{0}\right)^{2}\left(f_{K}^{2} \gamma+f_{G}^{2} p_{K}\right)\left(\beta_{1} p_{K} \gamma+f_{K}^{2} \gamma+f_{G}^{2} p_{K}\right) \\
& -\left[2 \beta_{1}\left(p_{K} R(\gamma)^{2}+\gamma Q^{2}\right) \cdot p^{2}-\left(4 \beta_{0} \beta_{1} p_{K} \gamma\left(f_{K} R(\gamma)+f_{G} Q\right)+4 \beta_{1} q \Phi(\gamma)\left(\delta_{K} R(\gamma)+\delta_{G} Q\right)\right) \cdot p\right. \\
& \left.\quad+2 \beta_{1} p_{K} \gamma \beta_{0}^{2}\left(\gamma f_{K}^{2}+p_{K} f_{G}^{2}\right)+4 \beta_{1} q \beta_{0} \Phi(\gamma)\left(f_{K} \delta_{K} \gamma+f_{G} \delta_{G} p_{K}\right)\right],
\end{aligned}
$$

where the first quadratic function of $p$ reflects the utility from electricity consumption and the second quadratic function of $p$ reflects the sum of factor and emission costs.

d) Welfare in the monopolistic and competitive scenario equals

$$
W^{M}(q) \stackrel{\text { def }}{=} \frac{\tilde{W}_{2}^{M} \cdot q^{2}+\tilde{W}_{1}^{M} \cdot q+\tilde{W}_{0}^{M}}{2 \beta_{1} \Phi} \quad \text { and } \quad W^{C}(q) \stackrel{\text { def }}{=} \frac{\tilde{W}_{2}^{C} \cdot q^{2}+\tilde{W}_{1}^{C} \cdot q+\tilde{W}_{0}^{C}}{\beta_{1} \Phi},
$$

respectively, where $\tilde{W}_{0}^{C}:=\beta_{0}^{2}\left(f_{K}^{2} \psi+f_{G}^{2} p_{K}\right), \tilde{W}_{1}^{C}:=-2 \beta_{1} \beta_{0}\left(f_{K} \delta_{K} \psi+f_{G} \delta_{G} p_{K}\right), \tilde{W}_{2}^{C}:=\beta_{1} \Psi$, $\tilde{W}_{0}^{M} \stackrel{\text { def }}{=} \beta_{0}^{2}\left(f_{K}^{2} \psi+f_{G}^{2} p_{K}+\frac{f_{K}^{2} \Phi}{\beta_{1} p_{K}+2 f_{K}^{2}}\right), \tilde{W}_{1}^{M} \stackrel{\text { def }}{=}-2 \beta_{1} \beta_{0}\left(f_{K} \delta_{K} \psi+f_{G} \delta_{G} p_{K}+\frac{f_{K} \delta_{K} \Phi}{\beta_{1} p_{K}+2 f_{K}^{2}}\right)$, and $\tilde{W}_{2}^{M} \stackrel{\text { def }}{=}$ $\beta_{1}\left(\Psi+\frac{\beta_{1} \delta_{K}^{2}}{\beta_{1} p_{K}+2 f_{K}^{2}} \Phi\right)$. Note that

$$
W_{p=0}^{M}(q):=\frac{\tilde{W}_{1}^{M} \cdot q+\tilde{W}_{0}^{M}}{2 \beta_{1} \Phi} \quad \text { and } \quad W_{p=0}^{C}(q):=\frac{\tilde{W}_{1}^{C} \cdot q+\tilde{W}_{0}^{C}}{\beta_{1} \Phi}
$$

equals the resulting welfare in case of no regulation $(p=0)$, respectively. In the monopolistic scenario, the gas supplier's profits are

$$
\Gamma(q) \stackrel{\text { def }}{=}\left(\gamma^{*}-\psi\right) \cdot G^{*}\left(\gamma^{*}, p^{*}\right)=\frac{\left(\beta_{0} p_{K} f_{G}-q Q\right)^{2}}{4 \Phi \cdot\left(\beta_{1} p_{K}+2 f_{K}^{2}\right)}>0 .
$$

Summarizing, $\left(K^{*}\left(p^{*}\right), G^{*}\left(p^{*}\right), p^{*}\right)$ is the unique equilibrium in the competitive scenario, and $\left(K^{*}\left(\gamma^{*}, p^{*}\right), G^{*}\left(\gamma^{*}, p^{*}\right), \gamma^{*}, p^{*}\right)$ is the unique equilibrium in the monopolistic scenario of the emission taxes regime.

\section{Proof of Lemma 1}

a) The electricity producer's profit maximization results in $(K(\omega), G(\omega)) \in \arg \max _{(K, G) \in \mathbf{R}_{++}^{2}}$ $\Pi^{(\text {taxes })}(K, G)$. In particular, $(K(\omega), G(\omega))$ satisfies $\frac{\partial \Pi^{(\text {taxes })}}{\partial K}=\frac{\partial \Pi^{(\text {taxes })}}{\partial G}=0$, which implies $\sqrt{K(\omega)}=\frac{\omega f_{K}-\delta_{K} p}{p_{K}}$ and $\sqrt{G(\omega)}=\frac{\omega f_{G}-\delta_{G} p}{\gamma}$. Market clearing requires $E(\omega)=\beta_{0}-\beta_{1} \omega=$ $2 f_{K} \sqrt{K(\omega)}+2 f_{G} \sqrt{G(\omega)}=F(K(\omega), G(\omega))$, i.e., $\omega=\omega^{*}:=\frac{\beta_{0} p_{K} \gamma+2 f_{K} \delta_{K} p \gamma+2 f_{G} \delta_{G} p_{K} p}{\Phi(\gamma)}$ (and $\left.E\left(\omega^{*}\right)=\frac{2 \beta_{0}\left(f_{K}^{2} \gamma+f_{G}^{2} p_{K}\right)-2 \beta_{1} p\left(f_{K} \delta_{K} \gamma+f_{G} \delta_{G} p_{K}\right)}{\Phi(\gamma)}\right)$. And, $\sqrt{K\left(\omega^{*}\right)}=\frac{\omega^{*} f_{K}-\delta_{K} p}{p_{K}}=\sqrt{K^{*}}$ and $\sqrt{G\left(\omega^{*}\right)}=\frac{\omega^{*} f_{G}-\delta_{G} p}{\gamma}=\sqrt{G^{*}}$. 
b) The gas supplier's profits are well-defined and given by

$$
(\gamma-\psi) \cdot G^{*}(\gamma)=\frac{(\gamma-\psi)\left(\beta_{0} p_{K} f_{G}-p Q\right)^{2}}{\Phi(\gamma)^{2}}
$$

whenever $\beta_{0} p_{K} f_{G}-p Q>0$ and both $p<\frac{\beta_{0} f_{K}}{\beta_{1} \delta_{K}}$ and $\gamma>\frac{2 f_{G}(-D) p}{\beta_{0} f_{K}-\beta_{1} \delta_{K} p}\left(\Leftrightarrow \beta_{0} \gamma f_{K}-p R(\gamma)>\right.$ $\left.0 \Leftrightarrow p<\frac{\beta_{0} \gamma f_{K}}{R(\gamma)}\right)$. Considering the first derivative with respect to gas price $\gamma$, and, in particular, noting that $\left[\left(\beta_{1} p_{K}+2 f_{K}^{2}\right) \gamma+2 f_{G}^{2} p_{K}\right]-(\gamma-\psi) 2\left(\beta_{1} p_{K}+2 f_{K}^{2}\right)=0$ is equivalent to $\gamma=\gamma^{*}$, the profit curve (considered as a function of $\gamma$ ) obtains its maximum at $\gamma^{*}$, it is increasing on $\gamma<\gamma^{*}$ and decreasing on $\gamma>\gamma^{*}$. If $\beta_{0} p_{K} f_{G}-p Q>0$ and both $p<\frac{\beta_{0} f_{K}}{\beta_{1} \delta_{K}}$ and $\gamma^{*}>\frac{2 f_{G}(-D) p}{\beta_{0} f_{K}-\beta_{1} \delta_{K} p}$, then $\gamma^{*}$ maximizes the gas supplier's profits over all alternatives with a well-defined outcome (i.e., over all $\gamma>\frac{2 f_{G}(-D) p}{\beta_{0} f_{K}-\beta_{1} \delta_{K} p}, \gamma \geq \psi$ ). If $\beta_{0} p_{K} f_{G}-p Q>0$, $p<\frac{\beta_{0} f_{K}}{\beta_{1} \delta_{K}}$, and $\gamma^{*} \leq \frac{2 f_{G}(-D) p}{\beta_{0} f_{K}-\beta_{1} \delta_{K} p}$, then the gas supplier's profits cannot be maximized over all alternatives with a well-defined outcome (i.e., over all $\gamma>\frac{2 f_{G}(-D) p}{\beta_{0} f_{K}-\beta_{1} \delta_{K} p}(>\psi)$ ).

c) The calculation of $W_{\gamma}(p)$ is standard (cf. Appendix C). Noting that $\frac{\partial W_{\gamma}(p)}{\partial p}=0$ is equivalent to the tax level being equal to $\frac{-\tilde{W}_{1}(\gamma)}{2 \tilde{W}_{2}(\gamma)}=\frac{-4 \beta_{1} q \Phi(\gamma) \Psi(\gamma)}{-4 \beta_{1} \Psi(\gamma) \Phi(\gamma)}=q, W_{\gamma}(p)$ obtains its maximum at $q$. In the competitive scenario, since $q<\bar{q}$ and (consequently) $\beta_{0} p_{K} f_{G}-q Q>0$, tax level $q$ maximizes $W_{\psi}(p)$ over all $p$ with a well-defined outcome (i.e., over all $p$ with $\beta_{0} p_{K} f_{G}-p Q>0$ and $\left.p<\frac{\beta_{0} f_{K} \psi}{R}\right)$. In the monopolistic scenario, since gas price $\gamma^{*}$ is independent of the regulator's tax level choice $p$, and since $q<\bar{q} \stackrel{\text { def }}{=} \frac{\beta_{0} f_{K} \psi}{R} \leq \frac{\beta_{0} f_{K} \gamma^{*}}{R\left(\gamma^{*}\right)}$ and $\beta_{0} p_{K} f_{G}-q Q>0$, tax level $q$ maximizes $W_{\gamma^{*}}(p)$ over all $p$ with a well-defined outcome (i.e., over all $p$ with $\beta_{0} p_{K} f_{G}-p Q>0$ and $\left.p<\frac{\beta_{0} f_{K} \gamma^{*}}{R\left(\gamma^{*}\right)}\right)$.

d) All welfare levels follow immediately from the representation of $W_{\gamma}(p)$, noting that $\Phi\left(\gamma^{*}\right)=$ $2 \Phi$ and $\Psi\left(\gamma^{*}\right)=\Psi+\frac{\beta_{1} \delta_{K}^{2}}{\beta_{1} p_{K}+2 f_{K}^{2}} \Phi$.

Lemma 2.1 (Necessary Conditions for Equilibria in the Emission Permits Regime if $Q<0$ ) Assuming that any indifference of the gas supplier is resolved according to a preference for a binding (in comparison to a non-binding) emission permits level and that this is common knowledge, in any equilibrium of the emission permits regime in case of $Q<0$,

a) factor inputs $K$ and $G$ equal $K_{A}^{*} \stackrel{\text { def }}{=}\left(\frac{\beta_{0} \gamma f_{K}}{\Phi(\gamma)}\right)^{2}$ and $G_{A}^{*} \stackrel{\text { def }}{=}\left(\frac{\beta_{0} p_{K} f_{G}}{\Phi(\gamma)}\right)^{2}$,

respectively, if the emission permits level is non-binding, and

b) factor inputs $K$ and $G$ equal $K_{B}^{*} \stackrel{\text { def }}{=}\left(\frac{2 \beta_{0} \delta_{G} D+a R(\gamma)}{2 \Psi(\gamma)}\right)^{2}$ and $G_{B}^{*} \stackrel{\text { def }}{=}\left(\frac{2 \beta_{0} \delta_{K}(-D)+a Q}{2 \Psi(\gamma)}\right)^{2}$,

respectively, if the emission permits level is binding and $(\gamma, a)$ satisfies $2 \beta_{0} \delta_{G} D+a R(\gamma)>0$ and $2 \beta_{0} \delta_{K}(-D)+a Q>0$.

c) In the monopolistic scenario, if

$$
a \in\left(a_{-1}:=\frac{2 \beta_{0} \delta_{G}(-D)}{R\left(\gamma_{B}^{*}\right)}, a_{3}:=\frac{2 \beta_{0} \delta_{K} D}{Q}-\frac{2 \beta_{0} p_{K} f_{G}}{-Q} \cdot \sqrt{\frac{\Psi}{\Phi} \cdot \frac{\beta_{1} \delta_{K}^{2}}{\beta_{1} p_{K}+2 f_{K}^{2}}}\right],
$$

then the permits level is binding and gas price $\gamma$ equals $\gamma_{B}^{*} \stackrel{\text { def }}{=} \frac{\Psi}{\beta_{1} \delta_{K}^{2}}+\psi$.
If $a>a_{3}$, then gas price $\gamma$ equals $\gamma_{A}^{*} \stackrel{\text { def }}{=} \frac{\Phi}{\beta_{1} p_{K}+2 f^{2}}+\psi$,

the gas supplier's profits are

and the permits level is non-binding.

$$
\Gamma_{A} \stackrel{\text { def }}{=} \frac{\left(\gamma_{A}^{*}-\psi\right)\left(\beta_{0} p_{K} f_{G}\right)^{2}}{\Phi\left(\gamma_{A}^{*}\right)^{2}}=\frac{\left(\beta_{0} p_{K} f_{G}\right)^{2}}{4 \Phi \cdot\left(\beta_{1} p_{K}+2 f_{K}^{2}\right)}>0
$$

d) In the competitive scenario, where gas price $\gamma$ equals $\psi$, (it is obvious that) permits level $a$ is non-binding if $a \geq a_{1}:=2 \delta_{K} \frac{\beta_{0} \psi f_{K}}{\Phi}+2 \delta_{G} \frac{\beta_{0} p_{K} f_{G}}{\Phi}$ and binding if $a \in\left(\frac{2 \beta_{0} \delta_{G}(-D)}{R}, a_{1}\right)(\neq \emptyset)$. 
e) If the emission permits level is non-binding, then (it is obvious that) the welfare levels equal those of the taxes regime for tax level $p=0$, i.e., $W_{A}^{C}(q)=W_{p=0}^{C}(q)$ and $W_{A}^{M}(q)=W_{p=0}^{M}(q)$ in the competitive and monopolistic scenario, respectively (cf. Lemma 1). In particular, they are below their respective counterparts in the taxes regime (where $p=p^{*}=q>0$ ).

$f)$ If the emission permits level is binding, then welfare, whenever $2 \beta_{0} \delta_{G} D+a R(\gamma)>0$ and $2 \beta_{0} \delta_{K}(-D)+a Q>0\left(\Leftrightarrow a<a_{6}:=\frac{2 \beta_{0} \delta_{K} D}{Q}\right)$, can be calculated as

$$
W_{\gamma}(a) \stackrel{\text { def }}{=} \frac{\tilde{V}_{2}(\gamma) \cdot a^{2}+\tilde{V}_{1}(\gamma) \cdot a+\tilde{V}_{0}(\gamma)}{4 \beta_{1} \Psi(\gamma)},
$$

where $\tilde{V}_{2}(\gamma) \stackrel{\text { def }}{=}-\beta_{1} \Phi(\gamma), \tilde{V}_{1}(\gamma) \stackrel{\text { def }}{=} 4 \beta_{0} \beta_{1}\left(f_{K} \delta_{K} \gamma+f_{G} \delta_{G} p_{K}\right)-4 \beta_{1} q \Psi(\gamma)$, and $\tilde{V}_{0}(\gamma) \stackrel{\text { def }}{=} 4 \beta_{0}^{2} D^{2}$. It follows that the regulator's welfare-maximizing permits level choice, if going for the binding permits level alternative, is

in the competitive and

$$
a_{C}^{*}(q) \stackrel{\text { def }}{=} \frac{2 \beta_{0}\left(f_{K} \delta_{K} \psi+f_{G} \delta_{G} p_{K}\right)-2 q \Psi}{\Phi}=a_{1}-\frac{2 \Psi}{\Phi} q \in\left(\frac{2 \beta_{0} \delta_{G}(-D)}{R}, a_{1}\right)
$$

$$
\hat{a}_{M}^{*}(q) \stackrel{\text { def }}{=} \min \left\{a_{M}^{*}(q) \stackrel{\text { def }}{=} \frac{2 \beta_{0}\left(f_{K} \delta_{K} \psi+f_{G} \delta_{G} p_{K}\right)+\frac{2 \beta_{0} f_{K}}{\beta_{1} \delta_{K}} \Psi-4 q \Psi}{\Phi+\frac{\beta_{1} p_{K}+2 f_{K}^{2}}{\beta_{1} \delta_{K}^{2}} \Psi}, a_{3}\right\} \in\left(a_{-1}, a_{3}\right]
$$

in the monopolistic scenario.

In particular, the regulator's permits level choice is higher in the monopolistic as compared to the competitive scenario. And,

equals $a_{3}$ at

$$
a_{M}^{*}(q)=a_{4}-\frac{4 \Psi}{\Phi+\frac{\beta_{1} p_{K}+2 f_{K}^{2}}{\beta_{1} \delta_{K}^{2}} \Psi} q
$$

$$
q=q_{3}:=\frac{\beta_{0} p_{K} f_{G}}{2 \Psi \cdot(-Q)} \cdot\left[\sqrt{\frac{\Psi}{\Phi} \cdot \frac{\beta_{1} \delta_{K}^{2}}{\beta_{1} p_{K}+2 f_{K}^{2}}} \cdot\left(\Phi+\frac{\beta_{1} p_{K}+2 f_{K}^{2}}{\beta_{1} \delta_{K}^{2}} \Psi\right)-2 \Psi\right]>0 .
$$

If the regulator goes for the binding permits level alternative, then welfare in the competitive scenario $\left(\gamma=\psi, a=a_{C}^{*}\right)$ equals

$$
W_{B}^{C}(q) \stackrel{\text { def }}{=} \frac{1}{\beta_{1} \Psi \Phi}\left[\beta_{1}\left(\beta_{0}\left(f_{K} \delta_{K} \psi+f_{G} \delta_{G} p_{K}\right)-q \Psi\right)^{2}+\beta_{0}^{2} D^{2} \Phi\right] .
$$

In the monopolistic scenario $\left(\gamma=\gamma_{B}^{*}, a=\hat{a}_{M}^{*}\right.$ ), if $q \geq q_{3}$ (and thus $\hat{a}_{M}^{*}=a_{M}^{*}$ ), welfare equals

$$
W_{B}^{M}(q) \stackrel{\text { def }}{=} \frac{\beta_{1}\left[\beta_{0}\left(f_{K} \delta_{K} \psi+f_{G} \delta_{G} p_{K}\right)+\frac{\beta_{0} f_{K}}{\beta_{1} \delta_{K}} \Psi-2 q \Psi\right]^{2}+\beta_{0}^{2} D^{2}\left(\Phi+\frac{\beta_{1} p_{K}+2 f_{K}^{2}}{\beta_{1} \delta_{K}^{2}} \Psi\right)}{2 \beta_{1} \Psi \cdot\left(\Phi+\frac{\beta_{1} p_{K}+2 f_{K}^{2}}{\beta_{1} \delta_{K}^{2}} \Psi\right)},
$$

and the gas supplier's profits are

$$
\Gamma_{B}(q) \stackrel{\text { def }}{=}\left(\gamma_{B}^{*}-\psi\right) \cdot \frac{\left[2 \beta_{0} \delta_{K}(-D)+Q a_{M}^{*}\right]^{2}}{4 \Psi\left(\gamma_{B}^{*}\right)^{2}}=\frac{\beta_{1} \delta_{K}^{2} \Psi \cdot\left(\beta_{0} f_{G} p_{K}-q Q\right)^{2}}{\left[\beta_{1} \delta_{K}^{2} \Phi+\left(\beta_{1} p_{K}+2 f_{K}^{2}\right) \Psi\right]^{2}}>0 .
$$

If $q<q_{3}$ (and thus $\hat{a}_{M}^{*}=a_{3}<a_{M}^{*}$ ), welfare is no higher than $W_{B}^{M}(q)$, and the gas supplier's profits are

$$
\left(\gamma_{B}^{*}-\psi\right) \cdot \frac{\left[2 \beta_{0} \delta_{K}(-D)+Q a_{3}\right]^{2}}{4 \Psi\left(\gamma_{B}^{*}\right)^{2}}=\Gamma_{A}>0
$$

g) In the competitive scenario, $W_{B}^{C}(q)>W_{A}^{C}(q)$. Therefore, the regulator's welfare-maximizing permits level choice is $a_{C}^{*}$, and the emission permits level is binding.

h) In the monopolistic scenario, $W_{A}^{M}(q) \stackrel{<}{>} W_{B}^{M}(q) \Leftrightarrow q \stackrel{\gtrless}{\gtrless}(Q) \stackrel{\text { def }}{=} \frac{\beta_{0} p_{K} f_{G}(-Q)}{2 \beta_{1} \delta_{K}^{2} \Phi}\left(>q_{3}\right)$. Therefore, if $q>\tilde{q}(Q)$, then the regulator's welfare-maximizing permits level choice is $\hat{a}_{M}^{*}=$ $a_{M}^{*}$, the emission permits level is binding, and the welfare level equals $W_{B}^{M}(q)$. If $q<\tilde{q}(Q)$, any permits level above $a_{3}$ maximizes welfare and, in particular, is non-binding, and the welfare level equals $W_{A}^{M}(q)$. If $q=\tilde{q}(Q)$, then welfare is maximized by choosing either $\hat{a}_{M}^{*}=a_{M}^{*}$ (resulting in a binding permits level) or any level above $a_{3}$ (resulting in a nonbinding permits level), and the welfare level equals $W_{B}^{M}(q)=W_{A}^{M}(q)$. 


\section{Proof of Lemma 2.1}

a) The electricity producer's profit maximization results in $(K(\omega), G(\omega)) \in \arg \max _{(K, G) \in \mathbf{R}_{++}^{2}}$ $\Pi_{p=0}^{(\text {taxes })}(K, G), \Pi_{p=0}^{(\text {taxes })}(K, G):=\omega F(K, G)-p_{K} K-\gamma G$. In particular, $(K(\omega), G(\omega))$ satisfies $\frac{\partial \Pi_{p=0}^{(\text {taxes })}}{\partial K}=0$ and $\frac{\partial \Pi_{p=0}^{(\text {taxes })}}{\partial G}=0$, which implies $\sqrt{K(\omega)}=\frac{\omega f_{K}}{p_{K}}$ and $\sqrt{G(\omega)}=\frac{\omega f_{G}}{\gamma}$. Market clearing requires $E(\omega)=\beta_{0}-\beta_{1} \omega=2 f_{K} \sqrt{K(\omega)}+2 f_{G} \sqrt{G(\omega)}=F(K(\omega), G(\omega))$, i.e., $\omega=\omega_{A}^{*}:=\frac{\beta_{0} p_{K} \gamma}{\Phi(\gamma)}$ (and $E\left(\omega_{A}^{*}\right)=\frac{2 \beta_{0}\left(f_{K}^{2} \gamma+f_{G}^{2} p_{K}\right)}{\Phi(\gamma)}$ ). And, $\sqrt{K\left(\omega_{A}^{*}\right)}=\frac{\omega_{A}^{*} f_{K}}{p_{K}}=\sqrt{K_{A}^{*}}$ and $\sqrt{G\left(\omega_{A}^{*}\right)}=\frac{\omega_{A}^{*} f_{G}}{\gamma}=\sqrt{G_{A}^{*}}$.

b) The electricity producer's profit maximization results in $(K(\omega), G(\omega)) \in \arg \max _{(K, G) \in \mathbf{R}_{++}^{2}}$ $\Pi^{\text {(permits) }}(K, G)$ subject to $e(K, G) \leq a$. In particular, there exists a multiplier $\lambda \in \mathbf{R}_{++}$ such that $\frac{\partial \Pi^{(\text {permits })}}{\partial K}=\lambda \frac{\partial e}{\partial K}$ and $\frac{\partial \Pi^{(p e r m i t s)}}{\partial G}=\lambda \frac{\partial e}{\partial G}$, which implies $\sqrt{K(\omega)}=\frac{\omega f_{K}-\delta_{K} \lambda}{p_{K}}$ and $\sqrt{G(\omega)}=\frac{\omega f_{G}-\delta_{G} \lambda}{\gamma}$. Since $e(K(\omega), G(\omega))=a$, we have that $2 \delta_{K} \gamma\left(\omega f_{K}-\delta_{K} \lambda\right)+2 \delta_{G} p_{K}\left(\omega f_{G}-\right.$ $\left.\delta_{G} \lambda\right)=a p_{K} \gamma$, which is equivalent to $\lambda=\frac{2 \delta_{K} \gamma \omega f_{K}+2 \delta_{G} p_{K} \omega f_{G}-a p_{K} \gamma}{2 \delta_{K}^{2} \gamma+2 \delta_{G}^{2} p_{K}}$. Thus, $\sqrt{K(\omega)}=$ $\frac{\omega f_{K}-\delta_{K} \lambda}{p_{K}}=\frac{\omega f_{K} 2 \delta_{G}^{2}-2 \delta_{G} \delta_{K} \omega f_{G}+a \delta_{K} \gamma}{2 \delta_{K}^{2} \gamma+2 \delta_{G}^{2} p_{K}}, \sqrt{G(\omega)}=\frac{\omega f_{G}-\delta_{G} \lambda}{\gamma}=\frac{\omega f_{G} 2 \delta_{K}^{2}-2 \delta_{G} \delta_{K} \omega f_{K}+a \delta_{G} p_{K}}{2 \delta_{K}^{2} \gamma+2 \delta_{G}^{2} p_{K}}$, and market clearing requires $E(\omega)=\beta_{0}-\beta_{1} \omega=2 f_{K} \sqrt{K(\omega)}+2 f_{G} \sqrt{G(\omega)}=F(K(\omega), G(\omega))$, i.e., $\omega=\omega_{B}^{*}:=\frac{\beta_{0}\left(\delta_{K}^{2} \gamma+\delta_{G}^{2} p_{K}\right)-a\left(f_{K} \delta_{K} \gamma+f_{G} \delta_{G} p_{K}\right)}{\Psi(\gamma)}$ (and $E\left(\omega_{B}^{*}\right)=\frac{2 \beta_{0} D^{2}+\beta_{1} a\left(f_{K} \delta_{K} \gamma+f_{G} \delta_{G} p_{K}\right)}{\Psi(\gamma)}$ ). It follows that $K\left(\omega_{B}^{*}\right)=K_{B}^{*}$ and $G\left(\omega_{B}^{*}\right)=G_{B}^{*}$.

c) First, note that the gas supplier's profits in case of a binding permits level are given by

$$
(\gamma-\psi) \cdot G_{B}^{*}(\gamma)=\frac{(\gamma-\psi)\left[2 \beta_{0} \delta_{K}(-D)+a Q\right]^{2}}{4 \Psi(\gamma)^{2}} .
$$

Considering the first derivative with respect to gas price $\gamma$, and, in particular, noting that $\left[\beta_{1}\left(\delta_{K}^{2} \gamma+\delta_{G}^{2} p_{K}\right)+2 D^{2}\right]-(\gamma-\psi) 2 \beta_{1} \delta_{K}^{2}=0$ is equivalent to $\gamma=\gamma_{B}^{*}$, the profit curve (considered as a function of $\gamma$ ) obtains its maximum at $\gamma_{B}^{*}$, it is increasing on $\gamma<\gamma_{B}^{*}$ and decreasing on $\gamma>\gamma_{B}^{*}$. For $\gamma=\gamma_{B}^{*}$, the profit curve (considered as a function of $a$ ) equals $C_{B}(\cdot)$, $C_{B}(a):=\frac{\Psi}{\beta_{1} \delta_{K}^{2}} \cdot \frac{\left[2 \beta_{0} \delta_{K}(-D)+a Q\right]^{2}}{4(2 \Psi)^{2}}$, noting that $\Psi\left(\gamma_{B}^{*}\right)=2 \Psi$ and $\Phi\left(\gamma_{B}^{*}\right)=\Phi+\frac{\beta_{1} p_{K}+2 f_{K}^{2}}{\beta_{1} \delta_{K}^{2}} \Psi$. Curve $C_{B}(\cdot)$ is a quadratic function of $a$, it obtains its minimum value 0 at $a_{6} \stackrel{\text { def }}{=} \frac{2 \beta_{0} \delta_{K} D}{Q}$, it is decreasing on $a<a_{6}$ and increasing on $a>a_{6}$.

The gas supplier's profits in case of a non-binding permits level are given by

$$
(\gamma-\psi) \cdot G_{A}^{*}(\gamma)=\frac{(\gamma-\psi) \cdot\left(\beta_{0} p_{K} f_{G}\right)^{2}}{\Phi(\gamma)^{2}}
$$

This curve (considered as a function of $\gamma$ ) obtains its maximum at $\gamma_{A}^{*}$, it is increasing on $\gamma<\gamma_{A}^{*}$ and decreasing on $\gamma>\gamma_{A}^{*}$ (cf. the proof of Lemma 1b). For $\gamma=\gamma_{A}^{*}$, this curve (considered as a function of $a$ ) is constant in $a$ and equals $\bar{C}_{A}:=\frac{\Phi}{\left(\beta_{1} p_{K}+2 f_{K}^{2}\right)} \cdot \frac{\left(\beta_{0} p_{K} f_{G}\right)^{2}}{(2 \Phi)^{2}}$.

If going for the binding permits level alternative, profit is maximized by choosing $\gamma(a) \in$ $\arg \max _{\gamma \in[\psi, \infty)}(\gamma-\psi) \cdot G_{B}^{*}(\gamma)$ subject to $2 \delta_{K} \sqrt{K_{A}^{*}}+2 \delta_{G} \sqrt{G_{A}^{*}}>a$, i.e., subject to the permits level being binding (cf. part a) of Lemma 2.1). First, note that going for the binding permits level alternative requires $a<a_{5}:=\frac{2 \beta_{0} f_{K} \delta_{K}}{\beta_{1} p_{K}+2 f_{K}^{2}}$, since otherwise the inequality constraint cannot be satisfied for $\gamma \geq 0$ : If $a>a_{5}$, then the inequality constraint is equivalent to $\gamma<\hat{\gamma}(a)$,

$$
\hat{\gamma}(a) \stackrel{\text { def }}{=} \frac{a \cdot 2 f_{G}^{2} p_{K}-2 \beta_{0} f_{G} \delta_{G} p_{K}}{2 \beta_{0} \delta_{K} f_{K}-a \cdot\left(\beta_{1} p_{K}+2 f_{K}^{2}\right)},
$$

the fraction on the right-hand side being lower than zero (since the numerator is positive and the denominator negative), and if $a=a_{5}$, then the inequality constraint is equivalent to $-Q<0$, contradicting $Q<0$. Note that, in particular, since $a_{5}<a_{6}$ (cf. Appendix C), $a<a_{5}$ implies that $a<a_{6}$, which is equivalent to $2 \beta_{0} \delta_{K}(-D)+a Q>0$. If $a<a_{5}$, then the inequality constraint $2 \delta_{K} \frac{\beta_{0} \gamma f_{K}}{\Phi(\gamma)}+2 \delta_{G} \frac{\beta_{0} p_{K} f_{G}}{\Phi(\gamma)}>a$ is equivalent to $\gamma>\hat{\gamma}(a)$. If $a<a_{4}:=$ $2 \delta_{K} \frac{\beta_{0} \gamma_{B}^{*} f_{K}}{\Phi\left(\gamma_{B}^{*}\right)}+2 \delta_{G} \frac{\beta_{0} p_{K} f_{G}}{\Phi\left(\gamma_{B}^{*}\right)}=\left[2 \beta_{0}\left(f_{K} \delta_{K} \psi+f_{G} \delta_{G} p_{K}\right)+2 \frac{\beta_{0} f_{K}}{\beta_{1} \delta_{K}} \Psi\right] \cdot\left(\Phi+\frac{\beta_{1} p_{K}+2 f_{K}^{2}}{\beta_{1} \delta_{K}^{2}} \Psi\right)^{-1}\left(<a_{5}, \mathrm{cf}\right.$. 
Appendix C), and if $a>a_{-1} \stackrel{\text { def }}{=} \frac{2 \beta_{0} \delta_{G}(-D)}{R\left(\gamma_{B}^{*}\right)}=\frac{2 \beta_{0} \delta_{G}(-D)}{R+\frac{\Psi}{\delta_{K}}}\left(<a_{0}\right.$, cf. Appendix C), then the gas supplier's profit (if going for the binding alternative) is maximized over all alternatives with a well-defined outcome (i.e., over all $\left.\gamma>\frac{2(-D)\left(\beta_{0} \delta_{G}-f_{G} a\right)}{\beta_{1} \delta_{K} a}, \gamma \geq \psi\right)$ by choosing $\gamma(a)=\gamma_{B}^{*}$, and his corresponding profit is $C_{B}(a)$; if $a \in\left[a_{4}, a_{5}\right)$, then obviously the gas supplier's achievable profit cannot be higher than $C_{B}(a)$. If $a \leq a_{-1}$, then the gas supplier's profit (if going for the binding alternative) cannot be maximized over all alternatives with a welldefined outcome (i.e., over all $\gamma>\frac{2(-D)\left(\beta_{0} \delta_{G}-f_{G} a\right)}{\beta_{1} \delta_{K} a}(>\psi)$ ).

If going for the non-binding permits level alternative, profit is maximized by choosing $\gamma(a) \in$ $\arg \max _{\gamma \in[\psi, \infty)}(\gamma-\psi) \cdot G_{A}^{*}(\gamma)$ subject to $2 \delta_{K} \sqrt{K_{A}^{*}}+2 \delta_{G} \sqrt{G_{A}^{*}} \leq a$, i.e., subject to the permits level being non-binding. First, note that the inequality constraint is satisfied for all $\gamma \geq 0$ if $a \geq a_{5}$. If $a<a_{5}$, then the constraint cannot be satisfied for $\gamma>0$ if $a \leq a_{0} \stackrel{\text { def }}{=} \frac{\beta_{0} \delta_{G}}{f_{G}}\left(<a_{5}\right.$, cf. Appendix C), and the constraint is equivalent to $\gamma \leq \hat{\gamma}(a)(>0)$ if $a>a_{0}$. In the latter case, i.e., $a \in\left(a_{0}, a_{5}\right)$, the interval of available gas prices is bounded from above by an increasing function of permits level $a$ (cf. Appendix C), that allows the gas supplier to choose (gas price $\psi$ exactly on $a \geq a_{1} \stackrel{\text { def }}{=} 2 \delta_{K} \frac{\beta_{0} \psi f_{K}}{\Phi}+2 \delta_{G} \frac{\beta_{0} p_{K} f_{G}}{\Phi}$, and) gas price $\gamma_{A}^{*}$ exactly on $a \geq a_{2}:=2 \delta_{K} \frac{\beta_{0} \gamma_{A}^{*} f_{K}}{\Phi\left(\gamma_{A}^{*}\right)}+2 \delta_{G} \frac{\beta_{0} p_{K} f_{G}}{\Phi\left(\gamma_{A}^{*}\right)}=\frac{\beta_{0}}{\Phi}\left(f_{G} \delta_{G} p_{K}+f_{K} \delta_{K} \psi+\frac{f_{K} \delta_{K}}{\beta_{1} p_{K}+2 f_{K}^{2}} \Phi\right)$. Since $0<a_{-1}<a_{0}<a_{1}<a_{2}<a_{4}<a_{5}<a_{6}$ (cf. Appendix C), it follows that the gas supplier's profit (if going for the non-binding alternative) is maximized by choosing $\gamma(a)=\hat{\gamma}(a)$ on $a \in\left(a_{0}, a_{2}\right]$ and $\gamma(a)=\gamma_{A}^{*}$ on $a \geq a_{2}$, and his corresponding profit is lower than $\bar{C}_{A}$ on $a \in\left(a_{0}, a_{2}\right)$ and equals $\bar{C}_{A}$ on $a \geq a_{2}$.

Curve $C_{B}(\cdot)$ equals $\bar{C}_{A}$ at both $a_{3}$ and $a_{7}:=a_{6}+\frac{2 \beta_{0} p_{K} f_{G}}{-Q} \cdot \sqrt{\frac{\Psi}{\Phi} \cdot \frac{\beta_{1} \delta_{K}^{2}}{\beta_{1} p_{K}+2 f_{K}^{2}}}>a_{6}$ (cf. Appendix C). Since $a_{3} \in\left(a_{2}, a_{4}\right)$ (cf. Appendix C), $\bar{C}_{A}$ lies above $C_{B}(\cdot)$ between $a_{3}$ and $a_{7}$, and $\bar{C}_{A}$ lies below $C_{B}(\cdot)$ outside $\left[a_{3}, a_{7}\right]$. In particular, given our assumption concerning the gas supplier's preference for a binding permits level (if indifferent with respect to profits), the gas supplier goes for the binding alternative if $a \in\left(a_{-1}, a_{3}\right]$ and for the non-binding alternative if $a>a_{3}$.

d) It is easy to verify that $\frac{2 \beta_{0} \delta_{G}(-D)}{R}<a_{1}$ (cf. Appendix C).

f) The calculation of $W_{\gamma}(a)$ is standard (cf. Appendix C). Considering the first derivative with respect to permits level $a$, and, in particular, noting that $\frac{\partial W_{\gamma}(a)}{\partial a}=0$ is equivalent to the permits level being equal to $a^{*}(\gamma):=\frac{-\tilde{V}_{1}(\gamma)}{2 \tilde{V}_{2}(\gamma)}=\frac{2 \beta_{0}\left(f_{K} \delta_{K} \gamma+f_{G} \delta_{G} p_{K}\right)-2 q \Psi(\gamma)}{\Phi(\gamma)}$,

$W_{\gamma}(a)$ obtains its maximum at $a^{*}(\gamma)$, it is increasing on $a<a^{*}(\gamma)$ and decreasing on $a>a^{*}(\gamma)$. It is easy to verify that $a^{*}\left(\gamma_{B}^{*}\right)>a^{*}(\psi)>\frac{2 \beta_{0} \delta_{G}(-D)}{R}>0$ (cf. Appendix C), that $a^{*}\left(\gamma_{B}^{*}\right) \stackrel{(>)}{=} a_{3} \Leftrightarrow q \stackrel{(<)}{=} q_{3}$, and that $q_{3}>0$ (cf. Appendix C). Since $a^{*}\left(\gamma_{B}^{*}\right)$ and $a_{3}$ are both greater than $\frac{2 \beta_{0} \delta_{G}(-D)}{R\left(\gamma_{B}^{*}\right)}$ (cf. Appendix C), $a^{*}(\psi)>\frac{2 \beta_{0} \delta_{G}(-D)}{R}$, and $a^{*}(\psi)<a_{1}$, and given our previous results for the two scenarios, we have that the welfare-maximizing permits level equals $\min \left\{a^{*}\left(\gamma_{B}^{*}\right)=a_{M}^{*}, a_{3}\right\}$ in the monopolistic and $a^{*}(\psi)=a_{C}^{*}$ in the competitive scenario. To see that $a_{C}^{*}(q)<\hat{a}_{M}^{*}(q)$, note that $a^{*}\left(\gamma_{B}^{*}\right) \leq a_{3}$ implies $a^{*}(\psi)<a^{*}\left(\gamma_{B}^{*}\right)=$ $\min \left\{a^{*}\left(\gamma_{B}^{*}\right), a_{3}\right\}$, and that $a^{*}\left(\gamma_{B}^{*}\right) \geq a_{3}$ implies $a^{*}(\psi)<a_{1}<a_{3}=\min \left\{a^{*}\left(\gamma_{B}^{*}\right), a_{3}\right\}$.

All welfare implications follow immediately from the representation of $W_{\gamma}(a)$. The calculations for the gas supplier's profits are standard (cf. Appendix C).

g) The relation between $W_{B}^{C}(q)$ and $W_{A}^{C}(q)$ is easy to verify (cf. Appendix C).

h) The relation between $q_{3}$ and $\tilde{q}(Q)$ and the relationship between $W_{B}^{M}(q)$ and $W_{A}^{M}(q)$ on the one hand and $q$ and $\tilde{q}(Q)$ on the other hand are both easy to verify (cf. Appendix C). 
Lemma 2.2 (Necessary Conditions for Equilibria in the Emission Permits Regime if $Q>0$ ) In any equilibrium of the emission permits regime in case of $Q>0$,

a) factor inputs $K$ and $G$ equal $K_{A}^{*}$ and $G_{A}^{*}$ as specified in Lemma 2.1a, respectively, if the emission permits level is non-binding, and

b) factor inputs $K$ and $G$ equal $K_{B}^{*}$ and $G_{B}^{*}$ as specified in Lemma 2.1b, respectively, if the emission permits level is binding and $(\gamma, a)$ satisfies $2 \beta_{0} \delta_{G} D+a R(\gamma)>0$.

c) In the monopolistic scenario, if $a \in\left(a_{-1} \stackrel{\text { def }}{=} \frac{2 \beta_{0} \delta_{G}(-D)}{R\left(\gamma_{B}^{*}\right)}, a_{4} \stackrel{\text { def }}{=} 2 \delta_{K} \frac{\beta_{0} \gamma_{B}^{*} f_{K}}{\Phi\left(\gamma_{B}^{*}\right)}+2 \delta_{G} \frac{\beta_{0} p_{K} f_{G}}{\Phi\left(\gamma_{B}^{*}\right)}\right)$, then the permits level is binding and gas price $\gamma$ equals $\gamma_{B}^{*} \stackrel{\text { def }}{=} \frac{\Psi}{\beta_{1} \delta_{K}^{2}}+\psi$. If $a \geq a_{2}$, then the permits level is non-binding, gas price $\gamma$ equals $\gamma_{A}^{*} \stackrel{\text { def }}{=} \frac{\Phi}{\beta_{1} p_{K}+2 f_{K}^{2}}+\psi$, and the gas supplier's profits are $\Gamma_{A}$ as specified in Lemma 2.1c. If $a \in\left[a_{4}, a_{2} \stackrel{\text { def }}{=} \frac{\beta_{0}}{\Phi} \cdot\left(f_{G} \delta_{G} p_{K}+f_{K} \delta_{K} \psi+\frac{f_{K} \delta_{K}}{\beta_{1} p_{K}+2 f_{K}^{2}} \Phi\right)\right]$, then the permits level is non-binding, gas price $\gamma$ equals

and the gas supplier's profits are

$$
\hat{\gamma}(a) \stackrel{\text { def }}{=} \frac{a \cdot 2 f_{G}^{2} p_{K}-2 \beta_{0} f_{G} \delta_{G} p_{K}}{2 \beta_{0} \delta_{K} f_{K}-a \cdot\left(\beta_{1} p_{K}+2 f_{K}^{2}\right)},
$$

$$
\hat{\Gamma}_{A}(a):=\frac{\left[\Phi \cdot a-2 \beta_{0}\left(f_{K} \delta_{K} \psi+f_{G} \delta_{G} p_{K}\right)\right] \cdot\left[2 \beta_{0} f_{K} \delta_{K}-\left(\beta_{1} p_{K}+2 f_{K}^{2}\right) \cdot a\right]}{4 Q^{2}}>0
$$

(note that $\hat{\gamma}\left(a_{2}\right)=\gamma_{A}^{*}$ and $\left.\hat{\gamma}\left(a_{4}\right)=\gamma_{B}^{*}\right)$.

d) In the competitive scenario, where gas price $\gamma$ equals $\psi$, (it is obvious that) permits level a is non-binding if $a \geq a_{1} \stackrel{\text { def }}{=} 2 \delta_{K} \frac{\beta_{0} \psi f_{K}}{\Phi}+2 \delta_{G} \frac{\beta_{0} p_{K} f_{G}}{\Phi}$ and binding if $a \in\left(\frac{2 \beta_{0} \delta_{G}(-D)}{R}, a_{1}\right)$.

e) In the competitive scenario, if the emission permits level is non-binding, then (it is obvious that) the welfare level equals that of the taxes regime for tax level $p=0$, i.e., $W_{A}^{C}(q)=$ $W_{p=0}^{C}(q)$ (cf. Lemma 1). In particular, the welfare level is below its counterpart in the emission taxes regime (where $p=p^{*}=q$ ).

In the monopolistic scenario, if the emission permits level is non-binding and $a \geq a_{2}$, then the welfare level equals that of the taxes regime for tax level $p=0$, i.e., $W_{A}^{M}(q)=W_{p=0}^{M}(q)$ (cf. Lemma 1). In particular, the welfare level is below its counterpart in the taxes regime (where $p=p^{*}=q$ ). If the emission permits level is non-binding and $a \in\left[a_{4}, a_{2}\right]$, then welfare can be calculated as

(cf. Lemma 1c).

$$
\hat{W}^{M}(a):=\frac{\tilde{W}_{0}(\hat{\gamma}(a))}{2 \beta_{1} \Phi(\hat{\gamma}(a))}=\frac{2 \beta_{0}^{2} f_{K} D+\beta_{1}\left(\beta_{0} f_{G} p_{K}-2 Q q\right) \cdot a}{2 \beta_{1} Q}
$$

It follows that the regulator's welfare maximizing permits level choice in the monopolistic scenario, if going for the non-binding permits level alternative, is $a_{4}$ if $q>\frac{\beta_{0} f_{G} p_{K}}{2 Q}$, resulting in welfare level $\hat{W}_{A}^{M}(q):=\hat{W}^{M}\left(a_{4}\right)\left(>\hat{W}^{M}\left(a_{2}\right)=W_{A}^{M}(q)\right)$. If $q<\frac{\beta_{0} f_{G} p_{K}}{2 Q}$ or $q=\frac{\beta_{0} f_{G} p_{K}}{2 Q}$, then any $a \geq a_{2}$ or any $a \geq a_{4}$, respectively, maximizes welfare, resulting in welfare $W_{A}^{M}(q)$.

$f)$ If the emission permits level is binding, then welfare, whenever $2 \beta_{0} \delta_{G} D+a R(\gamma)>0$, equals $W_{\gamma}(a)$ as specified in Lemma 2.1f. It follows that the regulator's welfare-maximizing permits level choice, if going for the binding permits level alternative, is

$$
a_{C}^{*}(q) \stackrel{\text { def }}{=} a_{1}-\frac{2 \Psi}{\Phi} q \in\left(\frac{2 \beta_{0} \delta_{G}(-D)}{R}, a_{1}\right) \quad \text { and } a_{M}^{*}(q) \stackrel{\text { def }}{=} a_{4}-\frac{4 \Psi}{\Phi+\frac{\beta_{1} p_{K}+2 f_{K}^{2}}{\beta_{1} \delta_{K}^{2}} \Psi} q \in\left(a_{-1}, a_{4}\right)
$$

in the competitive and in the monopolistic scenario, respectively.

In particular, the regulator's permits level choice is lower in the monopolistic as compared to the competitive scenario.

If the regulator goes for the binding permits level alternative, then welfare in the competitive scenario $\left(\gamma=\psi, a=a_{C}^{*}\right)$ equals $W_{B}^{C}(q)$ as specified in Lemma 2.1f. In the monopolistic scenario $\left(\gamma=\gamma_{B}^{*}, a=a_{M}^{*}\right)$, welfare equals $W_{B}^{M}(q)$ as specified in Lemma 2.1f, and the gas supplier's profits are $\Gamma_{B}(q)$ as specified in Lemma 2.1f.

$g$ ) In the competitive scenario, $W_{B}^{C}(q)>W_{A}^{C}(q)$. Therefore, the regulator's welfare-maximizing permits level choice is $a_{C}^{*}$, and the emission permits level is binding. 
h) In the monopolistic scenario, $W_{A}^{M}(q)>W_{B}^{M}(q) \Leftrightarrow q \gtrless \tilde{q}(Q) \stackrel{\text { def }}{=} \frac{\beta_{0} p_{K} f_{G} Q}{2\left(\beta_{1} p_{K}+2 f_{K}^{2}\right) \Psi}\left(<\frac{\beta_{0} f_{G} p_{K}}{2 Q}\right)$, and $\hat{W}_{A}^{M}(q)<W_{B}^{M}(q)$. Therefore, if $q>\tilde{q}(Q)$, then the regulator's welfare-maximizing permits level choice is $a_{M}^{*}$, the emission permits level is binding, and the welfare level equals $W_{B}^{M}(q)$. If $q<\tilde{q}(Q)$, any permits level $a \geq a_{2}$ maximizes welfare and, in particular, is non-binding, and the welfare level equals $W_{A}^{M}(q)$. If $q=\tilde{q}(Q)$, then welfare is maximized by choosing either $a_{M}^{*}$ (resulting in a binding permits level) or any level $a \geq a_{2}$ (resulting in a non-binding permits level), and the welfare level equals $W_{B}^{M}(q)=W_{A}^{M}(q)$.

\section{Proof of Lemma 2.2}

c) First, note that the gas supplier's profits in case of a binding permits level are given by $(\gamma-\psi) \cdot G_{B}^{*}(\gamma)$ as specified in the proof of Lemma 2.1c. This curve (considered as a function of $\gamma$ ) obtains its maximum at $\gamma_{B}^{*}$ and, for $\gamma=\gamma_{B}^{*}$, this curve (considered as a function of a) equals $C_{B}(\cdot), C_{B}(a) \stackrel{\text { def }}{=} \frac{\Psi}{\beta_{1} \delta_{K}^{2}} \cdot \frac{\left[2 \beta_{0} \delta_{K}(-D)+a Q\right]^{2}}{4(2 \Psi)^{2}}$. Curve $C_{B}(\cdot)$ is a quadratic function of $a$, it is increasing and positive on $a \geq 0$. The gas supplier's profits in case of a non-binding permits level are given by $(\gamma-\psi) \cdot G_{A}^{*}(\gamma)$ as specified in the proof of Lemma 2.1c. This curve (considered as a function of $\gamma$ ) obtains its maximum at $\gamma_{A}^{*}$ and, for $\gamma=\gamma_{A}^{*}$, this curve (considered as a function of $a$ ) is constant in $a$ and equals $\bar{C}_{A} \stackrel{\text { def }}{=} \frac{\Phi}{\left(\beta_{1} p_{K}+2 f_{K}^{2}\right)} \cdot \frac{\left(\beta_{0} p_{K} f_{G}\right)^{2}}{(2 \Phi)^{2}}$. (Cf. the proof of Lemma 2.1c.)

Note that, since $Q>0$, now $0<a_{5}<a_{4}<a_{2}<a_{1}<a_{0}$ and $a_{-1}<a_{4}$ (cf. Appendix C). If going for the non-binding permits level alternative, profit is maximized by choosing $\gamma(a) \in$ $\arg \max _{\gamma \in[\psi, \infty)}(\gamma-\psi) \cdot G_{A}^{*}(\gamma)$ subject to $2 \delta_{K} \sqrt{K_{A}^{*}}+2 \delta_{G} \sqrt{G_{A}^{*}} \leq a$, i.e., subject to the permits level being non-binding (cf. part a) of Lemma 2.2). First, note that going for the non-binding permits level alternative requires $a>a_{5} \stackrel{\text { def }}{=} \frac{2 \beta_{0} f_{K} \delta_{K}}{\beta_{1} p_{K}+2 f_{K}^{2}}$, since otherwise the inequality constraint cannot be satisfied for $\gamma \geq 0$ : If $a<a_{5}$, then the inequality constraint is equivalent to $\gamma \leq \hat{\gamma}(a) \stackrel{\text { def }}{=} \frac{a \cdot 2 f_{G}^{2} p_{K}-2 \beta_{0} f_{G} \delta_{G} p_{K}}{2 \beta_{0} \delta_{K} f_{K}-a \cdot\left(\beta_{1} p_{K}+2 f_{K}^{2}\right)}$, the fraction on the right-hand side being lower than zero (since the numerator is negative and the denominator positive), and if $a=a_{5}$, then the inequality constraint is equivalent to $Q \leq 0$, contradicting $Q>0$. If $a>a_{5}$, then the inequality constraint $2 \delta_{K} \frac{\beta_{0} \gamma f_{K}}{\Phi(\gamma)}+2 \delta_{G} \frac{\beta_{0} p_{K} f_{G}}{\Phi(\gamma)} \leq a$ is equivalent to $\gamma \geq \hat{\gamma}(a)$. If $a \geq a_{2} \stackrel{\text { def }}{=} 2 \delta_{K} \frac{\beta_{0} \gamma_{A}^{*} f_{K}}{\Phi\left(\gamma_{A}^{*}\right)}+2 \delta_{G} \frac{\beta_{0} p_{K} f_{G}}{\Phi\left(\gamma_{A}^{*}\right)}$, then the gas supplier's profit (if going for the nonbinding alternative) is maximized by choosing $\gamma(a)=\gamma_{A}^{*}$, and his corresponding profit is $\bar{C}_{A}$. If $a \in\left(a_{5}, a_{2}\right)$, then the gas supplier's profit is maximized by choosing $\gamma(a)=\hat{\gamma}(a)$, and his corresponding profit is $(\hat{\gamma}(a)-\psi) \cdot G_{A}^{*}(\hat{\gamma}(a))=\frac{(\hat{\gamma}(a)-\psi)\left(\beta_{0} p_{K} f_{G}\right)^{2}}{\Phi(\hat{\gamma}(a))^{2}}=\hat{\Gamma}_{A}(a)>0$ (cf. Appendix C). Note that the latter curve is a concave quadratic function of $a$, which obtains its maximum $\bar{C}_{A}$ at $a_{2}$ and equals $C_{B}\left(a_{4}\right)$ at $a_{4} \stackrel{\text { def }}{=} 2 \delta_{K} \frac{\beta_{0} \gamma_{B}^{*} f_{K}}{\Phi\left(\gamma_{B}^{*}\right)}+2 \delta_{G} \frac{\beta_{0} p_{K} f_{G}}{\Phi\left(\gamma_{B}^{*}\right)}$.

If going for the binding permits level alternative, profit is maximized by choosing $\gamma(a) \in$ $\arg \max _{\gamma \in[\psi, \infty)}(\gamma-\psi) \cdot G_{B}^{*}(\gamma)$ subject to $2 \delta_{K} \sqrt{K_{A}^{*}}+2 \delta_{G} \sqrt{G_{A}^{*}}>a$, i.e., subject to the permits level being binding. First, note that the inequality constraint is satisfied for all $\gamma \geq 0$ if $a \leq a_{5}$. If $a>a_{5}$, then the constraint cannot be satisfied for $\gamma \geq 0$ if $a \geq a_{0} \stackrel{\text { def }}{=} \frac{\beta_{0} \delta_{G}}{f_{G}}$, and the constraint is equivalent to $\gamma<\hat{\gamma}(a)(>0)$ if $a<a_{0}$. In the latter case, i.e., $a \in\left(a_{5}, a_{0}\right)$, the interval of available gas prices is bounded from above by a decreasing function of permits level $a$ (cf. Appendix C), that allows the gas supplier to choose (gas price $\psi$ exactly on $a<a_{1} \stackrel{\text { def }}{=} 2 \delta_{K} \frac{\beta_{0} \psi f_{K}}{\Phi}+2 \delta_{G} \frac{\beta_{0} p_{K} f_{G}}{\Phi}$, and) gas price $\gamma_{B}^{*}$ exactly on $a<a_{4}$. If $a \in\left(a_{-1}, a_{4}\right)$, then the gas supplier's profit (if going for the binding alternative) is maximized over all alternatives with a well-defined outcome (i.e., over all $\gamma>\frac{2(-D)\left(\beta_{0} \delta_{G}-f_{G} a\right)}{\beta_{1} \delta_{K} a}, \gamma \geq \psi$ ) by choosing $\gamma(a)=\gamma_{B}^{*}$, and his corresponding profit is $C_{B}(a)$. If $a \in\left[a_{4}, a_{0}\right)$, his achievable 
profit is lower than $(\hat{\gamma}(a)-\psi) \cdot G_{B}^{*}(\hat{\gamma}(a))=\frac{(\hat{\gamma}(a)-\psi)\left[2 \beta_{0} \delta_{K}(-D)+a Q\right]^{2}}{4 \Psi(\hat{\gamma}(a))^{2}}$, which equals $\hat{\Gamma}_{A}(a)$ (cf. Appendix C). If $a \leq a_{-1}$, then the gas supplier's profit (if going for the binding alternative) cannot be maximized over all alternatives with a well-defined outcome (i.e., over all $\left.\gamma>\frac{2(-D)\left(\beta_{0} \delta_{G}-f_{G} a\right)}{\beta_{1} \delta_{K} a}(>\psi)\right)$.

Since $\hat{\Gamma}_{A}(a)$ is below $C_{B}(a)$ on $a<a_{4}$ and below $\bar{C}_{A}$ on $a>a_{2}$, it follows that the gas supplier goes for the binding permits level alternative if $a \in\left(a_{-1}, a_{4}\right)$ and for the nonbinding alternative if $a \geq a_{4}$.

e) The calculation of $\hat{W}^{M}(a)$ is standard (cf Appendix C).

$f)$ The calculation of $W_{\gamma}(a)$ is standard (cf. our calculations for Lemma 2.1f). $W_{\gamma}(a)$ obtains its maximum at $a^{*}(\gamma)$ as defined in the proof of Lemma 2.1f, and it is easy to verify that $a^{*}\left(\gamma_{B}^{*}\right)>\frac{2 \beta_{0} \delta_{G}(-D)}{R\left(\gamma_{B}^{*}\right)}>0, a^{*}(\psi)>\frac{2 \beta_{0} \delta_{G}(-D)}{R}>0$, and $a^{*}(\psi)>a^{*}\left(\gamma_{B}^{*}\right)$ (cf. our calculations for Lemma $2.1 \mathrm{f}$ and note that $q<\bar{q}$ implies $\left.\beta_{0} f_{G} p_{K}-q Q>0\right)$. Given our previous results for the two scenarios, since $a^{*}\left(\gamma_{B}^{*}\right)<a_{4}$ and $a^{*}(\psi)<a_{1}$, the welfare-maximizing permits level is $a^{*}\left(\gamma_{B}^{*}\right)=a_{M}^{*}$ in the monopolistic and $a^{*}(\psi)=a_{C}^{*}$ in the competitive scenario.

All welfare implications follow immediately from the representation of $W_{\gamma}(a)$. The calculations for the gas supplier's profits are standard (cf. our calculations for Lemma 2.1f).

g) The relation $W_{B}^{C}(q)>W_{A}^{C}(q)$ is easy to verify (cf. our calculations for Lemma $2.1 \mathrm{~g}$ ).

h) To see that $\hat{W}_{A}^{M}(q)<W_{B}^{M}(q)$, note that, for $a=a_{4}$ and $\gamma=\hat{\gamma}\left(a_{4}\right)=\gamma_{B}^{*}$, we have that $K_{A}^{*}=K_{B}^{*}$ and $G_{A}^{*}=G_{B}^{*}$. Therefore, $\hat{W}_{A}^{M}(q) \stackrel{\text { def }}{=} \hat{W}^{M}\left(a_{4}\right)=W_{\gamma_{B}^{*}}\left(a_{4}\right)$, where $\hat{W}^{M}(a)$ and $W_{\gamma}(a)$ are as defined in part e) and f), respectively. Since $a_{M}^{*}(q)=\arg \max _{a} W_{\gamma_{B}^{*}}(a)$ and $a_{M}^{*}(q) \in\left(a_{-1}, a_{4}\right), \hat{W}_{A}^{M}(q)$ must necessarily be lower than $W_{B}^{M}(q) \stackrel{\text { def }}{=} W_{\gamma_{B}^{*}}\left(a_{M}^{*}(q)\right)$.

The relationship between $W_{B}^{M}(q)$ and $W_{A}^{M}(q)$ on the one hand and $q$ and $\tilde{q}(Q)$ on the other hand is easy to verify (cf. Appendix C).

Lemma 2.3 (Necessary Conditions for Equilibria in the Emission Permits Regime if $Q=0$ )

In any equilibrium of the emission permits regime in case of $Q=0$,

a) factor inputs $K$ and $G$ equal $K_{A}^{*}$ and $G_{A}^{*}$ as specified in Lemma 2.1a, respectively, if the emission permits level is non-binding, and

b) factor inputs $K$ and $G$ equal $K_{B}^{*}$ and $G_{B}^{*}$ as specified in Lemma 2.1b, respectively, if the emission permits level is binding and $(\gamma, a)$ satisfies $2 \beta_{0} \delta_{G} D+a R(\gamma)>0$.

c) In the monopolistic scenario, if $a \geq a_{0} \stackrel{\text { def }}{=} \frac{\beta_{0} \delta_{G}}{f_{G}}\left(=a_{1}=a_{2}=a_{4}=a_{5}\right)$, then the permits level is non-binding and gas price $\gamma$ equals $\gamma_{A}^{*} \stackrel{\text { def }}{=} \frac{\Phi}{\beta_{1} p_{K}+2 f_{K}^{2}}+\psi$. If $a \in\left(a_{-1} \stackrel{\text { def }}{=} \frac{2 \beta_{0} \delta_{G}(-D)}{R\left(\gamma_{B}^{*}\right)}, a_{0}\right)$, then the permits level is binding and gas price $\gamma$ equals $\gamma_{B}^{*} \stackrel{\text { def }}{=} \frac{\Psi}{\beta_{1} \delta_{K}^{2}}+\psi$. In either case, the gas supplier's profits are $\Gamma_{A}$ as specified in Lemma 2.1c.

d) In the competitive scenario, where gas price $\gamma$ equals $\psi$, (it is obvious that) permits level a is non-binding if $a \geq a_{1} \stackrel{\text { def }}{=} 2 \delta_{K} \frac{\beta_{0} \psi f_{K}}{\Phi}+2 \delta_{G} \frac{\beta_{0} p_{K} f_{G}}{\Phi}\left(=a_{0}\right)$ and binding if $a \in\left(\frac{2 \beta_{0} \delta_{G}(-D)}{R}, a_{1}\right)$.

e) If the emission permits level is non-binding, then (it is obvious that) the welfare levels equal those of the taxes regime for tax level $p=0$, i.e., $W_{A}^{C}(q)=W_{p=0}^{C}(q)$ and $W_{A}^{M}(q)=W_{p=0}^{M}(q)$ in the competitive and monopolistic scenario, respectively (cf. Lemma 1). In particular, they are below their respective counterparts in the taxes regime (where $p=p^{*}=q$ ).

$f)$ If the emission permits level is binding, then welfare, whenever $2 \beta_{0} \delta_{G} D+a R(\gamma)>0$, equals $W_{\gamma}(a)$ as specified in Lemma 2.1f. It follows that the regulator's welfare-maximizing permits level choice, if going for the binding permits level alternative, is

$$
\begin{aligned}
& a_{C}^{*}(q) \stackrel{\text { def }}{=} a_{1}-\frac{2 \Psi}{\Phi} q \in\left(\frac{2 \beta_{0} \delta_{G}(-D)}{R}, a_{1}\right) \text { and } a_{M}^{*}(q) \stackrel{\text { def }}{=} a_{4}-\frac{4 \Psi}{\Phi+\frac{\beta_{1} p_{K}+2 f_{K}^{2}}{\beta_{1} \delta_{K}^{2}} \Psi} q \in\left(a_{-1}, a_{0}\left(=a_{4}\right)\right) \\
& \text { in the competitive and monopolistic scenario, respectively. }
\end{aligned}
$$


In particular, the regulator's permits level choice is the same in the two scenarios.

If the regulator goes for the binding permits level alternative, then welfare in the competitive scenario $\left(\gamma=\psi, a=a_{C}^{*}\right)$ equals $W_{B}^{C}(q)$ as specified in Lemma 2.1f. In the monopolistic scenario $\left(\gamma=\gamma_{B}^{*}, a=a_{M}^{*}\right)$, welfare equals $W_{B}^{M}(q)$ as specified in Lemma 2.1f, and the gas supplier's profits equal $\Gamma_{A}=\Gamma_{B}(q)$ as specified in Lemma 2.1f.

g) In the competitive scenario, $W_{B}^{C}(q)>W_{A}^{C}(q)$. Therefore, the regulator's welfare-maximizing permits level choice is $a_{C}^{*}$, the emission permits level is binding, and welfare equals $W_{B}^{C}(q)$.

$h)$ In the monopolistic scenario, $W_{B}^{M}(q)>W_{A}^{M}(q)$. Thus, the regulator's welfare-maximizing permits level choice is $a_{M}^{*}$, the emission permits level is binding, and welfare equals $W_{B}^{M}(q)$.

\section{Proof of Lemma 2.3}

c) First, note that the gas supplier's profits in case of a binding permits level are given by $(\gamma-\psi) \cdot G_{B}^{*}(\gamma)$ as specified in the proof of Lemma 2.1c. This curve (considered as a function of $\gamma$ ) obtains its maximum at $\gamma_{B}^{*}$ and, for $\gamma=\gamma_{B}^{*}$, this curve (considered as a function of $a$ ) is constant in $a$ and equals $\bar{C}_{B}:=\frac{\beta_{0}^{2} D^{2}}{4 \beta_{1} \Psi}$. The gas supplier's profits in case of a non-binding permits level are given by $(\gamma-\psi) \cdot G_{A}^{*}(\gamma)$ as specified in the proof of Lemma 2.1c. This curve (considered as a function of $\gamma$ ) obtains its maximum at $\gamma_{A}^{*}$ and, for $\gamma=\gamma_{A}^{*}$, this curve (considered as a function of $a$ ) is constant in $a$ and equals $\bar{C}_{A} \stackrel{\text { def }}{=} \frac{\Phi}{\left(\beta_{1} p_{K}+2 f_{K}^{2}\right)} \cdot \frac{\left(\beta_{0} p_{K} f_{G}\right)^{2}}{(2 \Phi)^{2}}=\bar{C}_{B}$ (for the latter equality, cf. Appendix C). (Cf. the proof of Lemma 2.1c.)

Since $Q=0$, now $a_{-1}<a_{0}=a_{1}=a_{2}=a_{4}=a_{5}$ (cf. our calculations for Lemma 2.1c).

If going for the binding permits level alternative, profit is maximized by choosing $\gamma(a) \in$ $\arg \max _{\gamma \in[\psi, \infty)}(\gamma-\psi) \cdot G_{B}^{*}(\gamma)$ subject to $2 \delta_{K} \sqrt{K_{A}^{*}}+2 \delta_{G} \sqrt{G_{A}^{*}}>a$ (cf. part a) of Lemma 2.3). First, note that going for the binding permits level alternative requires $a<a_{5} \stackrel{\text { def }}{=} \frac{2 \beta_{0} f_{K} \delta_{K}}{\beta_{1} p_{K}+2 f_{K}^{2}}$, since otherwise the inequality constraint cannot be satisfied for $\gamma \geq 0$ : If $a>a_{5}$, then the inequality constraint is equivalent to $\gamma<\hat{\gamma}(a) \stackrel{\text { def }}{=} \frac{a \cdot 2 f_{G}^{2} p_{K}-2 \beta_{0} f_{G} \delta_{G} p_{K}}{2 \beta_{0} \delta_{K} f_{K}-a \cdot\left(\beta_{1} p_{K}+2 f_{K}^{2}\right)}$, the fraction on the right-hand side being lower than zero (since the numerator is positive and the denominator negative), and if $a=a_{5}$, then the inequality constraint is equivalent to $Q>0$, contradicting $Q=0$. If $a<a_{5}$, then the inequality constraint $2 \delta_{K} \frac{\beta_{0} \gamma f_{K}}{\Phi(\gamma)}+2 \delta_{G} \frac{\beta_{0} p_{K} f_{G}}{\Phi(\gamma)}>a$ is equivalent to $\gamma>\hat{\gamma}(a)$. If $a<a_{4} \stackrel{\text { def }}{=} 2 \delta_{K} \frac{\beta_{0} \gamma_{B}^{*} f_{K}}{\Phi\left(\gamma_{B}^{*}\right)}+2 \delta_{G} \frac{\beta_{0} p_{K} f_{G}}{\Phi\left(\gamma_{B}^{*}\right)}=a_{5}$, and if $a>a_{-1}\left(<a_{5}\right)$, then the gas supplier's profit (if going for the binding alternative) is maximized over all alternatives with a well-defined outcome (i.e., over all $\left.\gamma>\frac{2(-D)\left(\beta_{0} \delta_{G}-f_{G} a\right)}{\beta_{1} \delta_{K} a}, \gamma \geq \psi\right)$ by choosing $\gamma(a)=\gamma_{B}^{*}$, and his corresponding profit is $\bar{C}_{B}$. If $a \leq a_{-1}$, then the gas supplier's profit (if going for the binding alternative) cannot be maximized over all alternatives with a well-defined outcome (i.e., over all $\left.\gamma>\frac{2(-D)\left(\beta_{0} \delta_{G}-f_{G} a\right)}{\beta_{1} \delta_{K} a}(>\psi)\right)$.

If going for the non-binding permits level alternative, profit is maximized by choosing $\gamma(a) \in$ $\arg \max _{\gamma \in[\psi, \infty)}(\gamma-\psi) \cdot G_{A}^{*}(\gamma)$ subject to $2 \delta_{K} \sqrt{K_{A}^{*}}+2 \delta_{G} \sqrt{G_{A}^{*}} \leq a$. The inequality constraint is satisfied for all $\gamma \geq 0$ if $a \geq a_{5}$, and the constraint cannot be satisfied for $\gamma>0$ if $a<a_{5}$. Thus, the gas supplier's profit (if going for the non-binding alternative) is maximized by choosing $\gamma(a)=\gamma_{A}^{*}$ on $a \geq a_{5}$, and his corresponding profit is $\bar{C}_{A}$.

It follows that the gas supplier goes for the binding alternative if $a \in\left(a_{-1}, a_{5}\right)$ and for the non-binding alternative if $a \geq a_{5}$.

f) The calculation of $W_{\gamma}(a)$ is standard (cf. our calculations for Lemma 2.1f). $W_{\gamma}(a)$ obtains its maximum at $a^{*}(\gamma)$ as defined in the proof of Lemma 2.1f, and it is easy to verify that $a^{*}\left(\gamma_{B}^{*}\right)=a^{*}(\psi)>\frac{2 \beta_{0} \delta_{G}(-D)}{R}>\frac{2 \beta_{0} \delta_{G}(-D)}{R\left(\gamma_{B}^{*}\right)}$ (cf. our calculations for Lemma 2.1f). Since $a^{*}\left(\gamma_{B}^{*}\right)<a_{0}\left(=a_{4}\right)$ and $a^{*}(\psi)<a_{1}$, and given our previous results for the two scenarios, the welfare-maximizing permits level equals $a^{*}\left(\gamma_{B}^{*}\right)=a_{M}^{*}$ in the monopolistic and $a^{*}(\psi)=a_{C}^{*}$ 
in the competitive scenario.

All welfare implications follow immediately from the representation of $W_{\gamma}(a)$. Finally, it is easy to verify that $\Gamma_{B}(q)=\Gamma_{A}$ (cf. our notes at the beginning of Appendix C).

g) The relation $W_{B}^{C}(q)>W_{A}^{C}(q)$ is easy to verify (cf. our calculations for Lemma $2.1 \mathrm{~g}$ ).

h) The relation $W_{B}^{M}(q)>W_{A}^{M}(q)$ is easy to verify (cf. our calculations for Lemma $2.1 \mathrm{~h}$ ).

Lemma 2 (Equilibria with a Binding Permits Level)

Summarizing Lemma 2.1 to 2.3, $\left(K_{B}^{*}\left(a_{C}^{*}\right), G_{B}^{*}\left(a_{C}^{*}\right), a_{C}^{*}\right)$ is the unique equilibrium (and one with a binding permits level) in the competitive scenario of the emission permits regime. In the monopolistic scenario, if $q<\tilde{q}(Q)$ (which implies $Q \neq 0$ ), then there does not exist an equilibrium with a binding permits level. If $q \geq \tilde{q}(Q)$, then $\left(K_{B}^{*}\left(\gamma_{B}^{*}, a_{M}^{*}\right), G_{B}^{*}\left(\gamma_{B}^{*}, a_{M}^{*}\right), \gamma_{B}^{*}, a_{M}^{*}\right)$ is the unique equilibrium with a binding permits level; if $q>\tilde{q}(Q)$, it is the unique equilibrium.

Lemma 3 and 4 follow from standard calculations (cf. Appendix C).

Lemma 3 (Differences in Welfare and in the Gas Supplier's Profits across Regimes)

a) In the monopolistic scenario, if $q>\tilde{q}(Q)$, then the difference in welfare between the unique equilibrium of the taxes regime, $\left(K^{*}\left(\gamma^{*}, p^{*}\right), G^{*}\left(\gamma^{*}, p^{*}\right), \gamma^{*}, p^{*}\right)$, and the unique equilibrium of the permits regime, $\left(K_{B}^{*}\left(\gamma_{B}^{*}, a_{M}^{*}\right), G_{B}^{*}\left(\gamma_{B}^{*}, a_{M}^{*}\right), \gamma_{B}^{*}, a_{M}^{*}\right)$, is

$$
W^{M}(q)-W_{B}^{M}(q)=\frac{Q^{2}\left[\beta_{0} p_{K} f_{G}-q Q\right]^{2}}{2 \Phi \cdot\left(\beta_{1} p_{K}+2 f_{K}^{2}\right)\left[\beta_{1} \delta_{K}^{2} \Phi+\left(\beta_{1} p_{K}+2 f_{K}^{2}\right) \Psi\right]} .
$$

In particular, if $Q \neq 0$, then this difference is greater than zero, it is increasing in marginal emission cost $q$ if $Q<0$, and it is decreasing in $q$ if $Q>0$ (note that $\beta_{0} p_{K} f_{G}-q Q>0$, which, if $Q>0$, follows from $q<\bar{q}$ ).

If $q \leq \tilde{q}(Q)$ (which implies $Q \neq 0$ ), then the difference in welfare between the unique equilibrium of the taxes regime and any equilibrium of the permits regime is $W^{M}(q)-W_{A}^{M}(q)=$ $W^{M}(q)-W_{p=0}^{M}(q)>0$ and increases in marginal emission cost $q$ (cf. Lemma 1).

b) If $q \geq \tilde{q}(Q)\left(>q_{3}\right.$ if $\left.Q<0\right)$, then the difference in the resulting gas supplier's profits between the unique equilibrium of the taxes regime, $\left(K^{*}\left(\gamma^{*}, p^{*}\right), G^{*}\left(\gamma^{*}, p^{*}\right), \gamma^{*}, p^{*}\right)$, and the binding permits level equilibrium, $\left(K_{B}^{*}\left(\gamma_{B}^{*}, a_{M}^{*}\right), G_{B}^{*}\left(\gamma_{B}^{*}, a_{M}^{*}\right), \gamma_{B}^{*}, a_{M}^{*}\right)$, is

$$
\Gamma(q)-\Gamma_{B}(q)=\frac{Q^{4}\left[\beta_{0} p_{K} f_{G}-q Q\right]^{2}}{4 \Phi \cdot\left(\beta_{1} p_{K}+2 f_{K}^{2}\right)\left[\beta_{1} \delta_{K}^{2} \Phi+\left(\beta_{1} p_{K}+2 f_{K}^{2}\right) \Psi\right]^{2}} .
$$

In particular, if $Q \neq 0$, then this difference is greater than zero; whereas the gas price is higher in the emission permits regime, the demand for gas is lower: $\gamma^{*}<\gamma_{B}^{*}$ and $G^{*}>G_{B}^{*}$. If $q \leq \tilde{q}(Q)$ (which implies $Q \neq 0$ ), then the difference in the resulting gas supplier's profits between the unique equilibrium of the taxes regime and any non-binding permits level equilibrium of the permits regime is

$$
\Gamma(q)-\Gamma_{A}=\frac{\left[\beta_{0} p_{K} f_{G}-q Q\right]^{2}-\left(\beta_{0} p_{K} f_{G}\right)^{2}}{4 \Phi \cdot\left(\beta_{1} p_{K}+2 f_{K}^{2}\right)}>0 .
$$

Here, the gas price is the same in the two regimes $\left(\gamma^{*}=\gamma_{A}^{*}\right)$, and the demand for gas is lower in the permits regime if $Q<0$ (i.e., $G^{*}>G_{A}^{*}$ ) and higher if $Q>0$ (i.e., $G^{*}<G_{A}^{*}$ ).

c) In the competitive scenario, the difference in welfare between the unique equilibrium of the taxes regime, $\left(K^{*}\left(p^{*}\right), G^{*}\left(p^{*}\right), p^{*}\right)$, and the unique equilibrium of the permits regime, $\left(K_{B}^{*}\left(a_{C}^{*}\right), G_{B}^{*}\left(a_{C}^{*}\right), a_{C}^{*}\right)$, is $W^{C}(q)-W_{B}^{C}(q)=0$.

\section{Lemma 4}

For $\psi$ sufficiently high (in relative terms), $\tilde{q}(Q)$ (and thus also $q_{3}<\tilde{q}(Q)$ in case of $Q<0$ ) is (well) below the boundary $\bar{q}$ for marginal emission cost $q$, i.e., $\tilde{q}(Q)<\frac{\beta_{0} f_{K} \psi}{R}$. 


\section{Calculations}

Note that, in some of the subsequent calculations, we use that

which implies that $\quad\left[\beta_{1} \delta_{K}^{2} \Phi-\left(\beta_{1} p_{K}+2 f_{K}^{2}\right) \Psi\right]=-Q^{2}$,

$$
\Phi-\frac{\beta_{1} p_{K}+2 f_{K}^{2}}{\beta_{1} \delta_{K}^{2}} \Psi=2 f_{G}^{2} p_{K}-\frac{\beta_{1} p_{K}+2 f_{K}^{2}}{\beta_{1} \delta_{K}^{2}}\left(\beta_{1} \delta_{G}^{2} p_{K}+2 D^{2}\right)=\frac{-\left[\beta_{1} p_{K} \delta_{G}+2 f_{K} D\right]^{2}}{\beta_{1} \delta_{K}^{2}}=\frac{-Q^{2}}{\beta_{1} \delta_{K}^{2}},
$$

$$
\begin{aligned}
\Psi-\frac{\beta_{1} \delta_{K}^{2}}{\beta_{1} p_{K}+2 f_{K}^{2}} \Phi & =\frac{Q^{2}}{\beta_{1} p_{K}+2 f_{K}^{2}}, \text { and } \\
{\left[\beta_{1} \delta_{K}^{2} \Phi+\left(\beta_{1} p_{K}+2 f_{K}^{2}\right) \Psi\right]^{2} } & =Q^{4}+4 \Psi \Phi \cdot\left(\beta_{1} p_{K}+2 f_{K}^{2}\right) \beta_{1} \delta_{K}^{2} .
\end{aligned}
$$

\section{C.1 Calculations for Lemma 1c}

$$
\begin{aligned}
& W_{\gamma}(p)=\frac{\beta_{0}}{\beta_{1}}\left[2 f_{K} \sqrt{K^{*}}+2 f_{G} \sqrt{G^{*}}\right]-\frac{1}{2 \beta_{1}}\left[2 f_{K} \sqrt{K^{*}}+2 f_{G} \sqrt{G^{*}}\right]^{2}-\left[p_{K} K^{*}+\gamma G^{*}\right]-q\left[2 \delta_{K} \sqrt{K^{*}}+2 \delta_{G} \sqrt{G^{*}}\right] \\
& =\frac{\beta_{0}}{\beta_{1}} \cdot \frac{\left[2 \beta_{0}\left(f_{K}^{2} \gamma+f_{G}^{2} p_{K}\right)-2 \beta_{1} p\left(f_{K} \delta_{K} \gamma+f_{G} \delta_{G} p_{K}\right)\right]}{\Phi(\gamma)} \\
& -\frac{1}{2 \beta_{1}} \cdot \frac{\left[2 \beta_{0}\left(f_{K}^{2} \gamma+f_{G}^{2} p_{K}\right)-2 \beta_{1} p\left(f_{K} \delta_{K} \gamma+f_{G} \delta_{G} p_{K}\right)\right]^{2}}{\Phi(\gamma)^{2}} \\
& -p_{K} \cdot \frac{\left[\beta_{0} \gamma f_{K}-p R(\gamma)\right]^{2}}{\Phi(\gamma)^{2}}-\gamma \cdot \frac{\left[\beta_{0} p_{K} f_{G}-p Q\right]^{2}}{\Phi(\gamma)^{2}} \\
& -2 q \delta_{K} \cdot \frac{\left[\beta_{0} \gamma f_{K}-p R(\gamma)\right]}{\Phi(\gamma)}-2 q \delta_{G} \cdot \frac{\left[\beta_{0} p_{K} f_{G}-p Q\right]}{\Phi(\gamma)} \text {, i.e. } \\
& 2 \beta_{1} \Phi(\gamma)^{2} \cdot W_{\gamma}(p)=2 \beta_{0}\left[2 \beta_{0}\left(f_{K}^{2} \gamma+f_{G}^{2} p_{K}\right)-2 \beta_{1} p\left(f_{K} \delta_{K} \gamma+f_{G} \delta_{G} p_{K}\right)\right]\left[\beta_{1} p_{K} \gamma+2 f_{K}^{2} \gamma+2 f_{G}^{2} p_{K}\right] \\
& -\left[2 \beta_{0}\left(f_{K}^{2} \gamma+f_{G}^{2} p_{K}\right)-2 \beta_{1} p\left(f_{K} \delta_{K} \gamma+f_{G} \delta_{G} p_{K}\right)\right]^{2} \\
& -2 \beta_{1} p_{K}\left[\beta_{0} \gamma f_{K}+\left(2 f_{G} f_{K} \delta_{G}-\delta_{K} \beta_{1} \gamma-2 \delta_{K} f_{G}^{2}\right) p\right]^{2} \\
& -2 \beta_{1} \gamma\left[\beta_{0} p_{K} f_{G}+\left(2 f_{G} f_{K} \delta_{K}-\delta_{G} \beta_{1} p_{K}-2 \delta_{G} f_{K}^{2}\right) p\right]^{2} \\
& -4 \beta_{1} q \delta_{K}\left[\beta_{0} \gamma f_{K}+2 f_{G} f_{K} \delta_{G} p-\delta_{K} p \beta_{1} \gamma-2 \delta_{K} p f_{G}^{2}\right] \Phi(\gamma) \\
& -4 \beta_{1} q \delta_{G}\left[\beta_{0} p_{K} f_{G}+2 f_{K} f_{G} \delta_{K} p-\delta_{G} p \beta_{1} p_{K}-2 \delta_{G} p f_{K}^{2}\right] \Phi(\gamma) \\
& =\tilde{W}_{2}^{\prime}(\gamma) \cdot p^{2}+\tilde{W}_{1}^{\prime}(\gamma) \cdot p+\tilde{W}_{0}^{\prime}(\gamma), \text { where } \\
& \tilde{W}_{2}^{\prime}(\gamma)=-\left(2 \beta_{1}\right)^{2}\left(f_{K} \delta_{K} \gamma+f_{G} \delta_{G} p_{K}\right)^{2} \\
& -2 \beta_{1} p_{K}\left(2 f_{G} f_{K} \delta_{G}-\delta_{K} \beta_{1} \gamma-\delta_{K} 2 f_{G}^{2}\right)^{2}-2 \beta_{1} \gamma\left(2 f_{G} f_{K} \delta_{K}-\delta_{G} \beta_{1} p_{K}-\delta_{G} 2 f_{K}^{2}\right)^{2} \\
& =-2 \beta_{1} \cdot \Psi(\gamma) \cdot \Phi(\gamma), \\
& \tilde{W}_{1}^{\prime}(\gamma)=-4 \beta_{0} \beta_{1}\left(f_{K} \delta_{K} \gamma+f_{G} \delta_{G} p_{K}\right)\left(\beta_{1} p_{K} \gamma+2 f_{K}^{2} \gamma+2 f_{G}^{2} p_{K}\right) \\
& +4 \beta_{0} \beta_{1}\left(2 f_{K}^{2} \gamma+2 f_{G}^{2} p_{K}\right)\left(f_{K} \delta_{K} \gamma+f_{G} \delta_{G} p_{K}\right) \\
& -4 \beta_{0} \beta_{1} p_{K} f_{K} \gamma\left(2 f_{G} f_{K} \delta_{G}-\delta_{K} \beta_{1} \gamma-\delta_{K} 2 f_{G}^{2}\right)-4 \beta_{0} \beta_{1} p_{K} f_{G} \gamma\left(2 f_{G} f_{K} \delta_{K}-\delta_{G} \beta_{1} p_{K}-\delta_{G} 2 f_{K}^{2}\right) \\
& -4 \beta_{1} q\left(2 f_{G} f_{K} \delta_{G} \delta_{K}-\delta_{K}^{2} \beta_{1} \gamma-\delta_{K}^{2} 2 f_{G}^{2}\right) \Phi(\gamma)-4 \beta_{1} q\left(2 f_{G} f_{K} \delta_{K} \delta_{G}-\delta_{G}^{2} \beta_{1} p_{K}-\delta_{G}^{2} 2 f_{K}^{2}\right) \Phi(\gamma) \\
& =4 \beta_{1} q \Phi(\gamma)\left[\delta_{K}^{2}\left(\beta_{1} \gamma+2 f_{G}^{2}\right)+\delta_{G}^{2}\left(\beta_{1} p_{K}+2 f_{K}^{2}\right)-4 f_{G} f_{K} \delta_{G} \delta_{K}\right] \\
& =4 \beta_{1} q \cdot \Phi(\gamma) \cdot \Psi(\gamma) \text {, and } \\
& \tilde{W}_{0}^{\prime}(\gamma)=\left(2 \beta_{0}\right)^{2}\left(f_{K}^{2} \gamma+f_{G}^{2} p_{K}\right)\left(\beta_{1} p_{K} \gamma+2 f_{K}^{2} \gamma+2 f_{G}^{2} p_{K}\right)-\left(2 \beta_{0}\right)^{2}\left(f_{K}^{2} \gamma+f_{G}^{2} p_{K}\right)^{2} \\
& -2 \beta_{1} p_{K}\left(\beta_{0} \gamma f_{K}\right)^{2}-2 \beta_{1} \gamma\left(\beta_{0} p_{K} f_{G}\right)^{2} \\
& -4 \beta_{1} q \delta_{K} \beta_{0} \gamma f_{K} \Phi(\gamma)-4 \beta_{1} q \delta_{G} \beta_{0} p_{K} f_{G} \Phi(\gamma) \\
& =2 \beta_{0}\left[\beta_{1} p_{K} \gamma+2 f_{K}^{2} \gamma+2 f_{G}^{2} p_{K}\right] \beta_{0}\left(f_{K}^{2} \gamma+f_{G}^{2} p_{K}\right)-2 \beta_{0} \Phi(\gamma) 2 \beta_{1} q\left(f_{K} \delta_{K} \gamma+\delta_{G} f_{G} p_{K}\right) \\
& =2 \beta_{0} \cdot \Phi(\gamma) \cdot\left[\beta_{0}\left(f_{K}^{2} \gamma+f_{G}^{2} p_{K}\right)-2 \beta_{1} q\left(f_{K} \delta_{K} \gamma+\delta_{G} f_{G} p_{K}\right)\right] \text {. }
\end{aligned}
$$

\section{C.2 Calculations for Lemma 2.1c}

To see that $0<a_{-1}<a_{0}<a_{1}<a_{2}<a_{4}<a_{5}<a_{6}$, note that the first inequality is obvious, that $a_{0}>a_{-1}$ is equivalent to $\frac{2 \beta_{0} \delta_{G}(-D)}{R+\frac{\Psi}{\delta_{K}}}<\frac{\beta_{0} \delta_{G}}{f_{G}} \Leftrightarrow \beta_{1} \delta_{K} \psi+\frac{\Psi}{\delta_{K}}>0$, a true statement, that $a_{1}>a_{0}$ is equivalent to

$$
\begin{aligned}
& \frac{\beta_{0} \delta_{G}}{f_{G}}<\frac{2 \beta_{0}\left(f_{K} \delta_{K} \psi+f_{G} \delta_{G} p_{K}\right)}{\Phi} \\
\Leftrightarrow & \delta_{G}\left[\left(\beta_{1} p_{K}+2 f_{K}^{2}\right) \psi+2 f_{G}^{2} p_{K}\right]<2 f_{G}\left(f_{K} \delta_{K} \psi+f_{G} \delta_{G} p_{K}\right) \\
\Leftrightarrow \quad & Q<0
\end{aligned}
$$


that $a_{2}>a_{1}$ is equivalent to

$$
\begin{array}{ll} 
& \frac{\beta_{0}}{\Phi}\left[f_{K} \delta_{K} \psi+f_{G} \delta_{G} p_{K}+\frac{f_{K} \delta_{K}}{\beta_{1} p_{K}+2 f_{K}^{2}} \Phi\right]>\frac{2 \beta_{0}\left(f_{K} \delta_{K} \psi+f_{G} \delta_{G} p_{K}\right)}{\Phi} \\
\Leftrightarrow & \left(f_{K} \delta_{K} \psi+f_{G} \delta_{G} p_{K}\right)+\frac{f_{K} \delta_{K}}{\beta_{1} p_{K}+2 f_{K}^{2}} \Phi>2\left(f_{K} \delta_{K} \psi+f_{G} \delta_{G} p_{K}\right) \\
\Leftrightarrow & -\left(\beta_{1} p_{K}+2 f_{K}^{2}\right)\left(f_{K} \delta_{K} \psi+f_{G} \delta_{G} p_{K}\right)+f_{K} \delta_{K} \Phi>0 \\
\Leftrightarrow & -\left(\beta_{1} p_{K}+2 f_{K}^{2}\right) f_{G} \delta_{G} p_{K}+f_{K} \delta_{K} 2 f_{G}^{2} p_{K}>0 \\
\Leftrightarrow & -f_{G} p_{K}\left[\beta_{1} \delta_{G} p_{K}+2 f_{K}\left(f_{K} \delta_{G}-f_{G} \delta_{K}\right)\right]>0 \\
\Leftrightarrow & -Q>0,
\end{array}
$$

that $a_{4}>a_{2}$ is equivalent to

$$
\begin{aligned}
& \frac{2 \beta_{0}\left(f_{K} \delta_{K} \psi+f_{G} \delta_{G} p_{K}\right)+2 \frac{\beta_{0} f_{K} \delta_{K}}{\beta_{1} \delta_{K}^{2}} \Psi}{\Phi+\frac{\beta_{1} p_{K}+2 f_{K}^{2}}{\beta_{1} \delta_{K}^{2}} \Psi}>\frac{\beta_{0}}{\Phi}\left[f_{K} \delta_{K} \psi+f_{G} \delta_{G} p_{K}+\frac{f_{K} \delta_{K}}{\beta_{1} p_{K}+2 f_{K}^{2}} \Phi\right] \\
\Leftrightarrow & \left(f_{K} \delta_{K} \psi+f_{G} \delta_{G} p_{K}\right) \Phi+\frac{f_{K} \delta_{K}}{\beta_{1} \delta_{K}^{2}} \Psi \Phi>\frac{f_{K} \delta_{K}}{\beta_{1} p_{K}+2 f_{K}^{2}} \Phi^{2}+\left(f_{K} \delta_{K} \psi+f_{G} \delta_{G} p_{K}\right) \frac{\beta_{1} p_{K}+2 f_{K}^{2}}{\beta_{1} \delta_{K}^{2}} \Psi \\
\Leftrightarrow & \left(f_{K} \delta_{K} \psi+f_{G} \delta_{G} p_{K}\right)\left[\Phi-\frac{\beta_{1} p_{K}+2 f_{K}^{2}}{\beta_{1} \delta_{K}^{2}} \Psi\right]+\frac{f_{K} \delta_{K} \Phi}{\beta_{1} p_{K}+2 f_{K}^{2}}\left[\frac{\beta_{1} p_{K}+2 f_{K}^{2}}{\beta_{1} \delta_{K}^{2}} \Psi-\Phi\right]>0 \\
\Leftrightarrow & \left(f_{K} \delta_{K} \psi+f_{G} \delta_{G} p_{K}\right) \frac{-Q^{2}}{\beta_{1} \delta_{K}^{2}}+\frac{f_{K} \delta_{K} \Phi}{\beta_{1} p_{K}+2 f_{K}^{2}} \cdot \frac{Q^{2}}{\beta_{1} \delta_{K}^{2}}>0 \\
\Leftrightarrow \quad & -\left(\beta_{1} p_{K}+2 f_{K}^{2}\right)\left(f_{K} \delta_{K} \psi+f_{G} \delta_{G} p_{K}\right)+f_{K} \delta_{K} \Phi>0 \\
\Leftrightarrow \quad & -Q>0,
\end{aligned}
$$

that $a_{4}<a_{5}$ is equivalent to

$$
\frac{2 \beta_{0}\left(f_{K} \delta_{K} \psi+f_{G} \delta_{G} p_{K}\right)+2 \frac{\beta_{0} f_{K}}{\beta_{1} \delta_{K}} \Psi}{\Phi+\frac{\beta_{1} p_{K}+2 f_{K}^{2}}{\beta_{1} \delta_{K}^{2}} \Psi}<\frac{2 \beta_{0} \delta_{K} f_{K}}{\beta_{1} p_{K}+2 f_{K}^{2}}
$$

$\Leftrightarrow \quad \delta_{K} f_{K} \Phi+\frac{\beta_{1} p_{K}+2 f_{K}^{2}}{\beta_{1} \delta_{K}^{2}} \delta_{K} f_{K} \Psi>\left(\beta_{1} p_{K}+2 f_{K}^{2}\right)\left(f_{K} \delta_{K} \psi+f_{G} \delta_{G} p_{K}\right)+\left(\beta_{1} p_{K}+2 f_{K}^{2}\right) \frac{f_{K} \delta_{K}}{\beta_{1} \delta_{K}^{2}} \Psi$

$\Leftrightarrow \quad \delta_{K} f_{K} \cdot\left[\left(\beta_{1} p_{K}+2 f_{K}^{2}\right) \psi+2 f_{G}^{2} p_{K}\right]>\left(\beta_{1} p_{K}+2 f_{K}^{2}\right)\left(f_{K} \delta_{K} \psi+f_{G} \delta_{G} p_{K}\right)$

$\Leftrightarrow 2 f_{G}^{2} p_{K} \delta_{K} f_{K}>\left(\beta_{1} p_{K}+2 f_{K}^{2}\right) f_{G} \delta_{G} p_{K}$

$\Leftrightarrow \quad Q<0$,

and that $a_{5}<a_{6}$ is equivalent to

$$
\begin{aligned}
& \frac{2 \beta_{0} \delta_{K} f_{K}}{\beta_{1} p_{K}+2 f_{K}^{2}}<\frac{2 \beta_{0} \delta_{K} D}{\beta_{1} \delta_{G} p_{K}+2 f_{K} D} \\
\Leftrightarrow & f_{K} \cdot\left[\beta_{1} \delta_{G} p_{K}+2 f_{K} D\right]>\left(\beta_{1} p_{K}+2 f_{K}^{2}\right) \cdot D \\
\Leftrightarrow & \delta_{G} f_{K}>D,
\end{aligned}
$$

a true statement. To see that $a_{3} \in\left(a_{2}, a_{4}\right)$, note that $a_{3}>a_{2}$ is equivalent to

$$
\begin{aligned}
& \frac{-2 \beta_{0} \delta_{K} D}{-Q}-\frac{2 \beta_{0} p_{K} f_{G}}{-Q} \sqrt{\frac{\Psi}{\Phi} \cdot \frac{\beta_{1} \delta_{K}^{2}}{\left(\beta_{1} p_{K}+2 f_{K}^{2}\right)}}>\frac{\beta_{0}\left(p_{K} f_{G} Q+\delta_{K} f_{K} 2 \Phi\right)}{\left(\beta_{1} p_{K}+2 f_{K}^{2}\right) \Phi} \\
& \Leftrightarrow 2 p_{K} f_{G}\left(\beta_{1} p_{K}+2 f_{K}^{2}\right) \Phi \sqrt{\frac{\Psi}{\Phi} \cdot \frac{\beta_{1} \delta_{K}^{2}}{\left(\beta_{1} p_{K}+2 f_{K}^{2}\right)}}+2 \delta_{K} D\left(\beta_{1} p_{K}+2 f_{K}^{2}\right) \Phi<\left(p_{K} f_{G} Q+\delta_{K} f_{K} 2 \Phi\right) Q \\
& \Leftrightarrow 2 p_{K} f_{G}\left(\beta_{1} p_{K}+2 f_{K}^{2}\right) \Phi \sqrt{\frac{\Psi}{\Phi} \cdot \frac{\beta_{1} \delta_{K}^{2}}{\left(\beta_{1} p_{K}+2 f_{K}^{2}\right)}}+2 \delta_{K} D \beta_{1} p_{K} \Phi \\
& <\left(p_{K} f_{G} Q+\delta_{K} f_{K} 2 \Phi\right) \beta_{1} \delta_{G} p_{K}+p_{K} f_{G} Q 2 f_{K} D \\
& \Leftrightarrow 2 p_{K} f_{G}\left(\beta_{1} p_{K}+2 f_{K}^{2}\right) \Phi \sqrt{\frac{\Psi}{\Phi} \cdot \frac{\beta_{1} \delta_{K}^{2}}{\left(\beta_{1} p_{K}+2 f_{K}^{2}\right)}}+2 \beta_{1} p_{K} \Phi \delta_{K}\left(D-f_{K} \delta_{G}\right)<p_{K} f_{G} Q^{2} \\
& \Leftrightarrow 2\left(\beta_{1} p_{K}+2 f_{K}^{2}\right) \Phi \sqrt{\frac{\Psi}{\Phi} \cdot \frac{\beta_{1} \delta_{K}^{2}}{\left(\beta_{1} p_{K}+2 f_{K}^{2}\right)}}-2 \beta_{1} \Phi \delta_{K}^{2}-Q^{2}<0 \\
& \Leftrightarrow \sqrt{4 \Phi \Psi\left(\beta_{1} p_{K}+2 f_{K}^{2}\right) \beta_{1} \delta_{K}^{2}}<2 \beta_{1} \Phi \delta_{K}^{2}-\left[\beta_{1} \delta_{K}^{2} \Phi-\left(\beta_{1} p_{K}+2 f_{K}^{2}\right) \Psi\right] \quad\left(=\beta_{1} \delta_{K}^{2} \Phi+\left(\beta_{1} p_{K}+2 f_{K}^{2}\right) \Psi\right) \\
& \Leftrightarrow 4 \Phi \Psi\left(\beta_{1} p_{K}+2 f_{K}^{2}\right) \beta_{1} \delta_{K}^{2}<\left[\beta_{1} \delta_{K}^{2} \Phi+\left(\beta_{1} p_{K}+2 f_{K}^{2}\right) \Psi\right]^{2} \\
& \Leftrightarrow \quad 0<\left[\beta_{1} \delta_{K}^{2} \Phi-\left(\beta_{1} p_{K}+2 f_{K}^{2}\right) \Psi\right]^{2},
\end{aligned}
$$

a true statement, and that, in Lemma 2.1f, we show that $a_{3}=a_{4}-4 \Psi \cdot\left[\Phi+\frac{\beta_{1} p_{K}+2 f_{K}^{2}}{\beta_{1} \delta_{K}^{2}} \Psi\right]^{-1} \cdot q_{3}$ with $q_{3}>0$. 
The intersections of $C_{B}(a)$ with $\bar{C}_{A}$ are determined by

$$
\begin{aligned}
& \frac{\Phi}{\left(\beta_{1} p_{K}+2 f_{K}^{2}\right)} \cdot \frac{\left(\beta_{0} p_{K} f_{G}\right)^{2}}{(2 \Phi)^{2}}=\frac{\Psi\left[2 \beta_{0} \delta_{K}(-D)+a Q\right]^{2}}{\beta_{1} \delta_{K}^{2} 4(2 \Psi)^{2}} \\
& \Leftrightarrow \quad\left[2 \beta_{0} \delta_{K}(-D)+a Q\right]^{2}=\frac{\Phi\left(\beta_{0} p_{K} f_{G}\right)^{2} \beta_{1} \delta_{K}^{2} 4(2 \Psi)^{2}}{\left(\beta_{1} p_{K}+2 f_{K}^{2}\right)(2 \Phi)^{2} \Psi} \\
& \Leftrightarrow 2 \beta_{0} \delta_{K}(-D)+a Q= \pm \sqrt{\frac{\Phi\left(\beta_{0} p_{K} f_{G}\right)^{2} \beta_{1} \delta_{K}^{2} 4(2 \Psi)^{2}}{\left(\beta_{1} p_{K}+2 f_{K}^{2}\right)(2 \Phi)^{2} \Psi}} \quad\left(= \pm \sqrt{\frac{\Phi \beta_{1} \delta_{K}^{2}}{\Psi\left(\beta_{1} p_{K}+2 f_{K}^{2}\right)}} \cdot \frac{\beta_{0} p_{K} f_{G} 2 \Psi}{\Phi}\right) \\
& \Leftrightarrow \quad a Q= \pm \sqrt{\frac{\Phi \beta_{1} \delta_{K}^{2}}{\Psi\left(\beta_{1} p_{K}+2 f_{K}^{2}\right)}} \cdot \frac{\beta_{0} p_{K} f_{G} 2 \Psi}{\Phi}+2 \beta_{0} \delta_{K} D \\
& \Leftrightarrow \quad a=\frac{2 \beta_{0} \delta_{K} D}{Q}+\frac{2 \beta_{0} p_{K} f_{G}}{-Q} \cdot \frac{\Psi}{\Phi} \sqrt{\frac{\Phi \beta_{1} \delta_{K}^{2}}{\Psi\left(\beta_{1} p_{K}+2 f_{K}^{2}\right)}} .
\end{aligned}
$$

\section{C.3 Calculations for Lemma 2.1d}

Note that $\frac{2 \beta_{0} \delta_{G}(-D)}{R}<a_{1}$ is equivalent to (the true statement)

$$
\begin{aligned}
& \frac{2 \beta_{0} \delta_{G}(-D)}{R}<\frac{2 \beta_{0}\left(f_{K} \delta_{K} \psi+f_{G} \delta_{G} p_{K}\right)}{\Phi} \\
\Leftrightarrow & \delta_{G}(-D)\left[\left(\beta_{1} p_{K}+2 f_{K}^{2}\right) \psi+2 f_{G}^{2} p_{K}\right]<\left(f_{K} \delta_{K} \psi+f_{G} \delta_{G} p_{K}\right)\left[\beta_{1} \delta_{K} \psi+2 f_{G}(-D)\right] \\
\Leftrightarrow & \beta_{1} \psi f_{K}\left(-p_{K} \delta_{G}^{2}-\psi \delta_{K}^{2}\right)+2 f_{K}(-D) \psi\left(f_{K} \delta_{G}-f_{G} \delta_{K}\right)<0 \\
\Leftrightarrow & \Psi>0 .
\end{aligned}
$$

\section{C.4 Calculations for Lemma 2.1f}

$$
\begin{aligned}
& W_{\gamma}(a)=\frac{\beta_{0}}{\beta_{1}}\left[2 f_{K} \sqrt{K^{*}}+2 f_{G} \sqrt{G^{*}}\right]-\frac{1}{2 \beta_{1}}\left[2 f_{K} \sqrt{K^{*}}+2 f_{G} \sqrt{G^{*}}\right]^{2}-\left[p_{K} K^{*}+\gamma G^{*}\right]-q \cdot a \\
& =\frac{\beta_{0}}{\beta_{1}} \cdot \frac{\left[2 \beta_{0} D^{2}+\beta_{1} a\left(f_{K} \delta_{K} \gamma+f_{G} \delta_{G} p_{K}\right)\right]}{\Psi(\gamma)}-\frac{1}{2 \beta_{1}} \cdot \frac{\left[2 \beta_{0} D^{2}+\beta_{1} a\left(f_{K} \delta_{K} \gamma+f_{G} \delta_{G} p_{K}\right)\right]^{2}}{\Psi(\gamma)^{2}} \\
& -p_{K} \frac{\left[2 \beta_{0} \delta_{G} D+\left[\delta_{K} \beta_{1} \gamma-2 f_{G}\left(\delta_{G} f_{K}-\delta_{K} f_{G}\right)\right] a\right]^{2}}{4 \Psi(\gamma)^{2}} \\
& -\gamma \frac{\left[2 \beta_{0} \delta_{K}(-D)+\left[\delta_{G} \beta_{1} p_{K}-2 f_{K}\left(\delta_{K} f_{G}-\delta_{G} f_{K}\right)\right] a\right]^{2}}{4 \Psi(\gamma)^{2}} \\
& -q \cdot a \\
& =\frac{1}{4 \beta_{1} \Psi(\gamma)^{2}} \cdot\left[4 \beta_{0}\left[2 \beta_{0} D^{2}+\beta_{1} a\left(f_{K} \delta_{K} \gamma+f_{G} \delta_{G} p_{K}\right)\right]\left[\beta_{1}\left(\delta_{K}^{2} \gamma+\delta_{G}^{2} p_{K}\right)+2 D^{2}\right]\right. \\
& -2\left[2 \beta_{0} D^{2}+\beta_{1} a\left(f_{K} \delta_{K} \gamma+f_{G} \delta_{G} p_{K}\right)\right]^{2} \\
& -p_{K} \beta_{1}\left[2 \beta_{0} \delta_{G} D+\left[\delta_{K} \beta_{1} \gamma-2 f_{G} D\right] a\right]^{2} \\
& \left.-\gamma \beta_{1}\left[2 \beta_{0} \delta_{K}(-D)+\left[\delta_{G} \beta_{1} p_{K}-2 f_{K}(-D)\right] a\right]^{2}-q \cdot a \cdot 4 \beta_{1} \Psi(\gamma)^{2} \quad\right] \\
& =\frac{\tilde{V}_{2}^{\prime}(\gamma) \cdot a^{2}+\tilde{V}_{1}^{\prime}(\gamma) \cdot a+\tilde{V}_{0}^{\prime}(\gamma)}{4 \beta_{1} \Psi(\gamma)^{2}} \text {, where } \\
& \tilde{V}_{2}^{\prime}(\gamma)=-2 \beta_{1}^{2}\left(f_{K} \delta_{K} \gamma+f_{G} \delta_{G} p_{K}\right)^{2}-p_{K} \beta_{1}\left[\delta_{K} \beta_{1} \gamma-2 f_{G} D\right]^{2}-\gamma \beta_{1}\left[\delta_{G} \beta_{1} p_{K}-2 f_{K}(-D)\right]^{2} \\
& =-\beta_{1} \cdot \Psi(\gamma) \cdot \Phi(\gamma) \text {, } \\
& \tilde{V}_{1}^{\prime}(\gamma)=4 \beta_{0} \beta_{1}\left(f_{K} \delta_{K} \gamma+f_{G} \delta_{G} p_{K}\right) \Psi(\gamma) \\
& -4 \cdot 2 \beta_{0} \beta_{1}\left(f_{K} \delta_{G}-f_{G} \delta_{K}\right)^{2}\left(f_{K} \delta_{K} \gamma+f_{G} \delta_{G} p_{K}\right) \\
& -p_{K} 4 \beta_{0} \beta_{1} \delta_{G}\left(\delta_{G} f_{K}-\delta_{K} f_{G}\right)\left[\delta_{K} \beta_{1} \gamma-2 f_{G}\left(\delta_{G} f_{K}-\delta_{K} f_{G}\right)\right] \\
& -\gamma 4 \beta_{0} \beta_{1} \delta_{K}\left(\delta_{K} f_{G}-\delta_{G} f_{K}\right)\left[\delta_{G} \beta_{1} p_{K}-2 f_{K}\left(\delta_{K} f_{G}-\delta_{G} f_{K}\right)\right]-q 4 \beta_{1} \Psi(\gamma)^{2} \\
& =4 \beta_{0} \beta_{1}\left(f_{K} \delta_{K} \gamma+f_{G} \delta_{G} p_{K}\right) \Psi(\gamma)-4 \beta_{1} q \Psi(\gamma)^{2} \text {, and } \\
& \tilde{V}_{0}^{\prime}(\gamma)=4 \beta_{0} 2 \beta_{0} D^{2}\left[\beta_{1}\left(\delta_{K}^{2} \gamma+\delta_{G}^{2} p_{K}\right)+2 D^{2}\right]-2\left(2 \beta_{0}\right)^{2}\left(f_{K} \delta_{G}-f_{G} \delta_{K}\right)^{2} D^{2} \\
& -p_{K} \beta_{1}\left(2 \beta_{0}\right)^{2} \delta_{G}^{2} D^{2}-\gamma \beta_{1}\left(2 \beta_{0}\right)^{2} \delta_{K}^{2} D^{2} \\
& =4 \beta_{0}^{2} D^{2}\left[\beta_{1}\left(\delta_{K}^{2} \gamma+\delta_{G}^{2} p_{K}\right)+2\left(f_{K} \delta_{G}-f_{G} \delta_{K}\right)^{2}\right] \\
& =4 \beta_{0}^{2} D^{2} \Psi(\gamma) \text {. }
\end{aligned}
$$


Note that $a^{*}(\psi)<a^{*}\left(\gamma_{B}^{*}\right)$ is equivalent to

$$
\begin{aligned}
& \beta_{0}\left(f_{K} \delta_{K} \psi+f_{G} \delta_{G} p_{K}\right)\left[\Phi+\left(\beta_{1} p_{K}+2 f_{K}^{2}\right) \frac{\Psi}{\beta_{1} \delta_{K}^{2}}\right]-q \Psi \cdot\left[\Phi+\left(\beta_{1} p_{K}+2 f_{K}^{2}\right) \frac{\Psi}{\beta_{1} \delta_{K}^{2}}\right] \\
& <\beta_{0}\left(f_{K} \delta_{K} \psi+f_{G} \delta_{G} p_{K}\right) \Phi+\frac{\beta_{0} f_{K} \delta_{K}}{\beta_{1} \delta_{K}^{2}} \Psi \Phi-2 q \Psi \Phi \\
& \Leftrightarrow \quad \beta_{0}\left(f_{K} \delta_{K} \psi+f_{G} \delta_{G} p_{K}\right)\left(\beta_{1} p_{K}+2 f_{K}^{2}\right) \frac{\Psi}{\beta_{1} \delta_{K}^{2}}-q \frac{\Psi^{2}}{\beta_{1} \delta_{K}^{2}}\left(\beta_{1} p_{K}+2 f_{K}^{2}\right)<\frac{\beta_{0} f_{K} \delta_{K}}{\beta_{1} \delta_{K}^{2}} \Psi \Phi-q \Psi \Phi \\
& \Leftrightarrow \quad \beta_{0}\left(f_{K} \delta_{K} \psi+f_{G} \delta_{G} p_{K}\right)\left(\beta_{1} p_{K}+2 f_{K}^{2}\right)-q \Psi\left(\beta_{1} p_{K}+2 f_{K}^{2}\right) \\
& <\beta_{0} f_{K} \delta_{K}\left[2 f_{G}^{2} p_{K}+\left(\beta_{1} p_{K}+2 f_{K}^{2}\right) \psi\right]-q \beta_{1} \delta_{K}^{2} \Phi \\
& \Leftrightarrow \quad \beta_{0}\left(f_{K} \delta_{K} \psi+f_{G} \delta_{G} p_{K}\right)\left(\beta_{1} p_{K}+2 f_{K}^{2}\right)-\beta_{0} f_{K} \delta_{K} 2 f_{G}^{2} p_{K}-\beta_{0} f_{K} \delta_{K}\left(\beta_{1} p_{K}+2 f_{K}^{2}\right) \psi \\
& <q\left[\left(\beta_{1} p_{K}+2 f_{K}^{2}\right) \Psi-\beta_{1} \delta_{K}^{2} \Phi\right] \\
& \Leftrightarrow \quad \beta_{0} f_{G} p_{K}\left[\beta_{1} \delta_{G} p_{K}+2 f_{K} D\right]<q Q^{2} \\
& \Leftrightarrow \quad \beta_{0} f_{G} p_{K} Q<q Q^{2} \text {. }
\end{aligned}
$$

To see that $a^{*}(\psi)>\frac{2 \beta_{0} \delta_{G}(-D)}{R}$, note that $2 \beta_{0} \delta_{G} D+a^{*}(\psi) R>0$ is equivalent to (the true statement)

$$
\begin{array}{lll} 
& 2 \beta_{0} \delta_{G}\left(\delta_{G} f_{K}-\delta_{K} f_{G}\right)+\left[\beta_{1} \delta_{K} \psi-2 f_{G}\left(\delta_{G} f_{K}-\delta_{K} f_{G}\right)\right] \cdot a^{*}(\psi)>0 \\
\Leftrightarrow & 2 \beta_{0} \delta_{G}\left(\delta_{G} f_{K}-\delta_{K} f_{G}\right)+\left[\beta_{1} \delta_{K} \psi-2 f_{G}\left(\delta_{G} f_{K}-\delta_{K} f_{G}\right)\right] \cdot 2 \frac{\beta_{0}\left(f_{K} \delta_{K} \psi+f_{G} \delta_{G} p_{K}\right)-q \Psi}{\Phi}>0 \\
\Leftrightarrow & \beta_{0}\left[\delta_{G}\left(\delta_{G} f_{K}-\delta_{K} f_{G}\right) \Phi+\left[\beta_{1} \delta_{K} \psi-2 f_{G}\left(\delta_{G} f_{K}-\delta_{K} f_{G}\right)\right]\left(f_{K} \delta_{K} \psi+f_{G} \delta_{G} p_{K}\right)\right] \\
& >q \Psi \cdot\left[\beta_{1} \delta_{K} \psi-2 f_{G}\left(\delta_{G} f_{K}-\delta_{K} f_{G}\right)\right] \\
\Leftrightarrow & \beta_{0} f_{K} \psi\left[\beta_{1}\left(\delta_{K}^{2} \psi+\delta_{G}^{2} p_{K}\right)+2\left(f_{K} \delta_{G}-f_{G} \delta_{K}\right)^{2}\right]>q \Psi R \\
\Leftrightarrow & q<\frac{\beta_{0} f_{K} \psi}{R}(=\bar{q}) .
\end{array}
$$

Furthermore, note that $a^{*}\left(\gamma_{B}^{*}\right)=a_{3}$ is equivalent to

$$
\begin{aligned}
& \frac{\beta_{0}\left(f_{K} \delta_{K} \psi+f_{G} \delta_{G} p_{K}\right)+\frac{\beta_{0} f_{K} \delta_{K}}{\beta_{1} \delta_{K}^{2}} \Psi-2 q \Psi}{\Phi+\frac{\beta_{1} p_{K}+2 f_{K}^{2}}{\beta_{1} \delta_{K}^{2}} \Psi}=\frac{\beta_{0} \delta_{K}(-D)}{(-Q)}-\frac{\beta_{0} p_{K} f_{G}}{(-Q)} \sqrt{\frac{\Psi}{\Phi} \cdot \frac{\beta_{1} \delta_{K}^{2}}{\beta_{1} p_{K}+2 f_{K}^{2}}} \\
\Leftrightarrow \quad & \left(f_{K} \delta_{K} \psi+f_{G} \delta_{G} p_{K}+\frac{f_{K} \delta_{K}}{\beta_{1} \delta_{K}^{2}} \Psi\right)(-Q)+\delta_{K} D\left(\Phi+\frac{\beta_{1} p_{K}+2 f_{K}^{2}}{\beta_{1} \delta_{K}^{2}} \Psi\right) \\
= & -\frac{2 q \Psi Q}{\beta_{0}}-p_{K} f_{G} \sqrt{\frac{\Psi}{\Phi} \cdot \frac{\beta_{1} \delta_{K}^{2}}{\beta_{1} p_{K}+2 f_{K}^{2}}} \cdot\left(\Phi+\frac{\beta_{1} p_{K}+2 f_{K}^{2}}{\beta_{1} \delta_{K}^{2}} \Psi\right),
\end{aligned}
$$

where the left-hand side of the latter equality equals

$$
\begin{aligned}
& \left(f_{K} \delta_{K} \psi+f_{G} \delta_{G} p_{K}\right)\left(-\beta_{1} \delta_{G} p_{K}-2 f_{K} D\right)+\delta_{K} D\left(\beta_{1} p_{K} \psi+2 f_{K}^{2} \psi+2 f_{G}^{2} p_{K}\right) \\
& +\left[\frac{f_{K} \delta_{K}}{\beta_{1} \delta_{K}^{2}} \Psi(-Q)+\delta_{K} D \frac{\beta_{1} p_{K}+2 f_{K}^{2}}{\beta_{1} \delta_{K}^{2}} \Psi\right] \\
= & \left(f_{K} \delta_{K} \psi+f_{G} \delta_{G} p_{K}\right)\left(-\beta_{1} \delta_{G} p_{K}\right)+\left(f_{G} \delta_{G} p_{K}\right)\left(-2 f_{K} D\right)+\delta_{K} D\left(\beta_{1} p_{K} \psi+2 f_{G}^{2} p_{K}\right) \\
& +\frac{\delta_{K} \Psi}{\beta_{1} \delta_{K}^{2}}\left[f_{K}(-Q)+D\left(\beta_{1} p_{K}+2 f_{K}^{2}\right)\right] \\
= & \beta_{1} p_{K}\left(\delta_{K} \psi f_{K} \delta_{G}-\delta_{K} \psi f_{G} \delta_{K}-f_{K} \delta_{K} \psi \delta_{G}-f_{G} \delta_{G} p_{K} \delta_{G}\right)+\delta_{K} D 2 f_{G}^{2} p_{K}-f_{G} \delta_{G} p_{K} 2 f_{K} D \\
& +\frac{\delta_{K} \Psi}{\beta_{1} \delta_{K}^{2}}\left[-f_{K} \beta_{1} \delta_{G} p_{K}-f_{K} 2 f_{K} D+\left(\beta_{1} p_{K}+2 f_{K}^{2}\right) D\right] \\
= & -\beta_{1} p_{K} f_{G}\left(\delta_{K}^{2} \psi+\delta_{G}^{2} p_{K}\right)+2 D f_{G} p_{K}(-D)+\frac{\delta_{K} \Psi}{\beta_{1} \delta_{K}^{2}}\left[-\beta_{1} p_{K} f_{G} \delta_{K}\right] \\
= & -f_{G} p_{K} \Psi-f_{G} p_{K} \Psi ;
\end{aligned}
$$

thus, $a^{*}\left(\gamma_{B}^{*}\right)=a_{3}$ is equivalent to

$$
\begin{aligned}
& \beta_{0}\left(-2 f_{G} p_{K} \Psi\right)+2 q \Psi Q=-\beta_{0} p_{K} f_{G} \sqrt{\frac{\Psi}{\Phi} \cdot \frac{\beta_{1} \delta_{K}^{2}}{\beta_{1} p_{K}+2 f_{K}^{2}}} \cdot\left(\Phi+\frac{\beta_{1} p_{K}+2 f_{K}^{2}}{\beta_{1} \delta_{K}^{2}} \Psi\right) \\
\Leftrightarrow & -\beta_{0} p_{K} f_{G} \sqrt{\frac{\Psi}{\Phi} \cdot \frac{\beta_{1} \delta_{K}^{2}}{\beta_{1} p_{K}+2 f_{K}^{2}}} \cdot\left(\Phi+\frac{\beta_{1} p_{K}+2 f_{K}^{2}}{\beta_{1} \delta_{K}^{2}} \Psi\right)+2 \beta_{0} f_{G} p_{K} \Psi=2 q \Psi Q \\
\Leftrightarrow & q=\frac{1}{2 \Psi \cdot(-Q)} \cdot \beta_{0} p_{K} f_{G}\left[\sqrt{\frac{\Psi}{\Phi} \cdot \frac{\beta_{1} \delta_{K}^{2}}{\beta_{1} p_{K}+2 f_{K}^{2}}} \cdot\left(\Phi+\frac{\beta_{1} p_{K}+2 f_{K}^{2}}{\beta_{1} \delta_{K}^{2}} \Psi\right)-2 \Psi\right]=q_{3},
\end{aligned}
$$

and $q_{3}>0$ if and only if

$$
\sqrt{\frac{\Psi}{\Phi} \cdot \frac{\beta_{1} \delta_{K}^{2}}{\beta_{1} p_{K}+2 f_{K}^{2}}} \cdot\left(\Phi+\frac{\beta_{1} p_{K}+2 f_{K}^{2}}{\beta_{1} \delta_{K}^{2}} \Psi\right)-2 \Psi>0
$$




$$
\begin{aligned}
& \Leftrightarrow \quad \sqrt{\frac{\Psi}{\Phi} \cdot \frac{\beta_{1} \delta_{K}^{2}}{\beta_{1} p_{K}+2 f_{K}^{2}}}>\frac{2 \Psi}{\Phi+\frac{\beta_{1} p_{K}+2 f_{K}^{2}}{\beta_{1} \delta_{K}^{2}} \Psi} \\
& \Leftrightarrow \quad \frac{\Psi}{\Phi} \cdot \frac{\beta_{1} \delta_{K}^{2}}{\beta_{1} p_{K}+2 f_{K}^{2}}>\frac{4 \Psi^{2}}{\left(\Phi+\frac{\beta_{1} p_{K}+2 f_{K}^{2}}{\beta_{1} \delta_{K}^{2}} \Psi\right)^{2}} \\
& \Leftrightarrow \quad \beta_{1} \delta_{K}^{2}\left(\Phi+\frac{\beta_{1} p_{K}+2 f_{K}^{2}}{\beta_{1} \delta_{K}^{2}} \Psi\right)^{2}>4 \Psi \Phi \cdot\left(\beta_{1} p_{K}+2 f_{K}^{2}\right) \\
& \Leftrightarrow \quad\left[\beta_{1} \delta_{K}^{2} \Phi+\left(\beta_{1} p_{K}+2 f_{K}^{2}\right) \Psi\right]^{2}-4 \Psi \Phi \cdot\left(\beta_{1} p_{K}+2 f_{K}^{2}\right) \beta_{1} \delta_{K}^{2}>0 \\
& \Leftrightarrow \quad\left[\beta_{1} \delta_{K}^{2} \Phi-\left(\beta_{1} p_{K}+2 f_{K}^{2}\right) \Psi\right]^{2}>0,
\end{aligned}
$$

a true statement. Next, in order to see that $a^{*}\left(\gamma_{B}^{*}\right)>\frac{2 \beta_{0} \delta_{G}(-D)}{R\left(\gamma_{B}^{*}\right)}$, note that $2 \beta_{0} \delta_{G} D+R\left(\gamma_{B}^{*}\right) a^{*}\left(\gamma_{B}^{*}\right)>0$ is equivalent to

$$
\begin{aligned}
& \beta_{0} \delta_{G} D+\left(\beta_{1} \delta_{K} \psi+\frac{\Psi}{\delta_{K}}-2 f_{G} D\right)\left(\frac{\beta_{0}\left(f_{K} \delta_{K} \psi+f_{G} \delta_{G} p_{K}\right)+\frac{\beta_{0} f_{K}}{\beta_{1} \delta_{K}} \Psi-2 q \Psi}{\Phi+\frac{\left(\beta_{1} p_{K}+2 f_{K}^{2}\right)}{\beta_{1} \delta_{K}^{2}} \Psi}\right)>0 \\
\Leftrightarrow & \delta_{G} D\left(\Phi+\frac{\left(\beta_{1} p_{K}+2 f_{K}^{2}\right)}{\beta_{1} \delta_{K}^{2}} \Psi\right)+\left(\left(f_{K} \delta_{K} \psi+f_{G} \delta_{G} p_{K}\right)+\frac{f_{K}}{\beta_{1} \delta_{K}} \Psi\right)\left(\left(\beta_{1} \delta_{K} \psi-2 f_{G} D\right)+\frac{\Psi}{\delta_{K}}\right) \\
& >\frac{q}{\beta_{0}} 2 \Psi \cdot\left(\left[\beta_{1} \delta_{K} \psi-2 f_{G}\left(\delta_{G} f_{K}-\delta_{K} f_{G}\right)\right]+\frac{\Psi}{\delta_{K}}\right) .
\end{aligned}
$$

Since $\delta_{G}\left(\delta_{G} f_{K}-\delta_{K} f_{G}\right)\left[2 f_{G}^{2} p_{K}+\left(\beta_{1} p_{K}+2 f_{K}^{2}\right) \psi\right]+\left(f_{K} \delta_{K} \psi+f_{G} \delta_{G} p_{K}\right)\left[\beta_{1} \delta_{K} \psi-2 f_{G}\left(\delta_{G} f_{K}-\delta_{K} f_{G}\right)\right]=f_{K} \psi \Psi$, the left hand side of the latter inequality equals

$$
\begin{aligned}
& f_{K} \psi \Psi+\delta_{G} D \frac{\left(\beta_{1} p_{K}+2 f_{K}^{2}\right)}{\beta_{1} \delta_{K}^{2}} \Psi+\frac{f_{K}}{\beta_{1} \delta_{K}} \Psi \cdot\left(\beta_{1} \delta_{K} \psi-2 f_{G} D\right)+\frac{f_{K}}{\beta_{1} \delta_{K}^{2}} \Psi^{2}+\left(f_{K} \delta_{K} \psi+f_{G} \delta_{G} p_{K}\right) \frac{\Psi}{\delta_{K}} \\
= & \Psi \cdot\left[\delta_{G}\left(\delta_{G} f_{K}-\delta_{K} f_{G}\right) \frac{\left(\beta_{1} p_{K}+2 f_{K}^{2}\right)}{\beta_{1} \delta_{K}^{2}}+f_{K} \psi+\frac{f_{K}}{\beta_{1} \delta_{K}} R\left(\gamma_{B}^{*}\right)+\left(f_{K} \delta_{K} \psi+f_{G} \delta_{G} p_{K}\right) \frac{1}{\delta_{K}}\right] \\
= & \Psi \frac{f_{K}}{\beta_{1} \delta_{K}} R\left(\gamma_{B}^{*}\right)+\Psi \cdot\left[\delta_{G}\left(\delta_{G} f_{K}-\delta_{K} f_{G}\right) \frac{\left(\beta_{1} p_{K}+2 f_{K}^{2}\right)}{\beta_{1} \delta_{K}^{2}}+f_{K} \psi+\left(f_{K} \delta_{K} \psi+f_{G} \delta_{G} p_{K}\right) \frac{1}{\delta_{K}}\right] \\
= & \Psi \frac{f_{K}}{\beta_{1} \delta_{K}} R\left(\gamma_{B}^{*}\right)+\Psi \cdot\left[\frac{f_{K}\left[\beta_{1}\left(\delta_{G}^{2} p_{K}+\delta_{K}^{2} \psi\right)+2 f_{K} \delta_{G}\left(f_{K} \delta_{G}-\delta_{K} f_{G}\right)\right]}{\beta_{1} \delta_{K}^{2}}+\frac{\left.\beta_{1} \delta_{K}^{2} f_{K} \psi\right]}{\beta_{1} \delta_{K}^{2}}\right] \\
= & \Psi \frac{f_{K}}{\beta_{1} \delta_{K}} R\left(\gamma_{B}^{*}\right)+\Psi \frac{f_{K}}{\beta_{1} \delta_{K}}\left[\frac{\beta_{1}\left(\delta_{G}^{2} p_{K}+2 \delta_{K}^{2} \psi\right)+2 f_{K} \delta_{G}\left(f_{K} \delta_{G}-\delta_{K} f_{G}\right)}{\delta_{K}}+\beta_{1} \delta_{K} \psi\right] \\
= & \Psi \frac{f_{K}}{\beta_{1} \delta_{K}} R\left(\gamma_{B}^{*}\right)+\Psi \cdot\left[\frac{f_{K}}{\beta_{1} \delta_{K}} R\left(\gamma_{B}^{*}\right)\right] .
\end{aligned}
$$

Thus, $2 \beta_{0} \delta_{G} D+R\left(\gamma_{B}^{*}\right) a^{*}\left(\gamma_{B}^{*}\right)>0$ is equivalent to $\frac{f_{K}}{\beta_{1} \delta_{K}} R\left(\gamma_{B}^{*}\right)+\frac{f_{K}}{\beta_{1} \delta_{K}} R\left(\gamma_{B}^{*}\right)>2 \frac{q}{\beta_{0}} R\left(\gamma_{B}^{*}\right) \Leftrightarrow q<\frac{\beta_{0} f_{K}}{\beta_{1} \delta_{K}}$, which is satisfied since $q<\bar{q} \stackrel{\text { def }}{=} \frac{\beta_{0} f_{K} \psi}{R} \leq \frac{\beta_{0} f_{K}}{\beta_{1} \delta_{K}}$.

In order to see that $a_{3}>\frac{2 \beta_{0} \delta_{G}(-D)}{R\left(\gamma_{B}^{*}\right)}$, note that $a_{3}>a_{1}>\frac{2 \beta_{0} \delta_{G}(-D)}{R}>\frac{2 \beta_{0} \delta_{G}(-D)}{R+\frac{\Psi}{\delta_{K}}}=\frac{2 \beta_{0} \delta_{G}(-D)}{R\left(\gamma_{B}^{*}\right)}$.

If $q \geq q_{3}$, then the gas supplier's profits are

$$
\begin{aligned}
& \left(\gamma_{B}^{*}-\psi\right) \frac{\left[2 \beta_{0} \delta_{K}\left(\delta_{K} f_{G}-\delta_{G} f_{K}\right)+Q \cdot a_{M}^{*}\right]^{2}}{4 \Psi\left(\gamma_{B}^{*}\right)^{2}} \\
= & \frac{\Psi}{\beta_{1} \delta_{K}^{2} 4(2 \Psi)^{2}}\left[2 \beta_{0} \delta_{K}\left(\delta_{K} f_{G}-\delta_{G} f_{K}\right)+Q \frac{2\left[\beta_{0}\left(f_{K} \delta_{K} \psi+f_{G} \delta_{G} p_{K}\right)+\frac{\beta_{0} f_{K}}{\beta_{1} \delta_{K}} \Psi-2 q \Psi\right]}{\Phi+\left(\beta_{1} p_{K}+2 f_{K}^{2}\right) \frac{\Psi}{\beta_{1} \delta_{K}^{2}}}\right]^{2} \\
= & \frac{1}{16 \beta_{1} \delta_{K}^{2} \Psi \cdot\left[\Phi+\left(\beta_{1} p_{K}+2 f_{K}^{2}\right) \frac{\Psi}{\beta_{1} \delta_{K}^{2}}\right]^{2}}\left[2 \beta_{0} \delta_{K}\left(\delta_{K} f_{G}-\delta_{G} f_{K}\right)\left(\Phi+\left(\beta_{1} p_{K}+2 f_{K}^{2}\right) \frac{\Psi}{\beta_{1} \delta_{K}^{2}}\right)\right. \\
\left.+2 Q\left(\beta_{0}\left(f_{K} \delta_{K} \psi+f_{G} \delta_{G} p_{K}\right)+\frac{\beta_{0} f_{K}}{\beta_{1} \delta_{K}} \Psi\right)-2 Q 2 q \Psi\right]^{2} & \frac{\left(2 \beta_{0} f_{G} p_{K} 2 \Psi-2 Q 2 q \Psi\right)^{2}}{16 \beta_{1} \delta_{K}^{2} \Psi \cdot\left[\Phi+\left(\beta_{1} p_{K}+2 f_{K}^{2}\right) \frac{\Psi}{\beta_{1} \delta_{K}^{2}}\right]^{2}}=\frac{\beta_{1} \delta_{K}^{2} \Psi \cdot\left(\beta_{0} f_{G} p_{K}-q Q\right)^{2}}{\left[\beta_{1} \delta_{K}^{2} \Phi+\left(\beta_{1} p_{K}+2 f_{K}^{2}\right) \Psi\right]^{2}},
\end{aligned}
$$

where the latter equality follows from

$$
\begin{aligned}
& \beta_{1} \delta_{K}^{2}\left[\delta_{K}\left(\delta_{K} f_{G}-\delta_{G} f_{K}\right)\left(\Phi+\left(\beta_{1} p_{K}+2 f_{K}^{2}\right) \frac{\Psi}{\beta_{1} \delta_{K}^{2}}\right)+Q\left(\left(f_{K} \delta_{K} \psi+f_{G} \delta_{G} p_{K}\right)+\frac{f_{K}}{\beta_{1} \delta_{K}} \Psi\right)\right] \\
= & \delta_{K}\left(\delta_{K} f_{G}-\delta_{G} f_{K}\right) \beta_{1} \delta_{K}^{2}\left(\beta_{1} p_{K}+2 f_{K}^{2}\right) \psi+\delta_{K}\left(\delta_{K} f_{G}-\delta_{G} f_{K}\right) \beta_{1} \delta_{K}^{2} 2 f_{G}^{2} p_{K}
\end{aligned}
$$




$$
\begin{aligned}
& +\delta_{K}\left(\delta_{K} f_{G}-\delta_{G} f_{K}\right)\left(\beta_{1} p_{K}+2 f_{K}^{2}\right) \beta_{1} \delta_{G}^{2} p_{K}+\delta_{K}\left(\delta_{K} f_{G}-\delta_{G} f_{K}\right)\left(\beta_{1} p_{K}+2 f_{K}^{2}\right) \beta_{1} \delta_{K}^{2} \psi \\
& +\delta_{K}\left(\delta_{K} f_{G}-\delta_{G} f_{K}\right)\left(\beta_{1} p_{K}+2 f_{K}^{2}\right) 2 D^{2}+\left[\beta_{1} \delta_{G} p_{K}+2 f_{K}\left(f_{K} \delta_{G}-f_{G} \delta_{K}\right)\right] f_{K} \delta_{K} \psi \beta_{1} \delta_{K}^{2} \\
& +\left[\beta_{1} \delta_{G} p_{K}+2 f_{K}\left(f_{K} \delta_{G}-f_{G} \delta_{K}\right)\right] f_{G} \delta_{G} p_{K} \beta_{1} \delta_{K}^{2}+\left[\beta_{1} \delta_{G} p_{K}+2 f_{K}\left(f_{K} \delta_{G}-f_{G} \delta_{K}\right)\right] f_{K} \delta_{K} \beta_{1} \delta_{G}^{2} p_{K} \\
& +\left[\beta_{1} \delta_{G} p_{K}+2 f_{K}\left(f_{K} \delta_{G}-f_{G} \delta_{K}\right)\right] f_{K} \delta_{K} \beta_{1} \delta_{K}^{2} \psi+\left[\beta_{1} \delta_{G} p_{K}+2 f_{K}\left(f_{K} \delta_{G}-f_{G} \delta_{K}\right)\right] f_{K} \delta_{K} 2 D^{2} \\
= & \delta_{K}\left(\delta_{K} f_{G}\right) \beta_{1} \delta_{K}^{2}\left(\beta_{1} p_{K}\right) \psi+\delta_{K}\left(\delta_{K} f_{G}-\delta_{G} f_{K}\right) \beta_{1} \delta_{K}^{2} 2 f_{G}^{2} p_{K}+\delta_{K}\left(\delta_{K} f_{G}\right)\left(\beta_{1} p_{K}\right) \beta_{1} \delta_{G}^{2} p_{K} \\
& +\delta_{K}\left(\delta_{K} f_{G}\right)\left(\beta_{1} p_{K}\right) \beta_{1} \delta_{K}^{2} \psi+\delta_{K}\left(\delta_{K} f_{G}\right)\left(\beta_{1} p_{K}\right) 2 D^{2}+\left[\beta_{1} \delta_{G} p_{K}+2 f_{K}\left(f_{K} \delta_{G}-f_{G} \delta_{K}\right)\right] f_{G} \delta_{G} p_{K} \beta_{1} \delta_{K}^{2} \\
= & \beta_{1} \delta_{K}^{2} f_{G} p_{K}\left[2 f_{G} \delta_{K}\left(\delta_{K} f_{G}-\delta_{G} f_{K}\right)+2 \beta_{1} \delta_{K}^{2} \psi+2 \beta_{1} \delta_{G}^{2} p_{K}+2 f_{K} \delta_{G}\left(f_{K} \delta_{G}-f_{G} \delta_{K}\right)+2 D^{2}\right] \\
= & \beta_{1} \delta_{K}^{2} f_{G} p_{K} 2 \Psi .
\end{aligned}
$$

If $q<q_{3}$, then the gas supplier's profits are

$$
\left(\gamma_{B}^{*}-\psi\right) \frac{\left[2 \beta_{0} \delta_{K}(-D)+Q a_{3}\right]^{2}}{4 \Psi\left(\gamma_{B}^{*}\right)^{2}}=\left(\gamma_{B}^{*}-\psi\right) \frac{\left[2 \beta_{0} \delta_{K}(-D)+Q a_{M}^{*}\left(q_{3}\right)\right]^{2}}{4 \Psi\left(\gamma_{B}^{*}\right)^{2}}=\frac{\beta_{1} \delta_{K}^{2} \Psi \cdot\left(\beta_{0} f_{G} p_{K}-q_{3} Q\right)^{2}}{\left[\beta_{1} \delta_{K}^{2} \Phi+\left(\beta_{1} p_{K}+2 f_{K}^{2}\right) \Psi\right]^{2}},
$$

which equals $\frac{\left(\beta_{0} f_{G} p_{K}\right)^{2}}{4 \Phi \cdot\left(\beta_{1} p_{K}+2 f_{K}^{2}\right)}$ since

$$
\begin{aligned}
\beta_{0} f_{G} p_{K}+q_{3}(-Q) & =\beta_{0} f_{G} p_{K}+(-Q) \frac{\beta_{0} f_{G} p_{K}}{2 \Psi \cdot(-Q)}\left[\sqrt{\frac{\Psi}{\Phi} \cdot \frac{\beta_{1} \delta_{K}^{2}}{\beta_{1} p_{K}+2 f_{K}^{2}}} \cdot\left(\Phi+\frac{\beta_{1} p_{K}+2 f_{K}^{2}}{\beta_{1} \delta_{K}^{2}} \Psi\right)-2 \Psi\right] \\
& =\frac{\beta_{0} f_{G} p_{K}}{2 \Psi}\left[2 \Psi+\sqrt{\frac{\Psi}{\Phi} \cdot \frac{\beta_{1} \delta_{K}^{2}}{\beta_{1} p_{K}+2 f_{K}^{2}}} \cdot\left(\Phi+\frac{\beta_{1} p_{K}+2 f_{K}^{2}}{\beta_{1} \delta_{K}^{2}} \Psi\right)-2 \Psi\right] .
\end{aligned}
$$

\section{C.5 Calculations for Lemma 2.1g}

Note that $W_{A}^{C}(q)<W_{B}^{C}(q)$ is equivalent to (the true statement)

$$
\begin{array}{ll} 
& \frac{-2 \beta_{0} \beta_{1} q\left(f_{K} \delta_{K} \psi+f_{G} \delta_{G} p_{K}\right)+\beta_{0}^{2}\left(f_{K}^{2} \psi+f_{G}^{2} p_{K}\right)}{\beta_{1} \Phi}<\frac{\beta_{1}\left[\beta_{0}\left(f_{K} \delta_{K} \psi+f_{G} \delta_{G} p_{K}\right)-q \Psi\right]^{2}+\beta_{0}^{2} D^{2} \Phi}{\beta_{1} \Psi \Phi} \\
\Leftrightarrow & \beta_{0}^{2}\left(f_{K}^{2} \psi+f_{G}^{2} p_{K}\right) \Psi<\beta_{1} \beta_{0}^{2}\left(f_{K} \delta_{K} \psi+f_{G} \delta_{G} p_{K}\right)^{2}+\beta_{1} q^{2} \Psi^{2}+\beta_{0}^{2} D^{2} \Phi \\
\Leftrightarrow & \beta_{1} q^{2} \Psi^{2}+\beta_{1} \beta_{0}^{2}\left(f_{K} \delta_{K} \psi+f_{G} \delta_{G} p_{K}\right)^{2}+\beta_{0}^{2} D^{2} \beta_{1} p_{K} \psi-\beta_{0}^{2}\left(f_{K}^{2} \psi+f_{G}^{2} p_{K}\right) \beta_{1}\left(\delta_{G}^{2} p_{K}+\delta_{K}^{2} \psi\right)>0 \\
\Leftrightarrow & \beta_{1} q^{2} \Psi^{2}+\beta_{1} \beta_{0}^{2} \cdot\left[\left(f_{K} \delta_{K} \psi+f_{G} \delta_{G} p_{K}\right)^{2}+\psi p_{K} D^{2}-\left(f_{K}^{2} \psi+f_{G}^{2} p_{K}\right)\left(\delta_{G}^{2} p_{K}+\delta_{K}^{2} \psi\right)\right]>0 \\
\Leftrightarrow & \beta_{1} q^{2} \Psi^{2}>0 .
\end{array}
$$

\section{C.6 Calculations for Lemma $2.1 \mathrm{~h}$}

First, note that $W_{B}^{M}(q)-W_{A}^{M}(q)=W_{B}^{M}(q)-W_{p=0}^{M}(q)=W_{B}^{M}(q)-\left[W^{M}(q)-\frac{1}{2 \Phi}\left(\Psi+\frac{\beta_{1} \delta_{K}^{2}}{\beta_{1} p_{K}+2 f_{K}^{2}} \Phi\right) q^{2}\right]$

$$
=\left[W_{B}^{M}(q)-W^{M}(q)\right]+\frac{1}{2 \Phi}\left(\Psi+\frac{\beta_{1} \delta_{K}^{2}}{\beta_{1} p_{K}+2 f_{K}^{2}} \Phi\right) q^{2}=\frac{C_{0}+C_{1} \cdot q+\beta_{1} \Psi 4 \Psi \Phi \cdot q^{2}}{2 \beta_{1} \Psi \Phi \cdot\left(\Phi+\frac{\beta_{1} p_{K}+2 f_{K}^{2}}{\beta_{1} \delta_{K}^{2}} \Psi\right)},
$$

where $C_{1}$ and $C_{0}$ are as defined in the calculations for Lemma $3 \mathrm{a}$. Therefore,

$$
W_{B}^{M}(q)-W_{A}^{M}(q)=\frac{-\beta_{0}^{2} p_{K}^{2} f_{G}^{2} Q^{2}+2 \beta_{0} p_{K} f_{G} Q^{3} \cdot q+4 \Psi \Phi \cdot\left(\beta_{1} p_{K}+2 f_{K}^{2}\right) \beta_{1} \delta_{K}^{2} \cdot q^{2}}{2 \Phi \cdot\left(\Phi+\frac{\beta_{1} p_{K}+2 f_{K}^{2}}{\beta_{1} \delta_{K}^{2}} \Psi\right)\left(\beta_{1} p_{K}+2 f_{K}^{2}\right) \beta_{1} \delta_{K}^{2}} .
$$

The positive argument at which this convex quadratic function of $q$ takes the value zero is given by

$$
\begin{aligned}
& \frac{\beta_{0} p_{K} f_{G}\left(-Q^{3}-Q \sqrt{Q^{4}+4 \Psi \Phi \cdot\left(\beta_{1} p_{K}+2 f_{K}^{2}\right) \beta_{1} \delta_{K}^{2}}\right)}{4 \Psi \Phi \cdot\left(\beta_{1} p_{K}+2 f_{K}^{2}\right) \beta_{1} \delta_{K}^{2}} \\
= & \frac{\beta_{0} p_{K} f_{G}\left[-Q^{3}-Q \cdot\left[\beta_{1} \delta_{K}^{2} \Phi+\left(\beta_{1} p_{K}+2 f_{K}^{2}\right) \Psi\right]\right]}{4 \Psi \Phi \cdot\left(\beta_{1} p_{K}+2 f_{K}^{2}\right) \beta_{1} \delta_{K}^{2}} \\
= & \frac{\beta_{0} p_{K} f_{G}(-Q)\left[\left[\left(\beta_{1} p_{K}+2 f_{K}^{2}\right) \Psi-\beta_{1} \delta_{K}^{2} \Phi\right]+\left[\beta_{1} \delta_{K}^{2} \Phi+\left(\beta_{1} p_{K}+2 f_{K}^{2}\right) \Psi\right]\right]}{4 \Psi \Phi \cdot\left(\beta_{1} p_{K}+2 f_{K}^{2}\right) \beta_{1} \delta_{K}^{2}} \\
= & \frac{\beta_{0} p_{K} f_{G}(-Q)\left[2\left(\beta_{1} p_{K}+2 f_{K}^{2}\right) \Psi\right]}{4 \Psi \Phi \cdot\left(\beta_{1} p_{K}+2 f_{K}^{2}\right) \beta_{1} \delta_{K}^{2}}=\tilde{q}(Q) .
\end{aligned}
$$

Finally, $q_{3}<\tilde{q}(Q)$ is equivalent to (the true statement)

$$
\begin{aligned}
& \frac{1}{(-Q)}\left[\sqrt{\frac{\Psi}{\Phi} \frac{\beta_{1} \delta_{K}^{2}}{\left(\beta_{1} p_{K}+2 f_{K}^{2}\right)}}\left(\Phi+\frac{\beta_{1} p_{K}+2 f_{K}^{2}}{\beta_{1} \delta_{K}^{2}} \Psi\right)-2 \Psi\right]<\frac{-Q^{3}-Q\left[\beta_{1} \delta_{K}^{2} \Phi+\left(\beta_{1} p_{K}+2 f_{K}^{2}\right) \Psi\right]}{2 \Phi \cdot\left(\beta_{1} p_{K}+2 f_{K}^{2}\right) \beta_{1} \delta_{K}^{2}} \\
\Leftrightarrow \quad & {\left[\sqrt{\frac{\Psi}{\Phi} \frac{\beta_{1} \delta_{K}^{2}}{\left(\beta_{1} p_{K}+2 f_{K}^{2}\right)}}\left(\Phi+\frac{\beta_{1} p_{K}+2 f_{K}^{2}}{\beta_{1} \delta_{K}^{2}} \Psi\right)-2 \Psi\right] 2 \Phi \cdot\left(\beta_{1} p_{K}+2 f_{K}^{2}\right) \beta_{1} \delta_{K}^{2} } \\
& <Q^{4}+Q^{2}\left[\beta_{1} \delta_{K}^{2} \Phi+\left(\beta_{1} p_{K}+2 f_{K}^{2}\right) \Psi\right]
\end{aligned}
$$




$$
\begin{array}{lll}
\Leftrightarrow & \sqrt{4 \Psi \Phi \cdot\left(\beta_{1} p_{K}+2 f_{K}^{2}\right) \beta_{1} \delta_{K}^{2}} \cdot\left[\beta_{1} \delta_{K}^{2} \Phi+\left(\beta_{1} p_{K}+2 f_{K}^{2}\right) \Psi\right]-4 \Psi \Phi \cdot\left(\beta_{1} p_{K}+2 f_{K}^{2}\right) \beta_{1} \delta_{K}^{2} \\
& -Q^{4}-Q^{2}\left[\beta_{1} \delta_{K}^{2} \Phi+\left(\beta_{1} p_{K}+2 f_{K}^{2}\right) \Psi\right]<0 \\
\Leftrightarrow & \sqrt{4 \Psi \Phi \cdot\left(\beta_{1} p_{K}+2 f_{K}^{2}\right) \beta_{1} \delta_{K}^{2}} \cdot\left[\beta_{1} \delta_{K}^{2} \Phi+\left(\beta_{1} p_{K}+2 f_{K}^{2}\right) \Psi\right]-\left[\beta_{1} \delta_{K}^{2} \Phi+\left(\beta_{1} p_{K}+2 f_{K}^{2}\right) \Psi\right]^{2} \\
& -Q^{2}\left[\beta_{1} \delta_{K}^{2} \Phi+\left(\beta_{1} p_{K}+2 f_{K}^{2}\right) \Psi\right]<0 \\
\Leftrightarrow & -\left[\beta_{1} \delta_{K}^{2} \Phi+\left(\beta_{1} p_{K}+2 f_{K}^{2}\right) \Psi\right] \cdot\left[\left[\beta_{1} \delta_{K}^{2} \Phi+\left(\beta_{1} p_{K}+2 f_{K}^{2}\right) \Psi\right]-\sqrt{4 \Psi \Phi \cdot\left(\beta_{1} p_{K}+2 f_{K}^{2}\right) \beta_{1} \delta_{K}^{2}}+Q^{2}\right]<0 \\
\Leftrightarrow & {\left[\beta_{1} \delta_{K}^{2} \Phi+\left(\beta_{1} p_{K}+2 f_{K}^{2}\right) \Psi\right]-\sqrt{4 \Psi \Phi \cdot\left(\beta_{1} p_{K}+2 f_{K}^{2}\right) \beta_{1} \delta_{K}^{2}}+Q^{2}>0} \\
\Leftrightarrow & {\left[\beta_{1} \delta_{K}^{2} \Phi+\left(\beta_{1} p_{K}+2 f_{K}^{2}\right) \Psi\right]-\sqrt{4 \Psi \Phi \cdot\left(\beta_{1} p_{K}+2 f_{K}^{2}\right) \beta_{1} \delta_{K}^{2}}-\left[\beta_{1} \delta_{K}^{2} \Phi-\left(\beta_{1} p_{K}+2 f_{K}^{2}\right) \Psi\right]>0} \\
\Leftrightarrow & {\left[\beta_{1} \delta_{K}^{2} \Phi+\left(\beta_{1} p_{K}+2 f_{K}^{2}\right) \Psi\right]-\left[\beta_{1} \delta_{K}^{2} \Phi-\left(\beta_{1} p_{K}+2 f_{K}^{2}\right) \Psi\right]>\sqrt{4 \Psi \Phi \cdot\left(\beta_{1} p_{K}+2 f_{K}^{2}\right) \beta_{1} \delta_{K}^{2}}} \\
\Leftrightarrow & 2\left(\beta_{1} p_{K}+2 f_{K}^{2}\right) \Psi>\sqrt{4 \Psi \Phi \cdot\left(\beta_{1} p_{K}+2 f_{K}^{2}\right) \beta_{1} \delta_{K}^{2}} \Leftrightarrow \quad \Leftrightarrow\left(\beta_{1} p_{K}+2 f_{K}^{2}\right)^{2} \Psi^{2}>4 \Psi \Phi \cdot\left(\beta_{1} p_{K}+2 f_{K}^{2}\right) \beta_{1} \delta_{K}^{2} \\
\Leftrightarrow & \left(\beta_{1} p_{K}+2 f_{K}^{2}\right) \Psi>\beta_{1} \delta_{K}^{2} \Phi \Leftrightarrow \quad \Leftrightarrow \quad \beta_{1} \delta_{K}^{2} \Phi-\left(\beta_{1} p_{K}+2 f_{K}^{2}\right) \Psi<0 \\
\Leftrightarrow & -Q^{2}<0 .
\end{array}
$$

\section{C.7 Calculations for Lemma 2.2c}

To see that $a_{5}<a_{4}<a_{2}<a_{1}<a_{0}$, follow the corresponding calculations for Lemma 2.1c. To see that $a_{-1}<a_{4}$, note that in our calculations for Lemma $2.1 \mathrm{f}$ we show that

$$
a_{4}-\frac{4 \Psi}{\Phi+\frac{\beta_{1} p_{K}+2 f_{K}^{2}}{\beta_{1} \delta_{K}^{2}} \Psi} q\left(=a^{*}\left(\gamma_{B}^{*}\right)\right)>\frac{2 \beta_{0} \delta_{G}(-D)}{R\left(\gamma_{B}^{*}\right)}\left(=a_{-1}\right) \Leftrightarrow q<\frac{\beta_{0} f_{K}}{\beta_{1} \delta_{K}} .
$$

To see that $\hat{\gamma}(a)$ is decreasing in $a$, cf. our calculations for Lemma 2.1c.

Finally, to see that $\frac{(\hat{\gamma}(a)-\psi) \cdot\left[2 \beta_{0} \delta_{K}(-D)+a Q\right]^{2}}{4 \Psi(\hat{\gamma}(a))^{2}}=\frac{(\hat{\gamma}(a)-\psi) \cdot\left(\beta_{0} p_{K} f_{G}\right)^{2}}{\Phi(\hat{\gamma}(a))^{2}}=\hat{\Gamma}_{A}(a)$,

where $\hat{\Gamma}_{A}(a) \stackrel{\text { def }}{=} \frac{\left[\Phi \cdot a-2 \beta_{0}\left(f_{K} \delta_{K} \psi+f_{G} \delta_{G} p_{K}\right)\right] \cdot\left[2 \beta_{0} f_{K} \delta_{K}-\left(\beta_{1} p_{K}+2 f_{K}^{2}\right) \cdot a\right]}{4 Q^{2}}$, note that the first of the two equalities is satisfied if $\hat{\gamma}(a)=\psi$, which is equivalent to $a=a_{1}$. If $a \neq a_{1}$, then this equality is equivalent to

$$
\begin{array}{ll} 
& \frac{\left[2 \beta_{0} \delta_{K}(-D)+a Q\right]^{2}}{4 \Psi(\hat{\gamma}(a))^{2}}=\frac{\left(\beta_{0} p_{K} f_{G}\right)^{2}}{\Phi(\hat{\gamma}(a))^{2}} \\
\Leftrightarrow \quad & \Phi(\hat{\gamma}(a))^{2}\left[2 \beta_{0} \delta_{K}(-D)+a Q\right]^{2}=\left(\beta_{0} p_{K} f_{G}\right)^{2} 4 \Psi(\hat{\gamma}(a))^{2} \\
\Leftrightarrow \quad & \Phi(\hat{\gamma}(a))\left[2 \beta_{0} \delta_{K}(-D)+a Q\right]=\left(\beta_{0} p_{K} f_{G}\right) 2 \Psi(\hat{\gamma}(a)) \\
\Leftrightarrow \quad & \left(\beta_{1} p_{K}+2 f_{K}^{2}\right)\left[2 \beta_{0} \delta_{K}(-D)+a Q\right] \cdot \hat{\gamma}(a)-\beta_{0} p_{K} f_{G} 2 \beta_{1} \delta_{K}^{2} \cdot \hat{\gamma}(a) \\
& \quad-2 f_{G}^{2} p_{K}\left[2 \beta_{0} \delta_{K}(-D)+a Q\right]+\beta_{0} p_{K} f_{G} 2\left(\beta_{1} \delta_{G}^{2} p_{K}+2 D^{2}\right) \\
\Leftrightarrow \quad & {\left[\left(\beta_{1} p_{K}+2 f_{K}^{2}\right) a-2 \beta_{0} \delta_{K} f_{K}\right] Q \cdot \hat{\gamma}(a)=Q\left[-2 f_{G}^{2} p_{K} a+2 \beta_{0} p_{K} f_{G} \delta_{G}\right]} \\
\Leftrightarrow \quad & \hat{\gamma}(a)=\frac{2 f_{G}^{2} p_{K} \cdot a-2 \beta_{0} f_{G} \delta_{G} p_{K}}{2 \beta_{0} \delta_{K} f_{K}-a \cdot\left(\beta_{1} p_{K}+2 f_{K}^{2}\right)},
\end{array}
$$

a true statement. The second of the two equalities follows from

$$
\begin{aligned}
& \frac{(\hat{\gamma}(a)-\psi) \cdot\left(\beta_{0} p_{K} f_{G}\right)^{2}}{\Phi(\hat{\gamma}(a))^{2}}=\frac{\left(\frac{2 f_{G}^{2} p_{K} \cdot a-2 \beta_{0} f_{G} \delta_{G} p_{K}}{2 \beta_{0} \delta_{K} f_{K}-a \cdot\left(\beta_{1} p_{K}+2 f_{K}^{2}\right)}-\psi\right) \cdot\left(\beta_{0} p_{K} f_{G}\right)^{2}}{\left[\left(\beta_{1} p_{K}+2 f_{K}^{2}\right) \frac{2 f_{G}^{2} p_{K} \cdot a-2 \beta_{0} f_{G} \delta_{G} p_{K}}{2 \beta_{0} \delta_{K} f_{K}-a \cdot\left(\beta_{1} p_{K}+2 f_{K}^{2}\right)}+2 f_{G}^{2} p_{K}\right]^{2}} \\
= & \frac{\left[a \cdot 2 f_{G}^{2} p_{K}-2 \beta_{0} f_{G} \delta_{G} p_{K}-2 \beta_{0} \delta_{K} f_{K} \psi+a \cdot\left(\beta_{1} p_{K}+2 f_{K}^{2}\right) \psi\right] \cdot\left(\beta_{0} p_{K} f_{G}\right)^{2} \cdot\left[2 \beta_{0} \delta_{K} f_{K}-a \cdot\left(\beta_{1} p_{K}+2 f_{K}^{2}\right)\right]}{\left[\left(\beta_{1} p_{K}+2 f_{K}^{2}\right)\left[a \cdot 2 f_{G}^{2} p_{K}-2 \beta_{0} f_{G} \delta_{G} p_{K}\right]+2 f_{G}^{2} p_{K}\left[2 \beta_{0} \delta_{K} f_{K}-a \cdot\left(\beta_{1} p_{K}+2 f_{K}^{2}\right)\right]\right]^{2}} \\
= & \frac{\left[\Phi \cdot a-2 \beta_{0}\left(f_{K} \delta_{K} \psi+f_{G} \delta_{G} p_{K}\right)\right] \cdot\left(\beta_{0} p_{K} f_{G}\right)^{2} \cdot\left[2 \beta_{0} \delta_{K} f_{K}-a \cdot\left(\beta_{1} p_{K}+2 f_{K}^{2}\right)\right]}{\left(-2 \beta_{0} f_{G} p_{K} Q\right)^{2}}=\hat{\Gamma}_{A}(a) .
\end{aligned}
$$

\section{C.8 Calculations for Lemma 2.2e}

Note that $\hat{W}^{M}(a)$ equals

$$
\begin{aligned}
& \frac{2 \beta_{0}\left[\beta_{0}\left(f_{K}^{2} \hat{\gamma}(a)+f_{G}^{2} p_{K}\right)-2 \beta_{1} q\left(f_{K} \delta_{K} \hat{\gamma}(a)+f_{G} \delta_{G} p_{K}\right)\right]}{2 \beta_{1} \Phi(\hat{\gamma}(a))} \\
= & \frac{\beta_{0}}{\beta_{1}} \cdot \frac{\left(\beta_{0} f_{K}^{2}-2 \beta_{1} f_{K} \delta_{K} q\right) \cdot \hat{\gamma}(a)+\beta_{0} f_{G}^{2} p_{K}-2 \beta_{1} q f_{G} \delta_{G} p_{K}}{\left(\beta_{1} p_{K}+2 f_{K}^{2}\right) \cdot \hat{\gamma}(a)+2 f_{G}^{2} p_{K}}
\end{aligned}
$$




$$
\begin{aligned}
= & \frac{\beta_{0}}{\beta_{1}} \cdot \frac{\left[\beta_{0} f_{K}^{2}-2 \beta_{1} f_{K} \delta_{K} q\right]\left[a 2 f_{G}^{2} p_{K}-2 \beta_{0} f_{G} \delta_{G} p_{K}\right]+\left[\beta_{0} f_{G}^{2} p_{K}-2 \beta_{1} q f_{G} \delta_{G} p_{K}\right]\left[2 \beta_{0} f_{K} \delta_{K}-a\left(\beta_{1} p_{K}+2 f_{K}^{2}\right)\right]}{\left(\beta_{1} p_{K}+2 f_{K}^{2}\right)\left[a \cdot 2 f_{G}^{2} p_{K}-2 \beta_{0} f_{G} \delta_{G} p_{K}\right]+2 f_{G}^{2} p_{K}\left[2 \beta_{0} f_{K} \delta_{K}-a \cdot\left(\beta_{1} p_{K}+2 f_{K}^{2}\right)\right]} \\
= & \frac{\beta_{0}}{\beta_{1}} \cdot \frac{f_{G} p_{K}\left[\left(\beta_{0} f_{K}-2 \beta_{1} \delta_{K} q\right) 2 f_{G} f_{K}-\left(\beta_{1} p_{K}+2 f_{K}^{2}\right)\left[\beta_{0} f_{G}-2 \beta_{1} \delta_{G} q\right]\right] a}{\left[\left(\beta_{1} p_{K}+2 f_{K}^{2}\right) 2 f_{G}^{2} p_{K}-\left(\beta_{1} p_{K}+2 f_{K}^{2}\right) 2 f_{G}^{2} p_{K}\right] a-\left(\beta_{1} p_{K}+2 f_{K}^{2}\right) 2 \beta_{0} f_{G} \delta_{G} p_{K}+2 f_{G}^{2} p_{K} 2 \beta_{0} f_{K} \delta_{K}} \\
& +\frac{\beta_{0}}{\beta_{1}} \cdot \frac{-2 \beta_{0} f_{G} \delta_{G} p_{K}\left[\beta_{0} f_{K}^{2}-2 \beta_{1} f_{K} \delta_{K} q\right]+2 \beta_{0} f_{K} \delta_{K}\left[\beta_{0} f_{G}^{2} p_{K}-2 \beta_{1} q f_{G} \delta_{G} p_{K}\right]}{\left[\left(\beta_{1} p_{K}+2 f_{K}^{2}\right) 2 f_{G}^{2} p_{K}-\left(\beta_{1} p_{K}+2 f_{K}^{2}\right) 2 f_{G}^{2} p_{K}\right] a-\left(\beta_{1} p_{K}+2 f_{K}^{2}\right) 2 \beta_{0} f_{G} \delta_{G} p_{K}+2 f_{G}^{2} p_{K} 2 \beta_{0} f_{K} \delta_{K}} \\
= & \frac{\beta_{0}}{\beta_{1}} \cdot \frac{\beta_{1} f_{G} p_{K}\left[2 Q \cdot q-\beta_{0} f_{G} p_{K}\right] a-2 \beta_{0}^{2} p_{K} f_{K} f_{G} D}{-2 \beta_{0} f_{G} p_{K} Q} .
\end{aligned}
$$

\section{C.9 Calculations for Lemma 2.2h}

First, note that

$$
W_{B}^{M}(q)-W_{A}^{M}(q)=\frac{-\beta_{0}^{2} p_{K}^{2} f_{G}^{2} Q^{2}+2 \beta_{0} p_{K} f_{G} Q^{3} \cdot q+4 \Psi \Phi \cdot\left(\beta_{1} p_{K}+2 f_{K}^{2}\right) \beta_{1} \delta_{K}^{2} \cdot q^{2}}{2 \Phi \cdot\left(\Phi+\frac{\beta_{1} p_{K}+2 f_{K}^{2}}{\beta_{1} \delta_{K}^{2}} \Psi\right)\left(\beta_{1} p_{K}+2 f_{K}^{2}\right) \beta_{1} \delta_{K}^{2}}
$$

(cf. our calculations for Lemma 2.1h). The positive argument at which this convex quadratic function of $q$ takes the value zero is given by

$$
\begin{aligned}
& \frac{\beta_{0} p_{K} f_{G}\left[-Q^{3}+Q \sqrt{Q^{4}+4 \Psi \Phi \cdot\left(\beta_{1} p_{K}+2 f_{K}^{2}\right) \beta_{1} \delta_{K}^{2}}\right]}{4 \Psi \Phi \cdot\left(\beta_{1} p_{K}+2 f_{K}^{2}\right) \beta_{1} \delta_{K}^{2}} \\
= & \frac{\beta_{0} p_{K} f_{G}\left[-Q^{3}+Q\left[\beta_{1} \delta_{K}^{2} \Phi+\left(\beta_{1} p_{K}+2 f_{K}^{2}\right) \Psi\right]\right]}{4 \Psi \Phi \cdot\left(\beta_{1} p_{K}+2 f_{K}^{2}\right) \beta_{1} \delta_{K}^{2}} \\
= & \frac{\beta_{0} p_{K} f_{G} Q\left[\left[\beta_{1} \delta_{K}^{2} \Phi-\left(\beta_{1} p_{K}+2 f_{K}^{2}\right) \Psi\right]+\left[\beta_{1} \delta_{K}^{2} \Phi+\left(\beta_{1} p_{K}+2 f_{K}^{2}\right) \Psi\right]\right]}{4 \Psi \Phi \cdot\left(\beta_{1} p_{K}+2 f_{K}^{2}\right) \beta_{1} \delta_{K}^{2}} \\
= & \frac{\beta_{0} p_{K} f_{G} Q\left(2 \beta_{1} \delta_{K}^{2} \Phi\right)}{4 \Psi \Phi \cdot\left(\beta_{1} p_{K}+2 f_{K}^{2}\right) \beta_{1} \delta_{K}^{2}}=\tilde{q}(Q) .
\end{aligned}
$$

Finally, note that $\tilde{q}(Q)<\frac{\beta_{0} f_{G} p_{K}}{2 Q}$ is equivalent to $2 Q^{2}<2\left(\beta_{1} p_{K}+2 f_{K}^{2}\right) \Psi \Leftrightarrow\left(\beta_{1} p_{K}+2 f_{K}^{2}\right) \Psi-\beta_{1} \delta_{K}^{2} \Phi<$ $\left(\beta_{1} p_{K}+2 f_{K}^{2}\right) \Psi$, a true statement.

\section{C.10 Calculations for Lemma 2.3c}

Note that $\bar{C}_{B}=\bar{C}_{A}$ is equivalent to $\frac{\beta_{0}^{2} D^{2}}{4 \beta_{1} \Psi}=\frac{\beta_{0}^{2} p_{K}^{2} f_{G}^{2}}{4\left(\beta_{1} p_{K}+2 f_{K}^{2}\right) \Phi}$, and thus to (the true statement)

$$
\begin{aligned}
& \left(\beta_{1} p_{K}+2 f_{K}^{2}\right) \Phi D^{2}=\Psi \beta_{1} p_{K}^{2} f_{G}^{2} \\
\Leftrightarrow & \Phi(-D)\left[(-D)+f_{K} \delta_{G}\right]=p_{K} f_{G}^{2} \Psi \\
\Leftrightarrow & \Phi(-D) \delta_{K}=p_{K} f_{G} \Psi \quad\left(=p_{K} f_{G}\left[\beta_{1} \delta_{G}^{2} p_{K}+\beta_{1} \delta_{K}^{2} \psi+2 D^{2}\right]\right) \\
\Leftrightarrow & \Phi(-D) \delta_{K}=p_{K} f_{G}\left[\delta_{G} 2 f_{K}(-D)+\beta_{1} \delta_{K}^{2} \psi+2 D^{2}\right] \quad\left(=p_{K} f_{G}\left[\beta_{1} \delta_{K}^{2} \psi+2(-D) f_{G} \delta_{K}\right]\right) \\
\Leftrightarrow & \Phi(-D)=p_{K} f_{G}\left[\beta_{1} \delta_{K} \psi+2(-D) f_{G}\right] \\
\Leftrightarrow & \left(\beta_{1} p_{K} \psi+2 f_{K}^{2} \psi+2 f_{G}^{2} p_{K}\right)(-D)=p_{K} f_{G} \beta_{1} \delta_{K} \psi+2(-D) f_{G}^{2} p_{K} \\
\Leftrightarrow & \left(\beta_{1} p_{K} \psi+2 f_{K}^{2} \psi\right)(-D)=p_{K} f_{G} \beta_{1} \delta_{K} \psi \\
\Leftrightarrow & \beta_{1} p_{K} \psi\left(-f_{K} \delta_{G}\right)+2 f_{K}^{2} \psi(-D)=0 \\
\Leftrightarrow & Q=0 .
\end{aligned}
$$

\section{C.11 Calculations for Lemma 3a}

$$
\begin{aligned}
& {\left[2 \beta_{1} \Psi \Phi \cdot\left(\Phi+\left(\beta_{1} p_{K}+2 f_{K}^{2}\right) \frac{\Psi}{\beta_{1} \delta_{K}^{2}}\right)\right] \cdot\left[W_{B}^{M}-W^{M}\right] } \\
=\quad & \beta_{1}\left[\left(\beta_{0}\left(f_{K} \delta_{K} \psi+f_{G} \delta_{G} p_{K}\right)+\frac{\beta_{0} f_{K}}{\beta_{1} \delta_{K}} \Psi\right)-2 q \Psi\right]^{2} \Phi+\beta_{0}^{2} D^{2}\left(\Phi+\left(\beta_{1} p_{K}+2 f_{K}^{2}\right) \frac{\Psi}{\beta_{1} \delta_{K}^{2}}\right) \Phi \\
- & \beta_{1}\left(\Psi+\frac{\beta_{1} \delta_{K}^{2}}{\beta_{1} p_{K}+2 f_{K}^{2}} \Phi\right) \Psi \cdot\left(\Phi+\frac{\beta_{1} p_{K}+2 f_{K}^{2}}{\beta_{1} \delta_{K}^{2}} \Psi\right) q^{2} \\
+ & {\left[2 \beta_{1} \beta_{0}\left(f_{K} \delta_{K} \psi+f_{G} \delta_{G} p_{K}\right)+2 \beta_{0} \beta_{1} f_{K} \delta_{K} \frac{\Phi}{\beta_{1} p_{K}+2 f_{K}^{2}}\right] \Psi \cdot\left(\Phi+\frac{\beta_{1} p_{K}+2 f_{K}^{2}}{\beta_{1} \delta_{K}^{2}} \Psi\right) q } \\
- & {\left[\beta_{0}^{2}\left(f_{K}^{2} \psi+f_{G}^{2} p_{K}\right)+\beta_{0}^{2} f_{K}^{2} \frac{\Phi}{\beta_{1} p_{K}+2 f_{K}^{2}}\right] \Psi \cdot\left(\Phi+\frac{\beta_{1} p_{K}+2 f_{K}^{2}}{\beta_{1} \delta_{K}^{2}} \Psi\right) } \\
= & C_{2} \cdot q^{2}+C_{1} \cdot q+C_{0}, \text { where }
\end{aligned}
$$




$$
\begin{aligned}
& C_{2}=4 \beta_{1} \Psi^{2} \Phi-\beta_{1}\left[\Psi+\frac{\beta_{1} \delta_{K}^{2}}{\beta_{1} p_{K}+2 f_{K}^{2}} \Phi\right] \Psi \cdot\left[\Phi+\frac{\beta_{1} p_{K}+2 f_{K}^{2}}{\beta_{1} \delta_{K}^{2}} \Psi\right] \\
& =\beta_{1} \Psi \cdot\left[4 \Psi \Phi-\frac{\left[\left(\beta_{1} p_{K}+2 f_{K}^{2}\right) \Psi+\beta_{1} \delta_{K}^{2} \Phi\right]^{2}}{\beta_{1} \delta_{K}^{2}\left(\beta_{1} p_{K}+2 f_{K}^{2}\right)}\right]=\frac{-\beta_{1} \Psi \cdot\left[\left(\beta_{1} p_{K}+2 f_{K}^{2}\right) \Psi-\beta_{1} \delta_{K}^{2} \Phi\right]^{2}}{\beta_{1} \delta_{K}^{2}\left(\beta_{1} p_{K}+2 f_{K}^{2}\right)} \\
& =\frac{-\beta_{1} \Psi Q^{4}}{\left(\beta_{1} p_{K}+2 f_{K}^{2}\right) \beta_{1} \delta_{K}^{2}}<0, \\
& C_{1}=-2 \beta_{1}\left(\beta_{0}\left(f_{K} \delta_{K} \psi+f_{G} \delta_{G} p_{K}\right)+\frac{\beta_{0} f_{K}}{\beta_{1} \delta_{K}} \Psi\right) 2 \Psi \Phi \\
& +\left(2 \beta_{1} \beta_{0}\left(f_{K} \delta_{K} \psi+f_{G} \delta_{G} p_{K}\right)+2 \beta_{0} \beta_{1} f_{K} \delta_{K} \frac{\Phi}{\beta_{1} p_{K}+2 f_{K}^{2}}\right) \Psi \cdot\left(\Phi+\frac{\beta_{1} p_{K}+2 f_{K}^{2}}{\beta_{1} \delta_{K}^{2}} \Psi\right) \\
& =2 \beta_{1} \beta_{0} \Psi \cdot\left[-\left(f_{K} \delta_{K} \psi+f_{G} \delta_{G} p_{K}\right)\left(\Phi-\frac{\beta_{1} p_{K}+2 f_{K}^{2}}{\beta_{1} \delta_{K}^{2}} \Psi\right)-\frac{f_{K} \delta_{K} \Phi}{\beta_{1} \delta_{K}^{2}}\left(\Psi-\frac{\beta_{1} \delta_{K}^{2}}{\beta_{1} p_{K}+2 f_{K}^{2}} \Phi\right)\right] \\
& =\frac{-2 \beta_{0} \beta_{1} \Psi}{\left(\beta_{1} p_{K}+2 f_{K}^{2}\right) \beta_{1} \delta_{K}^{2}}\left[\left(f_{K} \delta_{K} \psi+f_{G} \delta_{G} p_{K}\right)\left(\beta_{1} p_{K}+2 f_{K}^{2}\right)\left[\beta_{1} \delta_{K}^{2} \Phi-\left(\beta_{1} p_{K}+2 f_{K}^{2}\right) \Psi\right]\right. \\
& \left.+f_{K} \delta_{K} \Phi \cdot\left[\left(\beta_{1} p_{K}+2 f_{K}^{2}\right) \Psi-\beta_{1} \delta_{K}^{2} \Phi\right]\right] \\
& =\frac{-2 \beta_{0} \beta_{1} \Psi}{\left(\beta_{1} p_{K}+2 f_{K}^{2}\right) \beta_{1} \delta_{K}^{2}}\left[\beta_{1} \delta_{K}^{2} \Phi-\left(\beta_{1} p_{K}+2 f_{K}^{2}\right) \Psi\right]\left[f_{G} \delta_{G} p_{K}\left(\beta_{1} p_{K}+2 f_{K}^{2}\right)-f_{K} \delta_{K} 2 f_{G}^{2} p_{K}\right] \\
& =\frac{-2 \beta_{0} \beta_{1} \Psi}{\left(\beta_{1} p_{K}+2 f_{K}^{2}\right) \beta_{1} \delta_{K}^{2}}\left(-Q^{2}\right) f_{G} p_{K} Q=\frac{\beta_{1} \Psi 2 \beta_{0} f_{G} p_{K} Q^{3}}{\left(\beta_{1} p_{K}+2 f_{K}^{2}\right) \beta_{1} \delta_{K}^{2}} \text {, and } \\
& C_{0}=\beta_{1}\left(\beta_{0}\left(f_{K} \delta_{K} \psi+f_{G} \delta_{G} p_{K}\right)+\frac{\beta_{0} f_{K}}{\beta_{1} \delta_{K}} \Psi\right)^{2} \Phi+\beta_{0}^{2} D^{2}\left[\Phi+\left(\beta_{1} p_{K}+2 f_{K}^{2}\right) \frac{\Psi}{\beta_{1} \delta_{K}^{2}}\right] \Phi \\
& -\left[\beta_{0}^{2}\left(f_{K}^{2} \psi+f_{G}^{2} p_{K}\right)+\beta_{0}^{2} f_{K}^{2} \frac{\Phi}{\beta_{1} p_{K}+2 f_{K}^{2}}\right] \Psi \cdot\left[\Phi+\frac{\beta_{1} p_{K}+2 f_{K}^{2}}{\beta_{1} \delta_{K}^{2}} \Psi\right]=\frac{\beta_{0}^{2}}{\left(\beta_{1} p_{K}+2 f_{K}^{2}\right) \beta_{1} \delta_{K}^{2}} \cdot \tilde{C}_{0} \text { with } \\
& \tilde{C}_{0}=\beta_{1}\left(f_{K} \delta_{K} \psi+f_{G} \delta_{G} p_{K}\right)^{2} \Phi \beta_{1} \delta_{K}^{2}\left(\beta_{1} p_{K}+2 f_{K}^{2}\right)+\beta_{1}\left(2 f_{K} \delta_{K} \psi+2 f_{G} \delta_{G} p_{K}\right) f_{K} \delta_{K} \Psi \Phi \cdot\left(\beta_{1} p_{K}+2 f_{K}^{2}\right) \\
& +D^{2} \Phi^{2} \beta_{1} \delta_{K}^{2}\left(\beta_{1} p_{K}+2 f_{K}^{2}\right)+\left(f_{K} \delta_{G}-f_{G} \delta_{K}\right)^{2}\left(\beta_{1} p_{K}+2 f_{K}^{2}\right)^{2} \Psi \Phi \\
& -\left(f_{K}^{2} \psi+f_{G}^{2} p_{K}\right) \Psi \Phi \cdot\left(\beta_{1} p_{K}+2 f_{K}^{2}\right) \beta_{1} \delta_{K}^{2}-\left(f_{K}^{2} \psi+f_{G}^{2} p_{K}\right) \Psi^{2} \cdot\left(\beta_{1} p_{K}+2 f_{K}^{2}\right)^{2}-f_{K}^{2} \Phi^{2} \Psi \beta_{1} \delta_{K}^{2} \\
& =\beta_{1}\left(f_{K} \delta_{K} \psi+f_{G} \delta_{G} p_{K}\right)^{2} \Phi \beta_{1} \delta_{K}^{2}\left(\beta_{1} p_{K}+2 f_{K}^{2}\right)+\beta_{1} \psi f_{K}^{2} \delta_{K}^{2} \Psi \Phi \cdot\left(\beta_{1} p_{K}+2 f_{K}^{2}\right) \\
& +D^{2} \Phi^{2} \beta_{1} \delta_{K}^{2}\left(\beta_{1} p_{K}+2 f_{K}^{2}\right)+\left(f_{K}^{2} \delta_{G}^{2}+f_{G}^{2} \delta_{K}^{2}\right)\left(\beta_{1} p_{K}+2 f_{K}^{2}\right)^{2} \Psi \Phi-\left(f_{K}^{2} \psi+f_{G}^{2} p_{K}\right) \Psi^{2} \cdot\left(\beta_{1} p_{K}+2 f_{K}^{2}\right)^{2} \\
& -2 f_{K} \delta_{K} f_{G} \delta_{G} 2 f_{K}^{2} \Psi \Phi \cdot\left(\beta_{1} p_{K}+2 f_{K}^{2}\right)-f_{G}^{2} p_{K} \Psi \Phi \cdot\left(\beta_{1} p_{K}+2 f_{K}^{2}\right) \beta_{1} \delta_{K}^{2}-f_{K}^{2} \Phi^{2} \Psi \beta_{1} \delta_{K}^{2} \\
& =\beta_{1}\left(f_{K} \delta_{K} \psi+f_{G} \delta_{G} p_{K}\right)^{2} \Phi \beta_{1} \delta_{K}^{2}\left(\beta_{1} p_{K}+2 f_{K}^{2}\right)+\beta_{1} \psi f_{K}^{2} \delta_{K}^{2} \Psi \Phi \cdot\left(\beta_{1} p_{K}+2 f_{K}^{2}\right) \\
& +D^{2} \Phi^{2} \beta_{1} \delta_{K}^{2}\left(\beta_{1} p_{K}+2 f_{K}^{2}\right)+f_{K}^{2} \delta_{G}^{2} \beta_{1} p_{K}\left(\beta_{1} p_{K}+2 f_{K}^{2}\right) \Psi \Phi+\left(f_{K}^{2} \delta_{G}^{2}+f_{G}^{2} \delta_{K}^{2}\right) 2 f_{K}^{2}\left(\beta_{1} p_{K}+2 f_{K}^{2}\right) \Psi \Phi \\
& -2 f_{K} \delta_{K} f_{G} \delta_{G} 2 f_{K}^{2} \Psi \Phi \cdot\left(\beta_{1} p_{K}+2 f_{K}^{2}\right)-\left(f_{K}^{2} \psi+f_{G}^{2} p_{K}\right) \Psi^{2} \cdot\left(\beta_{1} p_{K}+2 f_{K}^{2}\right)^{2}-f_{K}^{2} \Phi^{2} \Psi \beta_{1} \delta_{K}^{2} \\
& =\beta_{1}\left(f_{K} \delta_{K} \psi+f_{G} \delta_{G} p_{K}\right)^{2} \Phi \beta_{1} \delta_{K}^{2}\left(\beta_{1} p_{K}+2 f_{K}^{2}\right)-\left(f_{K}^{2} \psi+f_{G}^{2} p_{K}\right) \Psi^{2} \cdot\left(\beta_{1} p_{K}+2 f_{K}^{2}\right)^{2}-f_{K}^{2} \Phi^{2} \Psi \beta_{1} \delta_{K}^{2} \\
& +D^{2} \Phi^{2} \beta_{1} \delta_{K}^{2}\left(\beta_{1} p_{K}+2 f_{K}^{2}\right)+\beta_{1}\left(\psi \delta_{K}^{2}+p_{K} \delta_{G}^{2}\right) f_{K}^{2}\left(\beta_{1} p_{K}+2 f_{K}^{2}\right) \Psi \Phi+2 f_{K}^{2} D^{2}\left(\beta_{1} p_{K}+2 f_{K}^{2}\right) \Psi \Phi \\
& =\beta_{1}\left(f_{K} \delta_{K} \psi+f_{G} \delta_{G} p_{K}\right)^{2} \Phi \beta_{1} \delta_{K}^{2}\left(\beta_{1} p_{K}+2 f_{K}^{2}\right)-\left(f_{K}^{2} \psi+f_{G}^{2} p_{K}\right) \Psi^{2} \cdot\left(\beta_{1} p_{K}+2 f_{K}^{2}\right)^{2}-f_{K}^{2} \Phi^{2} \Psi \beta_{1} \delta_{K}^{2} \\
& +D^{2} \Phi^{2} \beta_{1} \delta_{K}^{2}\left(\beta_{1} p_{K}+2 f_{K}^{2}\right)+\Psi^{2} \cdot\left(\beta_{1} p_{K}+2 f_{K}^{2}\right) f_{K}^{2}\left[\left(\beta_{1} p_{K}+2 f_{K}^{2}\right) \psi+2 f_{G}^{2} p_{K}\right] \\
& =\beta_{1}\left(f_{K} \delta_{K} \psi+f_{G} \delta_{G} p_{K}\right)^{2} \Phi \beta_{1} \delta_{K}^{2}\left(\beta_{1} p_{K}+2 f_{K}^{2}\right) \\
& +\left(f_{K} \delta_{G}-f_{G} \delta_{K}\right)^{2} \Phi^{2} \beta_{1} \delta_{K}^{2}\left(\beta_{1} p_{K}+2 f_{K}^{2}\right)-f_{G}^{2} p_{K} \Psi^{2} \cdot\left(\beta_{1} p_{K}+2 f_{K}^{2}\right) \beta_{1} p_{K}-f_{K}^{2} \Phi^{2} \Psi \beta_{1} \delta_{K}^{2} \\
& =\beta_{1}\left(f_{K} \delta_{K} \psi+f_{G} \delta_{G} p_{K}\right)^{2} \Phi \beta_{1} \delta_{K}^{2}\left(\beta_{1} p_{K}+2 f_{K}^{2}\right)+f_{G}^{2} \delta_{K}^{2} \Phi^{2} \beta_{1} \delta_{K}^{2} \beta_{1} p_{K} \\
& -2 f_{K} \delta_{G} f_{G} \delta_{K} \Phi^{2} \beta_{1} \delta_{K}^{2} \beta_{1} p_{K}-f_{G}^{2} p_{K} \Psi^{2} \cdot\left(\beta_{1} p_{K}+2 f_{K}^{2}\right) \beta_{1} p_{K}-f_{K}^{2} \beta_{1} \delta_{K}^{2} \Phi^{2} \beta_{1} \delta_{K}^{2} \psi \\
& =\beta_{1}\left(f_{K} \delta_{K} \psi+f_{G} \delta_{G} p_{K}\right)^{2} \Phi \beta_{1} \delta_{K}^{2}\left(\beta_{1} p_{K}+2 f_{K}^{2}\right) \\
& +f_{G}^{2} \delta_{K}^{2} \Phi \beta_{1} \delta_{K}^{2} \beta_{1} p_{K}\left[\left(\beta_{1} p_{K}+2 f_{K}^{2}\right) \psi+2 f_{G}^{2} p_{K}\right]-2 f_{K} \delta_{G} f_{G} \delta_{K} \Phi \beta_{1} \delta_{K}^{2} \beta_{1} p_{K}\left[\left(\beta_{1} p_{K}+2 f_{K}^{2}\right) \psi+2 f_{G}^{2} p_{K}\right] \\
& -f_{K}^{2} \beta_{1} \delta_{K}^{2} \Phi \beta_{1} \delta_{K}^{2} \psi\left[\left(\beta_{1} p_{K}+2 f_{K}^{2}\right) \psi+2 f_{G}^{2} p_{K}\right]-f_{G}^{2} p_{K} \Psi^{2} \cdot\left(\beta_{1} p_{K}+2 f_{K}^{2}\right) \beta_{1} p_{K} \\
& =\beta_{1}^{2} \delta_{K}^{2} \Phi \cdot\left[f_{G}^{2} \delta_{G}^{2} p_{K}^{2}\left(\beta_{1} p_{K}+2 f_{K}^{2}\right)-4 f_{K} \delta_{K} f_{G} \delta_{G} p_{K}^{2} f_{G}^{2}+f_{G}^{2} \delta_{K}^{2} p_{K}\left(\beta_{1} p_{K} \psi+2 f_{G}^{2} p_{K}\right)\right] \\
& -f_{G}^{2} p_{K} \Psi^{2} \cdot\left(\beta_{1} p_{K}+2 f_{K}^{2}\right) \beta_{1} p_{K} \\
& =\beta_{1}^{2} \delta_{K}^{2} \Phi f_{G}^{2} p_{K}^{2} \Psi-f_{G}^{2} p_{K} \Psi^{2} \cdot\left(\beta_{1} p_{K}+2 f_{K}^{2}\right) \beta_{1} p_{K}=p_{K}^{2} f_{G}^{2} \beta_{1} \Psi\left[\beta_{1} \delta_{K}^{2} \Phi-\Psi \cdot\left(\beta_{1} p_{K}+2 f_{K}^{2}\right)\right] \\
& =p_{K}^{2} f_{G}^{2} \beta_{1} \Psi \cdot(-1) Q^{2}<0 .
\end{aligned}
$$

Thus,

$$
W_{B}^{M}(q)-W^{M}(q)=\frac{\beta_{1} \Psi \cdot\left[q^{2} \cdot(-1) Q^{4}+q \cdot 2 \beta_{0} f_{G} p_{K} Q^{3}+(-1) \beta_{0}^{2} f_{G}^{2} p_{K}^{2} Q^{2}\right]}{\left(\beta_{1} p_{K}+2 f_{K}^{2}\right) \beta_{1} \delta_{K}^{2} 2 \beta_{1} \Psi \Phi \cdot\left(\Phi+\frac{\left(\beta_{1} p_{K}+2 f_{K}^{2}\right)}{\beta_{1} \delta_{K}^{2}} \Psi\right)} .
$$




\section{C.12 Calculations for Lemma 3b}

$$
\begin{aligned}
\Gamma(q)-\Gamma_{B}(q) & =\frac{\left(\beta_{0} p_{K} f_{G}-q Q\right)^{2}}{4 \Phi \cdot\left(\beta_{1} p_{K}+2 f_{K}^{2}\right)}-\frac{\beta_{1} \delta_{K}^{2} \Psi \cdot\left(\beta_{0} p_{K} f_{G}-q Q\right)^{2}}{\left[\beta_{1} \delta_{K}^{2} \Phi+\left(\beta_{1} p_{K}+2 f_{K}^{2}\right) \Psi\right]^{2}} \\
& =\frac{\left(\beta_{0} p_{K} f_{G}-q Q\right)^{2} \cdot\left[\left[\beta_{1} \delta_{K}^{2} \Phi+\left(\beta_{1} p_{K}+2 f_{K}^{2}\right) \Psi\right]^{2}-4 \Psi \Phi \beta_{1} \delta_{K}^{2}\left(\beta_{1} p_{K}+2 f_{K}^{2}\right)\right]}{4 \Phi \cdot\left(\beta_{1} p_{K}+2 f_{K}^{2}\right)\left[\beta_{1} \delta_{K}^{2} \Phi+\left(\beta_{1} p_{K}+2 f_{K}^{2}\right) \Psi\right]^{2}} \\
& =\frac{\left(\beta_{0} p_{K} f_{G}-q Q\right)^{2} \cdot\left[\beta_{1} \delta_{K}^{2} \Phi-\left(\beta_{1} p_{K}+2 f_{K}^{2}\right) \Psi\right]^{2}}{4 \Phi \cdot\left(\beta_{1} p_{K}+2 f_{K}^{2}\right)\left[\beta_{1} \delta_{K}^{2} \Phi+\left(\beta_{1} p_{K}+2 f_{K}^{2}\right) \Psi\right]^{2}} \\
& =\frac{\left(\beta_{0} p_{K} f_{G}-q Q\right)^{2} \cdot Q^{4}}{4 \Phi \cdot\left(\beta_{1} p_{K}+2 f_{K}^{2}\right)\left[\beta_{1} \delta_{K}^{2} \Phi+\left(\beta_{1} p_{K}+2 f_{K}^{2}\right) \Psi\right]^{2}} .
\end{aligned}
$$

With respect to the gas price, note that $\gamma^{*}<\gamma_{B}^{*} \Leftrightarrow \frac{\Phi}{\beta_{1} p_{K}+2 f_{K}^{2}}<\frac{\Psi}{\beta_{1} \delta_{K}^{2}} \Leftrightarrow \beta_{1} \delta_{K}^{2} \Phi-\left(\beta_{1} p_{K}+2 f_{K}^{2}\right) \Psi<0 \Leftrightarrow-Q^{2}<0$, a true statement. With respect to the demand for gas, note that $G^{*}>G_{B}^{*}\left(\Leftrightarrow \sqrt{G^{*}}>\sqrt{G_{B}^{*}}\right)$ is equivalent to

$$
\begin{aligned}
& \frac{\beta_{0} p_{K} f_{G}-q Q}{2 \Phi}>\frac{2 \beta_{0} \delta_{K}(-D)\left[\Phi+\left(\beta_{1} p_{K}+2 f_{K}^{2}\right) \frac{\Psi}{\beta_{1} \delta_{K}^{2}}\right]+2 Q\left[\beta_{0}\left(f_{K} \delta_{K} \psi+f_{G} \delta_{G} p_{K}\right)+\frac{\beta_{0} f_{K}}{\beta_{1} \delta_{K}} \Psi\right]-4 Q \Psi q}{4 \Psi \cdot\left[\Phi+\left(\beta_{1} p_{K}+2 f_{K}^{2}\right) \frac{\Psi}{\beta_{1} \delta_{K}^{2}}\right]} \\
\Leftrightarrow & \beta_{0} p_{K} f_{G} 4 \Psi \cdot\left[\Phi+\left(\beta_{1} p_{K}+2 f_{K}^{2}\right) \frac{\Psi}{\beta_{1} \delta_{K}^{2}}\right]-2 \beta_{0} \delta_{K}\left(\delta_{K} f_{G}-\delta_{G} f_{K}\right)\left[\Phi+\left(\beta_{1} p_{K}+2 f_{K}^{2}\right) \frac{\Psi}{\beta_{1} \delta_{K}^{2}}\right] 2 \Phi \\
& -2 Q\left[\beta_{0}\left(f_{K} \delta_{K} \psi+f_{G} \delta_{G} p_{K}\right)+\frac{\beta_{0} f_{K}}{\beta_{1} \delta_{K}} \Psi\right] 2 \Phi>q Q 4 \Psi \cdot\left[\Phi+\left(\beta_{1} p_{K}+2 f_{K}^{2}\right) \frac{\Psi}{\beta_{1} \delta_{K}^{2}}\right]-4 Q \Psi q 2 \Phi .
\end{aligned}
$$

The right-hand side of the latter inequality equals $q \cdot 4 Q \Psi \cdot(-1)\left[\Phi-\frac{\beta_{1} p_{K}+2 f_{K}^{2}}{\beta_{1} \delta_{K}^{2}} \Psi\right]=q \cdot 4 Q \Psi \cdot(-1) \frac{-Q^{2}}{\beta_{1} \delta_{K}^{2}}=q \cdot \frac{4 Q^{3} \Psi}{\beta_{1} \delta_{K}^{2}}$, and the left-hand side multiplied by $\frac{\beta_{1} \delta_{K}^{2}}{4 \beta_{0}}$ equals

$$
\begin{aligned}
& \beta_{1} \delta_{K}^{2} p_{K} f_{G} \Psi \Phi+p_{K} f_{G} \Psi^{2} \cdot\left(\beta_{1} p_{K}+2 f_{K}^{2}\right)-\delta_{K}\left(\delta_{K} f_{G}-\delta_{G} f_{K}\right) \Phi^{2} \beta_{1} \delta_{K}^{2} \\
& -\delta_{K}\left(\delta_{K} f_{G}-\delta_{G} f_{K}\right) \Phi \cdot\left(\beta_{1} p_{K}+2 f_{K}^{2}\right) \Psi-Q \Phi \cdot\left(f_{K} \delta_{K} \psi+f_{G} \delta_{G} p_{K}\right) \beta_{1} \delta_{K}^{2}-Q \Phi f_{K} \delta_{K} \Psi \\
= & p_{K} f_{G} \Psi^{2} \cdot\left(\beta_{1} p_{K}+2 f_{K}^{2}\right)-\delta_{K}\left(\delta_{K} f_{G}-\delta_{G} f_{K}\right) \Phi^{2} \beta_{1} \delta_{K}^{2}-Q \Phi \cdot\left(f_{K} \delta_{K} \psi+f_{G} \delta_{G} p_{K}\right) \beta_{1} \delta_{K}^{2} \\
= & p_{K} f_{G} \cdot\left[\Psi^{2} \cdot\left(\beta_{1} p_{K}+2 f_{K}^{2}\right)-\delta_{K}^{2} \Phi \beta_{1} \delta_{K}^{2} \beta_{1} \psi-2 f_{G} \delta_{K}\left(\delta_{K} f_{G}-\delta_{G} f_{K}\right) \Phi \beta_{1} \delta_{K}^{2}\right. \\
& \left.\quad-\beta_{1} \delta_{G}^{2} p_{K} \Phi \beta_{1} \delta_{K}^{2}-2 f_{K} \delta_{G}\left(f_{K} \delta_{G}-f_{G} \delta_{K}\right) \Phi \beta_{1} \delta_{K}^{2}\right] \\
= & p_{K} f_{G} \cdot\left[\Psi^{2} \cdot\left(\beta_{1} p_{K}+2 f_{K}^{2}\right)-\left(\beta_{1}\left(\delta_{K}^{2} \psi+\delta_{G}^{2} p_{K}\right)+2\left(\delta_{K} f_{G}-\delta_{G} f_{K}\right)^{2}\right) \Phi \beta_{1} \delta_{K}^{2}\right] \\
= & p_{K} f_{G} \cdot\left[\Psi^{2} \cdot\left(\beta_{1} p_{K}+2 f_{K}^{2}\right)-\Psi \Phi \beta_{1} \delta_{K}^{2}\right] \\
= & p_{K} f_{G} \Psi \cdot\left(\beta_{1} p_{K}+2 f_{K}^{2}\right)\left[\Psi-\frac{\beta_{1} \delta_{K}^{2}}{\beta_{1} p_{K}+2 f_{K}^{2}} \Phi\right] \\
= & p_{K} f_{G} \Psi Q^{2} .
\end{aligned}
$$

Thus, $G^{*}>G_{B}^{*} \Leftrightarrow \frac{4 \beta_{0}}{\beta_{1} \delta_{K}^{2}} p_{K} f_{G} \Psi Q^{2}>q \cdot \frac{4 Q^{3} \Psi}{\beta_{1} \delta_{K}^{2}}$, which is, if $Q \neq 0$, equivalent to $\beta_{0} p_{K} f_{G}>q \cdot Q$, a true statement (note that $q<\bar{q} \stackrel{\text { def }}{=} \frac{\beta_{0} f_{K} \psi}{R} \leq \frac{\beta_{0} f_{G} p_{K}}{Q}$ if $Q>0$ ).

Finally, $G^{*}=\left(\frac{\beta_{0} p_{K} f_{G}-q Q}{2 \Phi}\right)^{2}>\left(\frac{\beta_{0} p_{K} f_{G}}{2 \Phi}\right)^{2}=G_{A}^{*}$ if $Q<0$, and $G^{*}<G_{A}^{*}$ if $Q>0$.

\section{C.13 Calculations for Lemma 3c}

$$
\begin{aligned}
W_{B}^{C}(q)=W^{C}(q) & \Leftrightarrow \frac{1}{\beta_{1} \Psi \Phi}\left[\beta_{1}\left(\beta_{0}\left(f_{K} \delta_{K} \psi+f_{G} \delta_{G} p_{K}\right)-q \Psi\right)^{2}+\beta_{0}^{2} D^{2} \Phi\right] \\
& =\frac{1}{\beta_{1} \Phi}\left[\beta_{1} \Psi \cdot q^{2}-2 \beta_{1} \beta_{0}\left(f_{K} \delta_{K} \psi+f_{G} \delta_{G} p_{K}\right) \cdot q+\beta_{0}^{2}\left(f_{K}^{2} \psi+f_{G}^{2} p_{K}\right)\right] \\
& \Leftrightarrow \beta_{1}\left(f_{K} \delta_{K} \psi+f_{G} \delta_{G} p_{K}\right)^{2}+D^{2} \Phi=\left(f_{K}^{2} \psi+f_{G}^{2} p_{K}\right) \Psi \\
& \Leftrightarrow \beta_{1}\left(f_{K} \delta_{K} \psi+f_{G} \delta_{G} p_{K}\right)^{2}+\beta_{1} p_{K} \psi D^{2}=\left(f_{K}^{2} \psi+f_{G}^{2} p_{K}\right)\left(\beta_{1} \delta_{G}^{2} p_{K}+\beta_{1} \delta_{K}^{2} \psi\right) \\
& \Leftrightarrow 2 f_{K} \delta_{K} \psi f_{G} \delta_{G} p_{K}+p_{K} \psi D^{2}=f_{K}^{2} \psi \delta_{G}^{2} p_{K}+f_{G}^{2} p_{K} \delta_{K}^{2} \psi \\
& \Leftrightarrow D^{2}=f_{K}^{2} \delta_{G}^{2}+f_{G}^{2} \delta_{K}^{2}-2 f_{K} \delta_{G} f_{G} \delta_{K}\left(=D^{2}\right) .
\end{aligned}
$$

\section{C.14 Calculations for Lemma 4}

If $Q<0$, then $\tilde{q}(Q)<\frac{\beta_{0} f_{K} \psi}{R}$ is equivalent to $A_{2} \cdot \psi^{2}+A_{1} \cdot \psi+A_{0}>0$, where $A_{2}:=2 f_{K}\left(\beta_{1} p_{K}+2 f_{K}^{2}\right) \beta_{1} \delta_{K}^{2}>0$ and $A_{1}:=\beta_{1} \delta_{K} p_{K} f_{G}\left[\beta_{1} \delta_{G} p_{K}+2 f_{K}\left(f_{K} \delta_{G}+f_{G} \delta_{K}\right)\right]>0$.

If $Q>0$, then $\tilde{q}(Q)<\frac{\beta_{0} f_{K} \psi}{R}$ is equivalent to $B_{2} \cdot \psi^{2}+B_{1} \cdot \psi+B_{0}>0$, where $B_{2}:=2 f_{K} \beta_{1} \delta_{K}^{2}\left(\beta_{1} p_{K}+2 f_{K}^{2}\right)>0$. 


\section{References}

[1] M. Grubb and D. Newbery. Pricing Carbon for Electricity Generation: National and International Dimensions. In Delivering a Low Carbon Electricity System, M. Grubb, T. Jamasb, and M. Pollitt (eds.), Cambridge University Press, 2008

[2] C. Hepburn. Regulation by Prices, Quantities, or Both: A Review of Instrument Choice. Oxford Review of Economic Policy 22, 2006

[3] J.P. Montero. Market Power in Pollution Permit Markets. Instituto de Economia, Pontificia Universidad Catolica de Chile, Documentos de Trabajo No. 355, 2009

[4] E.T. Mansur. Prices vs. Quantities: Environmental Regulation and Imperfect Competition. NBER Working Paper No. 13510, 2007

[5] K. Neuhoff. Tackling Carbon: How to Price Carbon for Climate Policy. Climate Strategies Report (www.climatestrategies.org), 2008

[6] D. Newbery. Climate Change Policy and its Effect on Market Power in the Gas Market. Journal of the European Economic Association 6, 2008

[7] R. Newell and W. Pizer. Regulating Stock Externalities under Uncertainty. Journal of Environmental Economics and Management 45, 2003

[8] M. Weitzman. Prices versus Quantities. Review of Economic Studies 41, 1974 


\section{Preprints 2009}

2009/26: Burhop C., The Transfer of Patents in Imperial Germany

2009/25: Burhop C., Lübbers T., The Historical Market for Technology Licenses: Chemicals, Pharmaceuticals, and Electrical Engineering in Imperial Germany

2009/24: Engel C., Competition as a Socially Desirable Dilemma Theory vs. Experimental Evidence

2009/23: Morell A., Glöckner A., Towfigh E., Sticky Rebates: Rollback Rebates Induce Non-Rational Loyalty in Consumers Experimental Evidence

2009/22: Traxler C., Majority Voting and the Welfare Implications of Tax Avoidance

2009/21: Beckenkamp M., Engel C., Glöckner A., Irlenbusch B., Hennig-Schmidt H., Kube S., Kurschilgen M., Morell A., Nicklisch A., Normann H., Towfigh E., Beware of Broken Windows! First Impressions in Public-good Experiments

2009/20: Nikiforakis N., Normann H., Wallace B., Asymmetric Enforcement of Cooperation in a Social Dilemma forthcoming in: Southern Economic Review, In Press.

2009/19: Magen S., Rechtliche und ökonomische Rationalität im Emissionshandelsrecht

2009/18: Broadberry S.N., Burhop C., Real Wages and Labour Productivity in Britain and Germany, 1871-1938: A Unified Approach to the International Comparison of Living Standards

2009/17: Glöckner A., Hodges S.D., Parallel Constraint Satisfaction in Memory-Based Decisions

2009/16: Petersen N., Review Essay: How Rational is International Law? forthcoming in: European Journal of International Law, vol. 20, In Press.

2009/15: Bierbrauer F., On the legitimacy of coercion for the financing of public goods

2009/14: Feri F., Irlenbusch B., Sutter M., Efficiency Gains from Team-Based Coordination - Large-Scale Experimental Evidence

2009/13: Jansen J., On Competition and the Strategic Management of Intellectual Property in Oligopoly

2009/12: Hellwig M., Utilitarian Mechanism Design for an Excludable Public Good published in: Economic Theory, vol. 2009, no. July 14, Berlin/Heidelberg, Springer, 2009.

2009/11: Weinschenk P., Persistence of Monopoly and Research Specialization

2009/10: Horstmann N., Ahlgrimm A., Glöckner A., How Distinct are Intuition and Deliberation? An Eye-Tracking Analysis of Instruction-Induced Decision Modes

2009/09: Lübbers T., Is Cartelisation Profitable? A Case Study of the Rhenish Westphalian Coal Syndicate, 1893-1913

2009/08: Glöckner A., Irlenbusch B., Kube S., Nicklisch A., Normann H., Leading with(out) Sacrifice?

A Public-Goods Experiment with a Super-Additive Player

forthcoming in: Economic Inquiry, In Press.

2009/07: von Weizsäcker C., Asymmetrie der Märkte und Wettbewerbsfreiheit

2009/06: Jansen J., Strategic Information Disclosure and Competition for an Imperfectly Protected Innovation forthcoming in: Journal of Industrial Economics, In Press.

2009/05: Petersen N., Abkehr von der internationalen Gemeinschaft? - Die aktuelle Rechtsprechung des US Supreme Court zur innerstaatlichen Wirkung von völkerrechtlichen Verträgen -

forthcoming in: Völkerrecht im innerstaatlichen Bereich, Vienna, facultas.wuv, In Press. 
2009/04: Rincke J., Traxler C., Deterrence Through Word of Mouth

2009/03: Traxler C., Winter J., Survey Evidence on Conditional Norm Enforcement

2009/02: Herbig B., Glöckner A., Experts and Decision Making: First Steps Towards a Unifying Theory of Decision Making in Novices, Intermediates and Experts

2009/01: Beckenkamp M., Environmental dilemmas revisited: structural consequences from the angle of institutional ergonomics, issue 2009/01

\section{Preprints 2008}

2008/49: Glöckner A., Dickert S., Base-rate Respect by Intuition: Approximating Rational Choices in Base-rate Tasks with Multiple Cues

2008/48: Glöckner A., Moritz S., A Fine-grained Analysis of the Jumping to Conclusions Bias in Schizophrenia: DataGathering, Response Confidence, and Information Integration

2008/47: Hellwig M., A Generalization of the Atkinson-Stiglitz (1976) Theorem on the Undesirability of Nonuniform Excise Taxation

2008/46: Burhop C., The Underpricing of Initial Public Offerings in Imperial Germany, 1870-1896 forthcoming in: German Economic Review, In Press.

2008/45: Hellwig M., A Note on Deaton's Theorem on the Undesirability of Nonuniform Excise Taxation forthcoming in: Economics Letters, In Press.

2008/44: Hellwig M., Zur Problematik staatlicher Beschränkungen der Beteiligung und der Einflussnahme von Investoren bei großen Unternehmen published in: Zeitschrift für das gesamte Handelsrecht und Wirtschaftsrecht, vol. 172, pp. 768-787, 2008.

2008/43: Hellwig M., Systemic Risk in the Financial Sector: An Analysis of the Subprime-Mortgage Financial Crisis published in: De Economist, no. 16.07.2009: Springer US, 2009.

published in: Jelle Zijlstra Lecture, no. 2008/5, Wassenaar, NL, Netherlands Institute for Advanced Study in the Humanities and Social Sciences, Institute of the Royal Netherlands Academy of Arts and Sciences, pp. 100, 2008.

2008/42: Glöckner A., Herbold A., Information Processing in Decisions under Risk: Evidence for Compensatory Strategies based on Automatic Processes

2008/41: Lüdemann J., Magen S., Effizienz statt Gerechtigkeit forthcoming in: Zeitschrift für Rechtsphilosophie, In Press.

2008/40: Engel C., Die Bedeutung der Verhaltensökonomie für das Kartellrecht

2008/39: Bierbrauer F., A unified approach to the revelation of public goods preferences and to optimal income taxation

2008/38: Burhop C., Lübbers T., Incentives and Innovation? R\&D Management in Germany's High-Tech Industries During the Second Industrial Revolution forthcoming in: Explorations in Economic History, In Press.

2008/37: Nicklisch A., Salz T., Reciprocity and status in a virtual field experiment

2008/36: Glöckner A., Engel C., Can We Trust Intuitive Jurors? An Experimental Analysis

2008/35: Lüdemann J., Wettbewerb und Regulierung in der Telekommunikation. Das Telekommunikationsrecht vor den Herausforderungen dynamischer Märkte

published in: Telekommunikation, Energie, Eisenbahn - Welche Regulierung brauchen die Netzwirtschaften?, Tübingen, Mohr Siebeck, pp. 69-99, 2008. 